\title{
MALAYSIAN JOURNAL OF
}

\section{PUBLIC HEALTH MEDICINE}

e-ISSN: 2590-3829

ISSN: 1675-0306

Volume 22 (Supplement 1) 2022

Official Publication of the

MALAYSIAN PUBLIC HEALTH PHYSICIANS' ASSOCIATION 


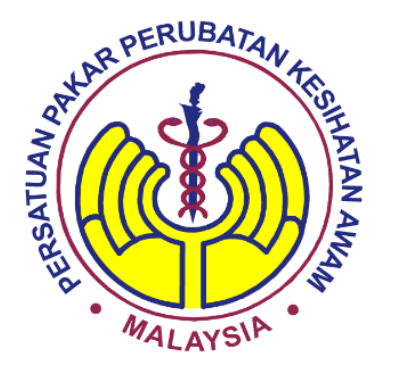

\title{
MJPHM \\ Official Journal of Malaysian Public Health Physicians' Association
}

\section{EDITORIAL BOARD}

\author{
Prof. Dato' Dr. Lokman Hakim Sulaiman \\ Prof. Dr. Maznah Dahlui \\ Prof. Dr. Jamalludin Ab. Rahman \\ Prof. Dr. Saperi Sulong \\ Assoc. Prof. Dr. Retneswari Masilamani \\ Assoc. Prof. Dr. Mariam Mohamad \\ Assist. Prof. Dr. Amrizal Muhd Nur \\ Assist. Prof. Dr. Namaitijiang Maimaiti \\ Dr. Amin Sah Ahmad \\ Dr. Sabrina Che Abd Rahman \\ Dr. Mahanim Md Yusof \\ Dr. Mohd Azahadi Omar
}

Chief Editor

Prof. Dato' Dr. Syed Mohamed Aljunid

Department of Health Policy \& Management, Faculty of Public Health, Kuwait University. International Centre for Casemix and Clinical Coding, Faculty of Medicine, National University of Malaysia.

\section{Deputy Chief Editor}

Prof. Dr. Mohamed Rusli Abdullah

Members:

University Sains Malaysia

Dr. Roslan Johari

Dr. Othman Warijo

Dr. Ghazali Chik

Dr. Tahir Aris
International Medical University (IMU)

University of Malaya (UM)

International Islamic University Malaysia (IIUM)

Universiti Kebangsaan Malaysia (UKM)

Universiti Tunku Abdul Rahman, Malaysia

Universiti Teknologi MARA (UiTM)

Kuwait University

Ankara Yıldırım Beyazıt University

Ministry of Health Malaysia

Ministry of Health Malaysia

Ministry of Health Malaysia

Ministry of Health Malaysia

Ministry of Health Malaysia

Ministry of Health Malaysia

Ministry of Health Malaysia

Ministry of Health Malaysia

\section{Chief Editor}

Malaysian JournalofPublicHealthMedicine(MJPHM)

International Centre for Casemix and Clinical Coding

UniversitiKebangsaan MalaysiaMedicalCentre(UKMMC)

Jalan Yaacob Latif, 56000 Cheras, Kuala Lumpur

Malaysia

\section{ISSN:1675-0306}

TheMalaysian JournalofPublic HealthMedicineispublishedtwiceayear

Copyright reserved @ 2001

Malaysian Public HealthPhysicians' Association

SecretariateAddress:

The Secretariate

International Centre for Casemix and Clinical Coding

UniversitiKebangsaan MalaysiaMedicalCentre(UKMMC)

Jalan Yaacob Latif, 56000 Cheras, Kuala Lumpur

$$
\text { Malaysia }
$$

Tel: 03-91456986/6987/6988 Faks: 03-91456685, Email: editor@mjphm.org 


\section{MJPHM}

Official Journal of Malaysian Public Health Physicians'Association

8 $^{\text {TH }}$ NATIONAL HEALTH SEMINAR
DIGITAL HEALTH: STEPPING INTO THE FUTURE OF MEDICINE
7 $^{\text {TH }}$ OCTOBER 2021
E-CONFERENCE, FAKULTI PERUBATAN DAN SAINS KESIHATAN, USIM, NILAI,
MALAYSIA
EDITORIAL BOARD
Members: $\quad$ Assoc. Prof. Maizatul Azma Binti Masri
Dept of Obstetrics And Gynaecology (O\&G)
FPSK, USIM
Dr. Ruhi Fadzlyana Binti Jailani
Dept of Surgery
FPSK, USIM
Dr. Liyana Azmi
Dept of Microbiology
FPSK, USIM




\section{CONTENTS}

\section{ORAL PRESENTATION}

OP01

OP02

OP03

OP04

OP05

OP06

OP07

OP08

OP09

OP010
Malay Speech-To-Text Proccessing for Forensic Medical Report System Ahmad Taqiyuddin Mohd Anas, Prof Dr Norita Ahmad, Dr Nurul Kharmila Abdullah

Virtual Team-Based Learning (TBL) During Pandemic Noor Fadzilah Zulkifli, Ainoon Othman, Hayati Abd Rahman, Asral Wirda Ahmad Asnawi, Nur Syahrina Rahim, Nurul Kharmila Abdullah, Alina Fauzi

Association Between Level of Knowledge Gained, Confidence, Motivation And Flexibility On Types Of Learning For Bedside Teaching Among Clinical Students In Four Malaysian Medical Schools During COVID-19 Pandemic

Zainal Maaruf, Kamilia; Ngio, Wei Kiat; Balakrishnan, Koustubak; Mohamad Zainal, Nurul Hayati; Mohd Nor Nurul Huda

The Effect of Educational Video Intervention on the Knowledge of Folic Acid Supplementation Among International Islamic University Malaysia (IIUM) Female Undergraduates

Engku Ngah Sayuddin, Engku Nasiha; Rostam, Muhamad Ashraf

Evaluation of Ancestry from Human Cervical Vertebrae: 2-Dimensional Geometric Morphometrics Approach

Mohd Fauad, Muhammad Faiz; Ku Mohd Noor, Ku Mastura; Alias, Aspalilah; Ker Woon Choy; Wei Lin Ng

The Application of Unmanned Aerial Vehicles (UAV) in Geophysical-related Disaster Management: A Scoping Review

Elliza Mansor, Fadzilah Abdullah, Ungku Izmin Farah Ungku Halmie, Khalsom Samsudin, Khairul Nizam Mohd Isa, Aidalina Mahmud

A review on Telemedicine Service Utilization in Malaysia - An Organizational Perspective Fadzilah Abdullah, Aidalina Mahmud, Rosliza Abdul Manaf

Thalamic Probabilistic Connectivity With Cerebral Cortex In Spastic Cerebral Palsy Mukhtar, Siti Fatimah, Mohd Ismail, Zul Izhar, Ahmad, Asma Hayati, Abdullah, Jafri Malin, Simok, Anna Alicia, Sapiai, Nur Asma'

Burnout Among University Students During Pandemic COVID-19: A Scoping Review

Zainuddin, Huzaimi; Abdullah, Natasya

Knowledge, Attitude and Practice of Family Planning and Its Associated Factors Among Adults in Malaysia

Baharom, Nizam; Shamshul Bahri, A. Hisham; Abol Kasim, Balqis; Hishamuddin, Ashiqen; Wan Izudin, Wan Athirah; Mohd Zin, Noorsuwaidah; Mohd Noor, Aidil; Rushdi, Firdaus; Azahari, Effa; Hatta, Hasyimah; Termizi, Siti Hajar; Rosdin, 'Afif; Che Ahmat, Najihah; Abdul Fatah, Sabrina; Basri, Taufeeq; Abdullah, Amalia; Rosli, Asyiqin; Allani Huslan, Asyiqin; Yazid, Nadia; Rahim, Asyraf; Zainoodin, Munir; Saidin, Humaira; Zulkifli, Nur Hanisah; Muji, Nuraisya; Khair, Najmi; Ab Rahman, Amnan; Lukman, Saiful Syahmi; Azzeri, Amirah; Mohamad Yusuff, Aza Sherin; Mohd Rani, Dzulkhairi; Abu Baharin, Fakhri; Mohamad Nor, Nadeeya 'Ayn; T Mohd, Madeehah; A Rahman, Zairina; Jaafar, Mohd Hafiz 
Telemedicine Model of Online Psychotherapy: An Alternative Treatment for Mental Health Wellbeing Nasir, Maasyah; Faizal, Auni; Azlan, Azzam; Rafee, Faris; Azmi, Liyana; Zulkifli, Ayeshah Amri, Nur Syafiqa , Zakaria Rosnani, Mohamad Noraini Development of Malaysian Anthropometric Portal Sarah, Zubir; Siti Zaharah, Abd. Rahman; Hazwan Adli, Hamadan; Nor Shahira, Mohd Salim

Decision Making in Exergames for Rehabilitation Using Artificial Intelligence Method

Zul Hilmi Abdullah; Waidah Ismail; Lailatul Qadri Zakaria; Rimuljo Hendradi

Knowledge, Attitude and Stigma On Tuberculosis And The Associated Factors For Attitude Among Tuberculosis Contacts In Alor Setar, Kedah. Loh, Shin Yee; Zakaria, Rosnani; Mohamad, Noraini

Comparing The Sensitivity of Distortion Product (DP) and Transient-Evoked Emission (OAE) in Healthy Neonates with or Without Vernix in Hospital Universiti Sains Malaysia.

Mohd Saiful Al; Nik Adilah NO; Nik Fariza Husna NH; Mahamad Almyzan A

Lifestyle of Year One Primary School Students with Moderate and Severe Astigmatism in Gua Musang: An Interpretative Phenomenological Analysis (IPA) Nurulain MZ; Syarmilla CS1; Nur Liyana I; Aznor Azwan AA; Ahmad Ziad S; Suraida AR; Muhamad Afzam Shah AR; Abdul Mutalib $O$ Rashid, Mohd Fadzil Nor; Quar, Tian Kar; Maamor, Nashrah; Chong, Foong Yen during COVID-19 Pandemic Hasbullah, Husna; Mohamad Fauzi, Fatin; Shuhaimi, Puteri Alifah; Glam Hadzir, Hafiz; Tengku Mohd, Tengku Amatullah Madeehah; Norazmi, Abu Ubaidah Amir. 
Mandarin Speaking Audiologists: A Preliminary Study

Foong Yen Chong; Cila Umat; Kah Kei Mak; Yan Jin Goh; Badrulzaman Abdul

Hamid; and Bee See Goh Awareness Among Undergraduate University Students: A Systematic Review Mohd Azam, Ummi Amani; Mohd Farezal, Nurul Amarina; Pazudin, 'Imran; Zulkefli, Afiq Zakwan; Che Abd Aziz, Uthman; Mohmad Sanip, Aina Nabilah; Abdul Aziz, Anis; Ahmad Hamizi, Mohamed Aiman!; Khair, Muhammad Basyir; Jasny, Faiq Fauzani; Razali, Khairul Redwan; Mohd Salihuddin, Aina Najeehah; Yazid, Anisah!; Baniamin, Nur Shafiniı; Mohd Shaharudin, Shahidahı; Mukhadzir, Afiqah;

Tajulazhar, Salma; Wan Izani, Izfah: Azzeri, Amirah!; Mohamad Yusuff, Aza Sherin'; Mohd Rani, Dzulkhairi; Mohamad Nor, Nadeeya 'Ayn; Baharom, Nizam; T Mohd, Madeehah; A Rahman, Zairina; Jaafar, Mohd Hafiz; Abu Baharin, Mohamed Fakhri

OP028

OP029

OP030

OP031

OP032

OP034

OP033

Community-Developed Video Clips as An Effective Risk Communication Tool on COVID-19 Preventive Measures by The Indigenous Orang Asli Communities Mohd Dzulkhairi Mohd Rani, Hana Maizuliana Solehan, Nurul Azmawati Mohamed, Nadeeya'Ayn Umaisara Mohamad Nor, Zatul-'Iffah Abu Hasan, Suhaila Sanip

Association Between Blastocystis sp. Infection and Type 2 Diabetes Mellitus in Adults In Malaysia: A Pilot Case Control Study Mohd Shaari, Nurul Saadah; Wan Shahida, Wan Sulaiman; Mohd Rahman, Omar; Nadeeya'Ayn Umaisara; Samseh Abdullah, Noradilah

The use of Virtual OSCE (vOSCEs) in Medical Education: A scoping review Mohd Bakhit, Nour Hanan Daniah; Muhammad Saifullah, Ahmad Anwaar; Jailani, Ruhi Fazlyana

Prevalence of Computer Vision Syndrome and the Associated Factors Among Undergraduate University Students in Malaysia Zainol, Mohamad Akmal Arif; Salahuddin, Nurul Izzati; Md Mokhsen, Siti Nuraisyah; Mohd Sabri, Muhammad Aqil; Wan Zakaria, Wan Nur 'Amirah; Engku Md Azmi, Engku Ahmad llyas; Ahmad Afpandi, Ahmad Haziq; Zulkifli, Muhammad Irfan; Normaly Kamal, Siti Fatimah; Anuar, Aida Syafiqah; Noor Zari, Muhammad Aliff Haziq; Md Haris, Amirul Ameer; Zakaria, Muhammad Zaim; Nor Alkose, Muhammad Naqib Afifuddin; Aman Shah, Normaisarah; Nor Fazli, Nur Izzah Balqis; Abidin, Nur Raihani; Nik Mohamad Faidza, Nik Izzati; Elias, Noor Eza Shazwana; Wan Azemi, Wan Nur Aima Amirah; Azzeri, Amirah; Mohamad Yusuff, Aza Sherin; Mohd Rani, Dzulkhairi; Abu Baharin, Fakhri; Mohamad Nor, Nadeeya 'Ayn; Baharom, Nizam; T Mohd, Madeehah; A Rahman, Zairina; Jaafar, Mohd Hafiz

Knowledge, Attitude and Practice of Health Risks of Video Gaming Towards Eyes, Hearing and Body Posture Among Malaysian Parents with Children Under 18 Years Old

Hassan, Nor Eyzawiah; Muhammad Saifullah, Ahmad Anwaar; Mohd Bakhit, Nour Hanan Daniah; Abu Hasan, Zatul 'Iffah; Abdul Muna'aim, Maimunah; Mohamad Nor, Nadeeya'Ayn Umaisara'

Ethical \& Legal Issues in Remote Medical Consultation â Advocating Regulatory Oversight on Telemedicine in Malaysia Yunus, Aimi Mohd; Katiman, Helwa Husna; Muuti, Muhamad Zaid

Use of 3D based Virtual Learning Resources in Teaching Anatomy: A Scoping Review Ab Rahman, Siti Soraya; Rashid, Zamir 

Children Siti Zuriana MZ

\section{POSTER PRESENTATION}

PP001 Is the Drone Cost Effective? An Economic Evaluation of Drone versus Ambulance for Blood Products Transportation Afiq Hidayat M; Azma RZ; Aniza I; Ismail MS; Rahana AR; Mahdy ZA

PP002

PP004

PP005

PP006

PP007

PP008

PP009

PP011

PP012

PP013
Association Between Walking and Perceived Neighborhood Environments In Seoul, Korea

Choi Mona, Jeon Su Mi

COVID-19 vaccine hesitancy among public in Malaysia: a cross-sectional survey Aws Hashim Ali Al-Kadhim; Faizah Abdul Fatah; Normaliza Ab Malik; Sarah Taha Yousif; Azrul Hafiz Abdul Aziz; Syatirah-Najmi Abdullah; Azlan Jaafar

Child Abuse During The COVID-19 Pandemic Ghani, Syamsul; Yahaya, Yafizah; Ganason, Anu Suria; Syed Mohideen, Fathima Begum

Acute Pancreatitis in Post Total Hip Replacement Azman, Nur Dina; Md Shariff, Mohd Shaffid; Bain, Mohd Asha'ari; Nazarallah, Mohd Hilmi; Norazmi, Abu Ubaidah Amir

Virtual Reality (VR) Locomotion in Increasing Young Adults Physical Activity Tendency Hasiff, Kharmila A

Artificial Intelligence Development in Making Diagnosis and Management Plan Hasiff, Arief $\mathrm{H}$

Running Economy: Secret of Long-distance Running

Zul I; Mohd Noor MT

Usable security in Electronic Health Record System: A review on the Saudi Arabian perspective Samina A. Khan; Syaheerah L. Lutfi

Coding error of Otorhinolaryngology cases in 2020 at one of the University Hospitals in Malaysia according to International Classification of Diseases, Tenth Revision (ICD-10)

Shah Nizamuddin, Muhammad Khairul Asraf; Mohamad Yasim, Mohamad Helmi; Azzeri, Amirah; Sidek, Salawati; Tengku Omar, Tengku Ahmad Shahrizal; Dahlui, Maznah

“Getting Unstuck" in A Disruptive Period of Medical Education: On Uncertainty and Vulnerability of Digital Learning and Moving Forward Through the Lens of Expansive Learning Cycle. 
PP014

PP015

PP016

PP017

PP018

PP019

PP020

PP021

PP022

PP023

PP024

PP025

PP026

Hodgkin Lymphoma with Gastric Invasion: A Case Report

47

Azzahra Azhar; Abu Dzarr Abdullah; Nor Aslina Abd Samat; Nur Asma Sapiai;Faezahtul Arbaeyah Hussain

Dental icon- spreading oral health education through video making during the COVID-19 pandemic

Radzniwan Rashid; Siti Zuriana Mohd Zamzuri; Cheng Lai Choo

The Impact, Challenges, and the Consequences of COVID-19 on NonCommunicable Disease

Zulfa Ghazali; Mohd Radzniwan A. Rashid; Sharifah Najwa Syed Mohamad;

Nuruliza Roslan

Family Planning Uptake among Married Adults in Asia: A Systematic Review Mohamad Yusuff, Aza Sherin; Zainuddin, Huzaimi; Zainal, Adilah; Ahmad Lutfi, Maryam; Abdul Rahman, Shahir; Zaidi, Abrar; Zakaria, Luqman Hakim; Syed Ibrahim, Sharifah Nahidhah; Mokhtar, Mastura; Ariffin, Husna; Ibrahim, Izuddin; Azhar, Khaidir; Samson, Waras; Azhar, Arisa Hanis; Mohd Rani, Dzulkhairi; Abu Baharin, Fakhri; Mohamad Nor, Nadeeya 'Ayn; Baharom, Nizam; T Mohd, Madeehah; A Rahman, Zairina; Jaafar, Mohd Hafiz; Azzeri, Amirah

IR 4.0 - Additive Manufacturing (3D Printing) Emergency Devices Mohd Ifwat Mohd Ghazali, Ahmad Syukran Baharuddin, Muhammad Syafiq Alauddin, Sukor Romat, Muhammad Taufik Mohd Noor

Initiative for Teleconsultation at Klinik Pakar Kesihatan USIM: Embarking on digital health during the COVID-19 Pandemic Syed Mohamad, Sharifah Najwa; A. Rashid, Mohd Radzniwan; Iskandar Mirza, Azrul Azlan

Direct Medical Cost of Stroke and The Cost-Effectiveness of Direct Oral Anticoagulants Among Atrial Fibrillation-Related Stroke Patients in Malaysia Norliana Masbah; Siti Norain Azahar; Saperi Sulong; Wan Asyraf Wan Zaidi; Kamisah Yusof; Norliza Muhammad

Magnetic Resonance Imaging Study as Preferred Method in Diagnosing MorelLavallée Lesion Azman, Nur Dina; Md Shariff, Mohd Shaffid; Bain, Mohd Asha'ari; Nazarallah, Mohd Hilmi; Norazmi, Abu Ubaidah Amir

Dementia Prevention: Food, Exercise and Music Wan Muhammad Najib, Wan Muhammad Nazdmi; Solehan, Hana Maizuliana

Telemedicine during the COVID-19 Pandemic: A Case Series of Our Experience Arief Hamid; Hana Maizuliana Solehan

Occupational Psychological Well-Being Among Emergency Medical Service Ambulance Driver in Hospital Kuala Lumpur Rosdi Zainol $^{1}$, Shuib Rambat ${ }^{2}$, Norazlinda Saad ${ }^{3}$

Crescendo Transient Ischemic Attack' - An Uncommon Presentation of a Very Common Disease: A Case Report on Capsular Warning Syndrome Which Was Reviewed Regularly Via Teleconsultation

Payus, Alvin Oliver; Ibrahim, Azliza; Wan Yahya, Wan Nur Nafisah; Azman Ali, Raymond

An Uncommon Side Effect from Eating Durian Fruit - A Case Report on LifeThreatening Hyperkalaemia After Eating Durian Fruit and Follow Up Via Teleconsultation for Regular Review

Payus, Alvin; Sat Lin, Liew; Azman Ali, Raymond 

Hana Maizuliana Solehan; Nurul Azmawati Mohamed; Mohd Dzulkhairi Mohd Rani; Muslimah Ithnin; Ilina Isahak Badaruddin, Izzatul Aliaa; Md Mansor, Munirah; Wan Shuaib; Wan M Azfar; Ab Rahim, Siti Nadirah; Nasuruddin; Dian Nasriana Towards Management of Medical Emergencies Ahmad Tarmidzi, Nor Azura; Mohamed Ramli, Nalisha; Amran, Norashikin; Norazmi, Husna Najihah; Mohd Arifin, Nur Saniah

Clinical Features and Outcome of ST-Elevation Myocardial Infarction (STEMI) in the Young Population at a Non-PCl Capable Hospital Amran, Norashikin; Low, Wan Chia, Sia, Koon Ket

Estimating Current Active Cases of Covid-19 Post School Re-Opening in Malaysia Using SIR (Susceptible - Infected - Removed) Model Azrul Azim Mohd Yunus; Shahrina Ismail; Muhammad Safwan Ibrahim; Arif Asraf Mohd Yunus Anuar, Balkhis; Abdul Hamid, Nazefah Begum; Abd Rahman, Hayati

Digitalisation of birth registration and health systems in Malaysia: Boon or bane for the hard-to-reach and marginalised? Mohamed Razali, Rodziana; Duraisingam, T.J.; T Mohd, TAM

Shaari, Nurhafizah

Correlation of Biomarkers Changes After Conventional Rehabilitation Therapy in Ischemic and Hemorrhagic Stroke Patients in Hospital Universiti Sains Malaysia Khiati Hadjer; Hafiz, Muhammad Hanafi; Muzaimi, Mustapha; Kassim, Nur Karyatee Abu 'Ubaidah Amir 
Malaysian Journal of Public Health Medicine, Vol. 22 (Suppl (1) 2022

$8^{\text {th }}$ National Health Seminar 2021, Digital health: Stepping into the Future of Medicine, 7th October 2021

PP044

PP046

PP047

PP048

PP049

PP050

PP051

PP052

PP055

PP057

PP058

PP059

PP061

PP063

Clinical Engagement Through Telemedicine During COVID-19 Pandemic \& Going Beyond Muhammad, Amalin Kamilah; Mohamad Nazarallah, Mohamad Hilmi

Sudden Sensorineural Hearing Loss in COVID-19: Our Experience Lye Meng Hon; Carren Teh Sui Lin; Nik Adilah Binti Nik Othman

Contributing Factors of LMA Usage in Pre-Hospital Care Among Assistant Medical Officers Abdul Ghafar, Muhammad Ariffin; Naganathan, Ratneswaran; Mamat, Wahidi; Zainol, Zohori

Effectiveness of the Teleaudiology Approach for Hearing Assessment and Intervention: A Comprehensive Review Rashid, Mohd Fadzil Nor; Hussain, Mohd Khary; Mahmud, Halimah; Ahmad, Rozazipah; Mansor, Suzana; Mohd Zainudin, Nur Syazana

Physical and Mental Health Impacts of Watching Mukbang in Young Adult Zulkefli, Siti Hajjar; T Mohd, Tengku Amatullah Madeehah

Hand Grip Strength and its Associated Factors Among E-sports And Video Gaming Players: A Cross-sectional Study Protocol Mohd Kamil, Muhammad Kashani; Muhammad Yazid, Muhammad Ashraff; Ismail, Khairil Idham; Salim, Ahmad Munawwar Helmi; Amir Norazmi, Abu Ubaidah

LGBT: An Uprising Worldwide Phenomenon - A Systematic Review on Academics and Psychological Impacts among Children with LGBT Parents Ahmad Affandi, Arni Farisha; Mohamad Nor, Nadeeya 'Ayn Umaisara

The Impact of COVID-19 to Healthcare Service Utilization in Malaysia: A Scoping Review Izani, Izlyn; Jaafar, Hafiz'; Mohd Nor, M

A Case of Salivary Gland Choristoma Presenting with Ear Discharge in a Child with Canal Stenosis

Mohd Saiful Al; Nik Adilah NO; Farhana MM; Sharifah Emilia.TS; Mohd Khairi MD

COVID-19 Pandemic: Increasing in Gadget and Internet Addiction Among Children Rahim, Huda; Mokhtar, Azlina

Susceptibility to COVID-19 And IIIness Severity in Elderly Patients: A Comparison with Paediatric Age Group Romizhi, Aliah Fatin; Nor Aripin, Khairun Nain

Neglected Foreign Body Oesophagus Complicated by Tracheo-Oesophageal Fistula Mohamad Najib bin Salleh; Azliana Aziz; Hashimah Ismail; Wan Zainira Wan Zai; Mohd Nizam Md Hashim; Irfan Mohamad

A Systematic Review: Consideration of The Psychological Impact on Male Partners in The Management of Early Pregnancy Loss Karali Hassan Fadhil; Poh, Dawn Siaw Celine; Phong, Yen Wen; Soorianarayanan, Parveena; Ting Jason Siong; Dr Zaigham Muhammad Talha

The Association of Dietary Intake and Acne Vulgaris: A Naturopathic Perspective Wan Anuar, Wan Nur Adlina; Mohamad Nor, Nadeeya 'Ayn Umaisara; Abu Hasan, Zatul-'Iffah 
PP065

PP066

PP067

PP068

PP069

PP070

PP071

PP072

PP073

PP074

PP075

PP077

PP078
Giant Botryoid Fibroepithelial Polyp as an Unusual Cause of Obstructive Uropathy in a 6-Year-Old Girl Azzahra Azhar; Mohamad Ikram Ilias; Mohamed Ashraf Mohamed Daud; Ahmad Hadif Zaidin Samsudin; Nur Asyilla Che Jalil

Oral Contrast for Magnetic Resonance Enterography: Comparison Between Pineapple Juice and Different Mannitol Concentration Zulkifle, Ahmad Farid; Abdul Hamid, Hamzaini

Critical Value Notification in a Chemical Pathology Laboratory of Tertiary Teaching Hospital: An Analysis Wan Norlina, Wan Azman; Aniza, Mohammed Jelani; Julia, Omar; Noorazliyana, Shafii; Nor Azlin Azraini, Che Soh; Tuan Salwani, Tuan Ismail; Nur Karyatee, Kassim; Hanim Afzan, Ibrahim; Ahamd Ezam, Zainan; Wan Mohd Faizal, Wan Azman

Kelantan Multi-Disaster Risk Assessment and Best Practice During Pandemic Abdul Ghafar, Muhammad Ariffin; Abdul Jalal, Aishah Sabrina: Yahya, Nor Yazjehan; Shaikh Mohd Salleh, Siti Nurliyana; Razak, Khamarrul Azahari

Sinonasal Malignant Melanocytic Melanoma as a Rare Cause of Epistaxis Mohamad, Adam; Saravannan, Pavitra; Yusuf, Suhaimi; Abdul Wahab, Azwan Halim; Mohd Rajet, Khairul Azhar; Mohamad, Irfan, Ramli; Ramiza Ramza

Study of Hematocrit Level in Obstructive Sleep Apnea Syndrome Jamaluddin, Jamal Sazly; Mohamad, Hazama; Ramli, Ramiza Ramza

Knowledge, Attitude and Practice Towards Sharp Injuries Prevention Among Nurses

Seman, Nordianna; Albert, Jennifer

Parathyroid Adenoma in a Patient with Renal Cell Carcinoma: A Rare Association Kassim,Nur Karyatee; Yahya, Maya Mazuwin;Tuan Ismail, Tuan Salwani; Thien Jun, Jun; Mohamad, Irfan

Undetectable HbA1c Level in a Diabetic Patient with Leukaemia Wan Nik, Wan Nor Fazila Hafizan; Tuan Ismail, Tuan Salwani; Kassim, Nur Karyatee

Potential Protective Effects of Tualang Honey on Chronic Combinatory Stressedrat Testis

Mohd Amin, Mohamad Syabil Ikhwan; Mohd Yusof, Nurul Aiman; Norman, Haziq Hazman; Asari, Mohd Asnizam

Survey on Exercise-Related Leg Pain (ERLP) among University Recreational Runners

Latir Aliff; Bakhtiar Jamily Ahmed Ali Bashir

The Knowledge, Attitude, and Practice of Physical Activity Among Community Dwelling Stroke Survivors in Klang Valley, Malaysia

Ong Ke Yee; Lian Sin Jie; Haniz Naqib bin Hazaidi: Nor Azlin Mohd Nordin

Blended Clinical Learning for Undergraduate Paediatric Rotation Post-Pandemic: the USIM Experience

Rusli, Nur Sabrina; Mohammad Khuzaini, Aliyyah; Abdul Halim, Halimah; Maimun Aqsha, Nur Syahirah; Suhaidi, Siti Sarah Aishah 

and Practices of Public Nordin, Norshahrizan; Ishak, Najwahanim; Che Razak, Razli; Shaharuddin, Muhd Ikram Fikrie; Ramdzan, Abdul Rahman; Saadon, Mohd Saiful Izwaan

A Critical Incident Technique (CIT) Measurement for Analysis of Complaints and Compliments among Hospital Patients: A Case Study in Northern Region, Malaysia Nordin, Norshahrizan; Anuar, Siti Athirah; Che Razak, Razli; Shaharuddin, Muhd Ikram Fikrie; Saadon, Mohd Saiful Izwaan

PP084

PP085

PP086

PP087

PP088

PP089

PP090

PP091

PP092

PP093

Postpartum Body Weight and Body Mass Index Changes Between Exclusive Breastfeeding and Non-Exclusive Breastfeeding Mothers of 6-Months Postpartum Balqis Sofeyya Binti Mohd Zawahid, Farhanah Ahmad Shuhaimi, Naleena Devi Muniandy

Cortical Motor Potency and Muscle Plasticity Interplay Following Noninvasive Brain Stimulation in Ischaemic Stroke Patients Mah Hussain, Mohd Izzat Hakimi; Hanafi, Muhammad Hafiz; Mustapha, Muzaimi; Kassim, Nur Karyatee; Simok, Anna Alicia

Protecting our Elderly: Senior Care during COVID-19 crisis Aziz, Amira Aqila; A. Rashid, Mohd Radzniwan; Mohd Manzor, Nur Fariha, Addnan, Faizul Helmi; Roslan, Nuruliza

Attitudes, Beliefs and Perceived Barriers in Assessment of Sexual Health among Nursing Students

Fatimah Sham, Amira Safiah Saihuddin, Frannelya Francis, Siti Munirah Abdul Wahab, Nordianna Seman

Royal Free Hospital Nutrition Priotizing Tools (RFH-NPT): Predictor of Malnutrion Risk Among Chronic Liver Disease Patients in Malaysia Norashimah Rajab; Syahrul Bariah Abdul Hamid; Khairil Anuar Md Isa; Aishah Hanum Said

Online Distance Learning for Clinical Nutrition Practice Courses Mazuin Kamarul Zaman; Noor Izati Roslan; Nurfarah Hanani Amir Hamzah; Muhammad Hakim Abdul Jalil; Siti Fasihah Abdul Razak; Siti Nor Fatihah Ismail

Why are Children not affected by Severe or Fatal COVID-19? A Preliminary, Digital Systematic Review on Protective Immuno-Pathophysiological Mechanisms in the Paediatric Population Nor Aripin; Khairun Nain Bin, Sani; Anuar, A.Rashid; Mohd Radzniwan, Addnan; Faizul Helmi, Mansur; Fadlul Azim Fauzi, Baharom; Nizam, Anuar; Khairullah

Ocular Surface Squamous Neoplasia: A Snowy Complication Hassan, Faisal, Yaakub, Azhany, Zakariah, Sakinah, Saizaul, Zubaidah

The Effect of Fetal Movement Counting on Maternal Anxiety and Experiences in a Private Hospital

Ahmad, Aini Lim Y, Y

Audiology Students' Attitude Towards Online Simulated Clinical Training Quar, Tian Kar; Ishak, Wan Syafira; Yusoff, Nur Izzati; Yusoff, Wan Nur Hanim; Abdul Aziz, Nadiah; Jamaludin, Mohd Khairil Azahar 

Immune Thrombocytopenia (ITP): a case report. Lye Meng Hon; Intan Kartika Kamarudin; Norhafiza Mat Lazim Rozi, Ramiza lylia; Yusoff, Siti Nur Ainina Mohd; Syed Mohideen, Fathima Begum; Yahaya, Yafizah; Ganason, Anu Suria; Mohd Noor, Muhammad Tauffik Ismail, Anis Afikah; Hanafi, Muhammad Hafiz; Kassim, Nur Karyatee ${ }^{3}$ Ibrahim, Al Hafiz

Development and Initial Validation of The Questionnaire Assessing the Perception and Practice of Workplace Violence Prevention at Healthcare Facilities Mohd Nizam Mohamad Yazid; Nik Rosmawati Nik Husain; Aziah Daud

Estimated Medical Costs of the Top-five Cardiovascular Cases in 2020 at one the University Hospitals in Malaysia Mohamad Yasim, Mohamad Helmi; Shah Nizamuddin, Muhammad Khairul Asraf; Abu Hasan, Nurhasnira; Azzeri, Amirah; Zainal Abidin, Imran; Dahlui, Maznah

Instructors' Satisfaction towards Online Distant Learning for Clinical Nutrition Practice Courses.

Mazuin Kamarul Zaman; Syahrul Bariah Abdul Hamid; Ahmad labal Azam to the Solution

Amin, Nurul Syafiqah; Mohd Saiful Sega, Intan Ku Khalilah; Tajudin, Najiah; Jamal Abdul Nasir, Nurul Aisyah

Performing the Proper Nasopharyngeal Swab in the COVID-19 pandemic Aman Shah, Normaisarah; Salahuddin, Nor Azirah; Md Arepen, Siti Asmat; Hassan, Nor Eyzawiah for intraocular lens (IOL)power calculation in cataract surgery S, Sharan; $Y$ L, Lee; A,Zalifa Zakiah; M,Azlina;R, Jamaliah Fatimah; Mohammad Kazmin, Nur Ezzaty; Sia, Koon Ket Mellitus 

Induced Cytotoxicity in Mice BALB/C Jayanti, Neti Eka; Mohd Said, Rozzana; I'tishom, Reny; Utami, Rinda Aulia; Sumiati, Raudah, Siti; Achmad, Arisandy; Jaiddin, Kasim Nurhas; Sulfandi, Usman, Rezky Amaliah Treat? Mohammad Kazmin, Nur Ezzaty; W.J.Tiau; Nordin, Fatimah Zanirah; Solehan, Hana Maizuliana; Tan, Wei Ming of Invasive GBS Infection in Adult Mohammad Kazmin, Nur Ezzaty; Kori, Najma; Solehan, Hana Maizuliana; Nordin, Fatimah Zanirah; Tan, Wei Ming using Integrative Bioinformatics Analysis Che Ismail Che Lah; Nik Yusnoraini Yusof; Norhafiza Mat Lazim; Sarina Sulong; Zilfalil Alwi; Baharudin Abdullah

Promoting Ethical Use of social media Among Healthcare Professionals in the Digital Era Katiman, Helwa Husna; Yunus, Aimi Mohd; Muuti, Muhamad Zaid

PP115 The Uprising Homebirth Trend Yusoff, Nurul Afifah; Masri, Maizatul Azma

Development and Utilisation of Breastfeeding Apps to Increase Breastfeeding Mohammad Khuzaini, Aliyyah Zulkifli, Nursyazwani Aimi; Omar, Mohd Rahman Literature Review Izuddin, Ummu Farhah; Abdul Muna'aim, Maimunah Lockdowns Ismail, Suraya; Abdullah Suhaimi, Afiqah; Azmi, Liyana of Acute Stroke in A Non-Epilepsy Centre, A Case Report Solehan, Hana Maizuliana; Tan, Wei Ming; Zanirah, Fatimah, Mohd Kazmin, Nur Ezzaty; Samad, Sarah Zulaikha; Sharil, Nor Safiqah; A Bakar, Mohd Firdaus; Norazinizah Ahmad Miswan Evidence from Malaysia Azzeri, Amirah; Jaafar, Hafiz; Mohamad Nor, Nadeeya 'Ayn; Abdullah, Amalia; Rosli, Asyiqin; Allani Huslan, Asyiqin; Yazid, Nadia; Rahim, Asyraf; Zainoodin, Munir; Saidin, Humaira; Zulkifli, Nur Hanisah; Muji, Nuraisya; Khair, Najmi; Ab Rahman, Amnan; Lukman, Saiful Syahmi; Basri, Taufeeq ; S.Bahri, A.Hisham ; Abol Kasim, Balqis Wan Izudin, Wan Athirah; Mohd Zin, Noorsuwaidah; Mohd Noor, Aidil; Rushdi, Firdaus; Azahari, Effa 
PP126

PP127

PP128

PP129

PP130

PP131

PP132

PP133

PP135

PP137

Breastfeeding vs Artificial feeding - The Cognitive Development Zamri, Jannatunnajwa; Masri, Maizatul Azma

The Prevalence of Refractive Error Among Primary School in Gombak District, 140 Selangor Prior To COVID-19 Pandemic. Abdul Muna'aim, Maimunah; Zainol, Norlelawati; Mokhtar, Azlina; Khairudin, Muhammad Najmi'; Ghani, Nor Diyana

Evidence for the Anthelmintic Effect of Date Palm Fruit: From Ethno-Religious Manuscript Wisdom to Digital Imaging Najm, Omaima Adil; Abdul-Rahman, Asma; Addnan, Faizul Helmi; Elkadi, Mohamed Adel, Mohd-Manzor, Nur Fariha; Abdullah, Wan Omar; Mansur, Fadlul Azim Fauzi

Nursing Students' Knowledge and Attitude toward Sexual Health Concerns Fatimah Sham, Norhayati Abdul Rahman, Siti Munirah Abdul Wahab, Frannelya Francis, Norhafizatul Akma Shohor

Cavities with intracavitary mass in COVID-19: a Diagnostic Dilemma Tan, Cynthia K. Y.; Rohaizat, Fathin Izni Izzati; Sharma, Deepali; Ab Rahman, Siti Soraya

The Role of Virtually Adaptive Simulated Patients in Undergraduates Medical Training: A Scoping Review.

Zainal, Nur Zaireena; M. Thamrin, Norashikin; Surya Rangkuti, Harry; Masri, Maizatul Azma; Mohd Aris, Muhammad Shamsir

Highly Active Relapsing Remitting Multiple Sclerosis with Optic Neuritis and Transverse Myelitis; Fingolimod as an Induction Therapy?

Zanirah, Fatimah; Tan, Hui Jan; Solehan, Hana Maizuliana; Mohammad Kazmin, Nur Ezzaty; Tan, Wei Ming, Samad, Sarah Zulaikha; Sharil, Nor Safiqah; A Bakar, Mohd Firdaus

Left Ventricular Clots with Dilated Cardiomyopathy in COVID-19 Infection; An Underreported Complication of COVID-19 Myocarditis? Zanirah, Fatimah; Solehan, Hana Maizuliana; Mohammad Kazmin, Nur Ezzaty; Tan, Wei Ming; Samad, Sarah Zulaikha; Sharil, Nor Safiqah; A Bakar, Mohd Firdaus

Macklin Phenomenon as a Complication of COVID-19 Samad, Sarah Zulaikha; Sia, Koon Ket; Solehan, Hana Maizuliana; Tan, Wei Ming; Mohammad Kazmin, Nur Ezzaty; Zanirah, Fatimah

Digital Health Literacy and Online Information Seeking during COVID-19: Are We Digitally Literate? Khebir, Norliyana A; Mohd R, Nur SA; Mohmad L, Nur FA; Nik MA, Nik HAJ; Azzeri, Amirah; Azmi, Liyana

Advancement of Telemedicine in Southeast Asia During COVID-19 Pandemic: A Review Fauzi, Shyafiqah; Azmi, Liyana

Gene Expression as a Precision Medicine Tools in Cervical Cancer: A Review Abdul Hamid, Nazefah; Roslan, Nuruliza; Zainal, Nur Zaireena2 Abd Rahman, Hayati 


\section{ORAL PRESENTATION}

\section{ID10 OP01}

\section{Malay Speech-To-Text Processing for Forensic Medical Report System}

$\underline{\text { Ahmad Taqiyuddin Mohd Anas }}{ }^{1}$, Prof Dr Norita Ahmad², Dr Nurul Kharmila Abdullah ${ }^{3}$

${ }^{1}$ Faculty of Science and Technology, University of Science Islam Malaysia, Malaysia

${ }^{2}$ Faculty of Medicine and Health Sciences, University of Science Islam Malaysia, Malaysia

Speech to text refers to the conversion of spoken words into written texts. Problem arose when medical forensic autopsy transcriber needs to jot down post-mortem notes in the surgery room which is time consuming. A Malay speech to text solution is proposed for forensic medical dictation system addressing these requirements. The objectives of this project were to develop Malay speech-to-text conversion technique, to develop an automated functional transcript prototype for Malay speech to text forensic dictation report and evaluate performance of the automated Malay speech-to-text functional transcript prototype for forensic autopsy. Qualitative analysis by interviewing medical expert for the project requirement in the implementation of Waterfall Model from Software Development Life Cycle (SDLC). The working prototype for Malay speech to text specialized for dual language (Malay and English) forensic medical report with accuracy of $87 \%$ and error rate of $13 \%$. The purpose of this study which is to develop Malay speech-to-text conversion technique is achieved. However, some limitations arise that can be improved in future works. 


\section{ORAL PRESENTATION}

\section{ID11 OP02}

\section{Virtual Team-Based Learning (TBL) During Pandemic}

Noor Fadzilah Zulkifli ${ }^{1}$, Ainoon Othman ${ }^{1}$, Hayati Abd Rahman!, Asral Wirda Ahmad Asnawi ${ }^{1}$, Nur Syahrina Rahim, Nurul Kharmila Abdullah ${ }^{1}$, Alina Fauzi ${ }^{1}$

${ }^{1}$ Fakulti Perubatan dan Sains Kesihatan, Universiti Sains Islam Malaysia (USIM)

Team-based learning (TBL) has been used widely to facilitate active learning. It is student-centred, involving students' interaction with course materials and peers. With the recent pandemic and movement control order, TBL sessions had to be implemented virtually. The aim of this study is to identify the student's perception on virtual TBL and the impact on the student's learning. TBL modules were designed for pathology classes among the 3rd year medical students in Universiti Sains Islam Malaysia (USIM). Course materials were provided virtually in the form of lectures, videos and lecture notes. Students attempt weekly individual readiness assurance test (iRATs) using MOODLE followed by team readiness assurance test (tRATs) in multiple channels in Microsoft Teams platform. Upon submission of tRATs, all students resume to a main meeting channel for discussion and application exercises, facilitated by the lecturer. At the end of the course, a feedback form was distributed to the students $(n=72)$. Data was analysed using SPSS version 23.0. Results showed that the team members contribute their ideas to complete the task $(93.1 \%)$. They can maintain the group dynamics (87.5\%) and majority feels that the team displayed a positive attitude during the sessions (91.4\%). They functioned as an effective team virtually $(79.2 \%)$ and managed to understand the concepts and achieved the learning objectives (97.2\%). They appreciate team-learning in the virtual TBL $(87.5 \%)$ and found it meaningful and effective (77.8\%). Although the physical TBL was thought to be more effective, majority of the students $(62.5 \%)$ preferred to have TBL virtually compared to physical TBL. Conclusion: Virtual TBL is engaging and effective as a student centred learning activity and was appreciated as a worthwhile learning experience. Considering this evidence, virtual TBL is encouraged especially during the pandemic period. 


\title{
ORAL PRESENTATION
}

\section{ID13 OP03}

\section{Association Between Level of Knowledge Gained, Confidence, Motivation and Flexibility on Types of Learning for Bedside Teaching Among Clinical Students in Four Malaysian Medical Schools During COVID-19 Pandemic}

\author{
Zainal Maaruf, Kamilia ${ }^{1}$; Ngio, Wei Kiat ${ }^{1}$; Balakrishnan, Koustubak ${ }^{1}$; Mohamad Zainal, Nurul Hayati² ${ }^{2}$ Mohd \\ Nor, Nurul Huda². \\ ${ }^{1}$ Faculty of Medicine and Health Sciences, Universiti Putra Malaysia, Serdang, Selangor, Malaysia \\ ${ }^{2}$ Department of Anatomy, Faculty of Medicine and Health Sciences, Universiti Putra Malaysia, Serdang, \\ Selangor, Malaysia
}

With the rise of the COVID-19 pandemic, higher institutions are forced to change the method of delivery for bedside teaching sessions from face-to-face to online learning. However, online learning was found not effective in delivering practical knowledge and skills to students. This study aims was to determine the association between level of knowledge gained, confidence, motivation, and flexibility on types of learning for bedside teaching sessions among clinical students in four Malaysian medical schools during COVID-19 pandemic. A cross-sectional study involving medical students from Universiti Putra Malaysia (UPM), Universiti Sains Islam Malaysia (USIM), Universiti Islam Antarabangsa (UIA) and Universiti Sains Malaysia (USM) were conducted from 1st March 2021 until 6th June 2021. An online questionnaire was distributed, and it consisted of 5 sections which cover sociodemographic information, level of knowledge gained, confidence, motivation, and flexibility from bedside teaching session. The data was analysed by using SPSS software program to determine the chi square test results. There is an association between the level of knowledge gained, level of confidence, level of motivation and level of flexibility with the type of learning (online or face-to-face) during bedside teaching sessions. Results revealed that students gained a higher level of knowledge (84.9\%), higher level of confidence in physical examination (93.3\%), higher motivation (82.2\%) and higher flexibility $(64.1 \%)$ during face-to-face bedside teaching sessions compared to online learning. Most of the medical students in four Malaysian medical schools prefer face-to-face learning compared to online learning for bedside teaching sessions. 


\section{ORAL PRESENTATION}

\section{ID14 OP04}

\section{The Effect of Educational Video Intervention on the Knowledge of Folic Acid Supplementation Among International Islamic University Malaysia (IIUM) Female Undergraduates}

Engku Ngah Sayuddin, Engku Nasiha'; Rostam, Muhamad Ashraf ${ }^{1}$

${ }^{1}$ Department of Nutrition Sciences, Kulliyyah of Allied Health Sciences, International Islamic University Malaysia, Kuantan, Pahang, Malaysia

Knowledge regarding folic acid supplementation remains unsatisfactory among women of child-bearing age in Malaysia. Despite official recommendations to begin supplementation prior to conception to prevent Neural Tube Defects (NTD), many women remain unaware until the first antenatal check-up, losing the crucial time window of prevention. Educational videos could be a promising tool to address this issue. The objective of this pilot study was to assess the effectiveness of an educational video in increasing knowledge regarding folic acid supplementation among IIUM female undergraduates. This pilot intervention study was carried out in the IIUM Kuantan campus. IIUM female undergraduates $(n=31)$ were recruited through convenient sampling. Inclusion criteria included being actively enrolled and of childbearing age. Participants were excluded if they were under the age of 18 , married, pregnant, or have had experience of pregnancy. The educational video developed for the study was content validated by 6 experts $(\mathrm{CVI}>0.83)$. Participants were first requested to complete a questionnaire to assess baseline knowledge, after which they were shown the educational video. Participants were then requested to complete the same questionnaire one week later to observe changes in knowledge scores post-intervention. Data was analysed in SPSS Version 12.0.1 using descriptive analysis, ANOVA, and Paired t-test $(\mathrm{p}<0.05)$. Mean baseline knowledge was found to be poor. Participants' main source of knowledge was found to be the internet, and no significant difference was found in baseline knowledge between different study backgrounds. The mean knowledge scores significantly increased from 6.61 preintervention to 12.77 post-intervention $(p<0.001)$. The educational video was found to be effective in increasing knowledge regarding folic acid supplementation among IIUM female undergraduates. Our findings point towards the need for intervention among this demography and greater investment in public education regarding folic acid supplementation through online video platforms. 


\section{ORAL PRESENTATION}

\section{ID16 OP05}

\section{Evaluation of Ancestry from Human Cervical Vertebrae: 2- Dimensional Geometric Morphometrics Approach}

Mohd Fauad, Muhammad Faiz $;$; Ku Mohd Noor, Ku Mastura ${ }^{1 *}$; Alias, Aspalilah" ${ }^{2 *}$; Ker Woon Choy ${ }^{3 *}$; Wei Lin Ng ${ }^{4}$

${ }^{1}$ Department of Medical Sciences 1, Faculty of Medicine and Health Sciences, Universiti Sains Islam Malaysia, Nilai, Negeri Sembilan, Malaysia

${ }^{2}$ Department of Basic Sciences and Oral Biology, Faculty of Dentistry, Universiti Sains Islam Malaysia, Kuala Lumpur, Malaysia

${ }^{3}$ Department of Anatomy, Faculty of Medicine, Universiti Teknologi MARA, Sungai Buloh Campus, Selangor, Malaysia

${ }^{4}$ Department of Biomedical Imaging, University Malaya Medical Center, Kuala Lumpur, Malaysia

Ancestry determination is one of the challenging tasks faced by forensic anthropologists due to the complex relationship between skeletal morphology and social constructs. The methods used for ancestral determination incorporates the correlation between skeletal morphology, geographical origin, and environmental factors. Geometric morphometric method utilizes qualitative and quantitative description of an object and is widely recognized for high reliability and reproducibility. The aim of this study was to determine the variation of cervical vertebrae among ancestry of adult Malaysian population using 2-dimensional (2D) geometric morphometric method. Lateral cervical radiographs of 432 subjects comprising of three main ancestries of Malaysian which are Malay, Chinese and Indian of known individuals were retrieved retrospectively. The subjects comprised of both sex of adult Malaysian populations aged from 20 to 60 years old. Fifty-three landmarks were applied on the digitalized radiographs by TPSDig2 (Version 2.31) software. Geometric morphometric analysis was performed by MorphoJ software. The first ten principal components (PC) exhibited $69.78 \%$ variation in cervical vertebrae shapes. Procrustes ANOVA indicated that shape was significantly different among the ancestries $(p<0.001)$. Canonical variate analysis revealed significant differences of both mahalanobis and procrustes distances among ancestries with substantial individuals overlap within groups. Discriminant function analysis (DFA) showed $57.8 \%$ of classification accuracy. This study has found significant cervical vertebrae shape differences among ancestry of Malaysian population with a comparable classification accuracy percentage with other bones. To the best of our knowledge, this is the first study to report such findings in Malaysian population. 


\section{ORAL PRESENTATION}

\section{ID23 OP06}

\section{The Application of Unmanned Aerial Vehicles (UAV) in Geophysical- related Disaster Management: A Scoping Review}

Elliza Mansor ${ }^{1}$, Fadzilah Abdullah ${ }^{1}$, Ungku Izmin Farah Ungku Halmie ${ }^{1}$, Khalsom Samsudin ${ }^{1}$, Khairul Nizam Mohd Isa ${ }^{1}$, Aidalina Mahmud ${ }^{2}$

${ }^{1}$ Department of Community Health, Faculty of Medicine and Health Sciences, Universiti Putra Malaysia, Serdang, Selangor, Malaysia

${ }^{2}$ Senior lecturer, Department of Community Health, Faculty of Medicine and Health Sciences, Universiti Putra Malaysia, Serdang, Selangor, Malaysia

Unmanned aerial vehicle (UAV) or drone is one of the technology advancements that could be used in various activities in disaster management phases: mitigation, preparedness, response, and recovery phase. This scoping review aimed to determine the role of UAV or drones' applications in various geophysical-related disaster management cycles and issues and challenges related to its application. Scopus, Ebscohost/Medline, Springer Link, Science Direct, and IEE databases were searched for articles on UAVs application in geophysical-related disaster. Twentyseven articles were included in this review. UAVs applications were thematic based on the core disaster management phases. For the mitigation phase, UAVs are used as tools for risk assessment and hazard analysis, mapping and monitor the landslide vulnerability, and assist in the reconstruction plan to build resilient infrastructure. Meanwhile, UAVs are used to forecast and plan for the evacuation warning and process in the preparedness phase. UAVs use is widely used in the disaster response phase to assist the transportation of materials to disaster areas, search, and rescue activities, enable network and communication services, and data collection and organisation for mapping affected areas. For the recovery phase, UAVs assess the severity and mapping of the disaster area, monitors the recovery process, assists the disposal of disaster waste, and plans and reconstructs building post-disaster events. UAVs have promising and important roles in all levels of management of various geophysical-related disasters. The application of UAV in geophysical-related disasters is mainly for search and rescue, communication, data collection, monitoring and mapping, and risk assessment and analysis. The issues and challenges of using UAVs in these disasters are lack of awareness and cooperation at the community level, required high cost and level of technologies, risks for information breach, and UAVs capability to be operated in challenging conditions. 


\title{
ORAL PRESENTATION
}

\section{ID24 OP07}

\section{A review on Telemedicine Service Utilization in Malaysia - An Organizational Perspective}

Fadzilah Abdullah' ${ }^{1}$, Aidalina Mahmud ${ }^{2}$ Rosliza Abdul Manaf ${ }^{2}$

\author{
${ }^{1}$ Doctor in Public Health Candidate, Department of Community Health, Faculty of Medicine and Health \\ Sciences, Universiti Putra Malaysia, Serdang, Selangor, Malaysia \\ 2 Senior lecturer, Department of Community Health, Faculty of Medicine and Health Sciences, Universiti Putra \\ Malaysia, Serdang, Selangor, Malaysia
}

Significant interdisciplinary commitment and continuing participation from all key stakeholders are required for the eventual success of telemedicine technology adoption and dissemination. To enhance the demand for telemedicine, both service management and referral guidelines in relation to telemedicine practice should be improved. Additional efforts should be made to remove impediments linked to organizational physical attributes to enable the efficient deployment of telemedicine technology. The aim of this paper is to give information on telehealth governance and policy, as well as to examine the obstacles and significant concerns that may have an influence on the usage of telemedicine in Malaysia, particularly in terms of governance and policy. There will be two sections to this paper. The first section will provide an overview of Malaysia's telehealth governance and policy. The second section will explain the constraints and significant concerns surrounding the adoption of telemedicine. The findings indicated that due to various implementation challenges that have yet to be resolved, progress in the usage of telemedicine has been modest and uneven. Certain organizational, technological, legal, and regulatory barriers have been discovered that hinder telemedicine from being effectively utilized to the point where it can deliver the entire spectrum of medical treatments. Establishing sound governance mechanisms is recommended to help facilitate effective and transparent collaboration, necessary to successfully implement telemedicine services in Malaysia. 


\section{ORAL PRESENTATION}

\section{ID27 OP08}

\section{Thalamic Probabilistic Connectivity with Cerebral Cortex In Spastic Cerebral Palsy}

Mukhtar, Siti Fatimah ${ }^{1}$, Mohd Ismail, Zul Izhar ${ }^{1}$, Ahmad, Asma Hayati ${ }^{2}$, Abdullah, Jafri Malin ${ }^{3}$, Simok, Anna Alicia ${ }^{1}$, Sapiai, Nur Asma' ${ }^{4}$

${ }^{1}$ Department of Anatomy, School of Medical Sciences, Health Campus, Universiti Sains Malaysia, 16150 Kubang Kerian, Kota Bharu, Kelantan.

${ }^{2}$ Department of Physiology, School of Medical Sciences, Health Campus, Universiti Sains Malaysia, 16150 Kubang Kerian, Kota Bharu, Kelantan.

${ }^{3}$ Department of Neuroscience, School of Medical Sciences, Health Campus, Universiti Sains Malaysia, 16150 Kubang Kerian, Kota Bharu, Kelantan.

${ }^{4}$ Department of Radiology, School of Medical Sciences, Health Campus, Universiti Sains Malaysia, 16150 Kubang Kerian, Kota Bharu, Kelantan.

Motor impairment is the main disabling impact of spastic cerebral palsy (SCP). Whether the pathways between the thalamus to motor cortex in spastic cerebral palsy are disrupted remains unclear and the study on its pattern of connectivity is sparse. This retrospective case-control study aims to investigate the probabilistic connectivity between the thalamus and three motor areas of cerebral cortex in spastic cerebral palsy patients. Probabilistic tractography was performed on secondary MRI data of ten healthy control and ten non-aged-matched SCP patients in Hospital Universiti Sains Malaysia. Thalamus was set as the seed region while the three areas of motor cortex, particularly primary motor cortex, premotor cortex and supplementary motor area were the target regions. Connection probability index, which is the indication of white matter integrity, was measured between the thalamus to each motor cortex. The thalamus was further parcellated according to its connection with specific motor cortex. Qualitative analysis was performed. It has been found that the pattern of thalamo-cortical connectivity in cerebral palsy was variable according to the patient's clinical presentation. Thalamic parcellation in control showed that the thalamic cluster with positive connection to primary motor cortex was associated with the lateral group nuclei, which contain the thalamic motor nuclei. A striking feature of thalamic parcellation in SCP was the presence of cluster with positive connection to supplementary motor area. The findings suggest that the SCP brain network was unique according to the clinical manifestation. There was also evidence of neuroplasticity as a compensatory mechanism for the motor deficit in spastic cerebral palsy. 


\section{ORAL PRESENTATION}

\section{ID33 OP09}

\section{Burnout Among University Students During Pandemic COVID-19: A Scoping Review}

$\underline{\text { Zainuddin, Huzaimi }}^{1}{ }^{\text {; }}$ Abdullah, Natasya $^{1}$

${ }^{1}$ Faculty of Medicine and Health Sciences, Universiti Sains Islam Malaysia (USIM), Negeri Sembilan, Malaysia

The COVID-19 pandemic has led to changes in human life which affect the physical, mental, and social wellbeing. Within the education sector, the shift to remote learning has put certain degree of burden and pressure towards the students who need to cope with the new methods and having to adjust to the new norm of living. The impact of such changes may lead to burnout which could manifest as depression, anxiety, and stress. This study aims to examine the prevalence, factors, and determinants of burnout among university students during the COVID-19 pandemic. We also look at the coping mechanisms adapted by the students to overcome the problem. We adopted a scoping study design based on Arksey Methodological Framework 2015. Electronic databases of MEDLINE, EBSCOhost and BMC and PROQUEST were used to identify relevant studies using pre-determine keyword, as well as cross-references searched. All findings were summarised thematically. A total of 10 articles met the inclusion criteria, all of which consist of observational studies. The prevalence of burnout was taken as those who developed depression, anxiety, stress which range from $11 \%$ to $82 \%$. Higher prevalence was seen in female, those who live with family and with underlying health issues. In addition, many of the students significantly have suicidal thoughts, poor anger management and personality dysfunction $(p<0.05)$. Several factors were identified as the possible causes of burnout including disruption of the academic activities, fear of contracting the disease as well as lifestyle changes. Self-resilience and good social support were positively associated with reduction of stress levels among the students. Burnout among university students is a serious issue during the pandemic. Early recognition is crucial to prevent detrimental consequences and to provide proper intervention. Longitudinal studies would be useful to look for the mental health outcome of among those who developed burnout. 


\section{ORAL PRESENTATION}

\section{ID42 OP10}

\section{Knowledge, Attitude and Practice of Family Planning and Its Associated Factors Among Adults in Malaysia}

Baharom, Nizam; Shamshul Bahri, A. Hisham ${ }^{1}$; Abol Kasim, Balqis' ${ }^{1}$ Hishamuddin, Ashiqen ${ }^{1}$; Wan Izudin, Wan Athirah $^{1}$; Mohd Zin, Noorsuwaidah ${ }^{1}$; Mohd Noor, Aidili ${ }^{1}$; Rushdi, Firdaus ${ }^{1}$; Azahari, Effa ${ }^{1}$; Hatta, Hasyimah ${ }^{1}$; Termizi, Siti Hajar' ${ }^{1}$; Rosdin, 'Afif ${ }^{1}$; Che Ahmat, Najihah ${ }^{1}$; Abdul Fatah, Sabrina ${ }^{1}$; Basri, Taufeeq ${ }^{1}$; Abdullah, Amalia ${ }^{1}$; Rosli, Asyiqin ${ }^{1}$; Allani Huslan, Asyiqin ${ }^{1}$; Yazid, Nadia ${ }^{1}$; Rahim, Asyraf ${ }^{1}$; Zainoodin, Munir ${ }^{1}$; Saidin, Humaira; Zulkifli, Nur Hanisah; Muji, Nuraisya; Khair, Najmi; Ab Rahman, Amnan; Lukman, Saiful Syahmi; Azzeri, Amirah ${ }^{1}$; Mohamad Yusuff, Aza Sherin ${ }^{1}$; Mohd Rani, Dzulkhairi ${ }^{1}$; Abu Baharin, Fakhri ${ }^{1}$; Mohamad Nor, Nadeeya 'Ayn'1; T Mohd, Madeehah'1; A Rahman, Zairina' ; Jaafar, Mohd Hafiz'

${ }^{1}$ Public Health Unit, Department of Primary Health Care, Faculty of Medicine and Health Sciences, Universiti Sains Islam Malaysia

The Malaysian Government first introduced Family Planning Policy in 1969. Despite the promotion and campaign on contraceptive use, the utilisation of family planning services in Malaysia is still low. This paper aims to describe the factors associated with knowledge, attitude, and practice of family planning among adults in Malaysia. A cross-sectional study was conducted from 19th April to 6th July 2021 among Malaysian adults. Convenience sampling method was used, and data was collected using an online questionnaire. Overall, 402 adults were included in this study. 331 $(82.3 \%)$ of the respondents were females and $71(17.7 \%)$ were males. Their mean (SD) age was $41.8(9.73) .389(96.8 \%)$ of them were married and $13(3.2 \%)$ were either widowed or divorced. The mean (SD) knowledge and practice score of family planning among the respondents were 14.5 (4.55) out of 24 and 18.18 (5.83) out of 30 respectively while the median (IQR) of attitude score was, 41 (9) out of 50. Factors that were significantly associated with knowledge were age, gender, income, educational level, geographical location, spousal communication, and accessibility ( $p$-value $<0.05)$. For attitude, the significant factors include age, gender, ethnicity, religion, educational level, number of children, spousal communication, cost, and accessibility ( $\mathrm{p}$-value $<0.05$ ). Finally, the significant factors that were associated with practice were age, spousal communication, and accessibility ( $p$-value $<0.05)$. Findings from this study could be used to improve on the education and awareness campaign of family planning services. It could also facilitate decision making in family planning policy in Malaysia. 


\section{ORAL PRESENTATION}

\section{ID60 OP11}

\section{G-Empowered Healthcare - Towards a 'Smart' Medical System}

$\underline{\text { Azlan, Azzam }^{1}}$; Nasir, Maasyah ${ }^{1}$; Faizal, Auni ${ }^{1}$; Rafee, Faris ${ }^{1}$; Azmi, Liyana ${ }^{1}$.

${ }^{1}$ Faculty of Medicine \& Health Sciences, Universiti Sains Islam Malaysia, Nilai, Negeri Sembilan, Malaysia

$5 G$ is 5th generation wireless mobile network, designed to provide high-speed connectivity, zero-latency rates, and greater data capacity. Smart healthcare is an integration of patients and doctors onto a common platform for digital intelligent health monitoring. Implementation of 5G in Artificial Intelligence, Internet of things (loT), wearable devices, software, and application will promote new possibilities in the healthcare landscape including imaging, diagnostic, data analytics, consultation, and treatment. We aim to review the role and potential of $5 \mathrm{G}$ wireless connections in building a smart healthcare system. Some of key requirements for 5G implementation, challenges of $5 \mathrm{G}$ application, and ideas to overcome those limitations will be discussed. We used search strategy using some of the keywords such as " $5 G$ ", "digital", "smart", and "health care system". The literature review was done using Google Scholar, Medline, journals, and articles on relevant topics. Thirty studies from our review demonstrated that $5 \mathrm{G}$ technology was able to form a smart network by connecting billions of devices, applications, and sensors in the healthcare system. Improved data transfer and extended capacity enabled realtime connection between doctors and patients. $5 \mathrm{G}$ provides minimal cost, less timeconsuming, faster medical consultation, limitless connection, and reduces incapability of health care visits. Physicians were able to obtain other specialists' opinions for better treatment quality. 5G network requires some advancement like massive MIMO (Multiple-Input Multiple-Output), small cells, ultra-dense network, and cognitive radio technology. However, several challenges appeared including public acceptance of $5 \mathrm{G}$, cost of infrastructure building, data security, and interoperability issues. These can be handled by promoting campaigns, proper budgets, professionals hiring, and scientific research. Studies had showed that 5G network is beneficial to society by connecting the healthcare system and community. Future expansion of the healthcare system should include the implementation of $5 \mathrm{G}$ as this would improve healthcare delivery. 


\section{ORAL PRESENTATION}

\section{ID62 OP12}

\section{Telemedicine Model of Online Psychotherapy: An Alternative Treatment for Mental Health Wellbeing}

Nasir, Maasyah ${ }^{1}$; Faizal, Auni ${ }^{1}$; Azlan, Azzam ${ }^{1}$; Rafee, Faris ${ }^{1}$; Azmi, Liyana ${ }^{1}$; Zulkifli, Ayeshah ${ }^{1}$

${ }^{1}$ Faculty of Medicine \& Health Sciences, Islamic Science University of Malaysia, Nilai, Negeri Sembilan, Malaysia

Virtual consultation is a form of telemedicine and conducting virtual clinic via live streaming is nothing new however, the widespread pandemic has given telemedicine a significant boost, particularly in the field of group therapy. Significant evidence shows that online psychotherapy is supportive behavioral therapy which by engaging the model likely to diminish user 'attrition'. Psychotherapy generally called talktherapy, uses psychological and verbal techniques, to overcome their problems in desired ways, in the process of treating psychological disorders. Online psychotherapy will be a more authentic form of psychotherapy that integrates therapy with the actual relationship experiences of an individual. This study aims to review the effectiveness of online psychotherapy during the COVID-19 pandemic and as a prospective future treatment to improve cognitive - behavioral problems amongst Malaysians. We used search strategy using some of the keywords such as "online psychotherapy", "digital healthcare" and "telemedicine". Literature review was done using Google Scholar, Medline and PUBMED electronic databases, as well as books, journals, and articles on relevant and associated topics. Some of the top arguments in favor of online psychotherapy were easier access to psychotherapy, service availability and flexibility, enhanced communication and benefits related to client characteristics. Convenience, fulfillment, acknowledgement, acceptance, and increased demand also favors online psychotherapy. Economical preferences clearly show the effectiveness of online psychotherapy. However, there are some arguments against engagement in online psychotherapy such as confidentiality issues may lead to legal consequences, record security issues and protection as well medication prescription especially dose modification. Specialist need for special training and research gaps also have been argued against online psychotherapy. Several studies stated the possibilities to establish a stable and positive therapeutic relationship virtually with respect and trust. This review may help the health professionals in choosing whether to engage in online psychotherapy. 


\title{
ORAL PRESENTATION
}

\section{ID64 OP13}

\section{Incorporating a Robot into an Anxiety Therapy Management System}

\author{
$\underline{\text { Azizi Ab Aziz }}{ }^{1}$
}

${ }^{1}$ Relational Machines Group, Human-Centred Computing Lab, School of Computing, Universiti Utara Malaysia

Robo-based therapy is a framework of human-robotic interactions to reconstruct a person's negative experiences mediated by technological tools. This paper focuses on developing a robot-based therapy called CAKNA for persons with anxiety traits and states. Individual differences in personality traits have been associated with both resilience to stress and stress-induced anxiety. It is essential to acknowledge that nearly one-half of those diagnosed with depression are also diagnosed with anxiety states and traits. The main objective of this project is to design robot-based psychotherapy to support individuals with anxiety traits and states. To achieve this, we develop computational intelligence (agent-based and machine learning) approaches to evaluate individuals and automatically integrate this into our robot. The human experiment was conducted to evaluate the acceptance and usability of robot-based therapy among individuals with mild to moderate anxiety traits. Twenty-one participants (mean=21.5, SD=1.6) were recruited based on a voluntary approach in this project. After the interaction process was completed, participants filled out 4-point forced-choice Likert-type response scales to evaluate their current state. All interactions with the system/ robot were recorded (with consent) for review by experts. The deep breathing exercises were used as a therapy tool to combat anxiety states among respondents. The results have shown that mean (SD) S-Anxiety Form Y scores are (pre-test) 63.3(5.09) and (post-test) 36.9(4.2) with a pvalue of 0.045 . The qualitative results from interview sessions with respondents have shown positive acceptance towards the implementation of CAKNA in reducing their anxiety. This paper explores the use of a robot-based anxiety management system that is responsive to user interaction. The experimental results from both quantitative and qualitative evaluation have demonstrated the potential implementation of such a robotic system through a human experiment study in which people with anxiety state interacted and followed the therapy session. 


\title{
ORAL PRESENTATION
}

\section{ID65 OP14}

\section{Stigma and Depression among Tuberculosis Patient in Kedah, Malaysia}

\author{
Amri, Nur Syafiqa ${ }^{1}$, Zakaria Rosnani ${ }^{1}$, Mohamad Noraini ${ }^{2}$. \\ ${ }^{1}$ Family Medicine Department, School of Medical Sciences, Universiti Sains Malaysia, Health Campus, \\ 16150, Kubang Kerian, Kelantan, Malaysia. \\ ${ }^{2}$ School of Dental Sciences, Universiti Sains Malaysia, Health Campus, 16150, Kubang Kerian, Kelantan, \\ Malaysia.
}

Tuberculosis $(\mathrm{Tb})$ related stigma is one of the important barriers in global Tb control and prevention programme. Tb stigma often cause psychological distress among patients which can impede their disease coping ability, affect treatment adherence, and reduce quality of life. More importantly, stigma may also lead to depression and these conditions frequently coexist, exerting negative impact on the management of Tb. To determine the level of stigma among Tb patients and the factors associated with it as well as to ascertain the relationship between level of stigma and depression. This is a cross sectional study using convenient proportionate sampling method. We recruited $200 \mathrm{~Tb}$ patients from three tertiary government hospitals in Kedah. Case report form, a Malay version of 12 item Tb stigma scale (TSS) and Beck Depression Inventory were used for data collection. Data were then analyzed using simple and multiple linear regression. The mean score of tuberculosis stigma from patient perspective was $13.3(6.87)$. The TSS scale has a minimum score of 0 while maximum score is 36 . Younger age patient, currently married, higher education, unemployment, and HIV co-infection were significantly associated with higher stigma experienced. In addition, there was a moderate, positive correlation between stigma and depression which was statistically significant $(r=0.345, p<0.001)$. The mean score of tuberculosis related stigma was not as high as we initially postulated. Nevertheless, higher level of stigma was found to be statistically correlated with higher depression level. Thus, intervention to reduce stigma among tuberculosis patients can be helpful as it may reduce the depression level. 


\section{ORAL PRESENTATION}

\section{ID67 OP15}

\section{Development of Malaysian Anthropometric Portal}

Mohd Nur Ikhwan, Shafiee ${ }^{1}$; Raemy, Md Zein ${ }^{1}$; Mohd Esa, Baruji ${ }^{1}$; Evelyn, Tan ${ }^{1}$; Siti Nur Sarah, Zubir ${ }^{1}$; Siti Zaharah, Abd. Rahman ${ }^{1}$; Hazwan Adli, Hamadan'; Nor Shahira, Mohd Salim ${ }^{1}$

${ }^{1}$ Consultation Research \& Development Department, National Institute of Occupational Safety and Health (NIOSH), Bandar Baru Bangi, Selangor, Malaysia.

An anthropometric valuation of a group of people is critical in developing ergonomic products, medical devices, and workstations for all populations to reduce the risk of musculoskeletal injuries. At the same time, the failure to strategize a proper data management technique of the thousands of collected anthropometric information can be problematic. The study's objectives are to, i) collect 55 body dimensions and five static muscle strengths, and ii) develop an online anthropometric portal. The equipment used for measurement was an anthropometer, customized anthropometric grid, and dynamometers. The data collection took place nationwide, including Peninsular Malaysia, Sabah, and Sarawak. An online web-based portal was developed by using the same model as the existing database in other countries. 1851 males and 1698 females between 18-69-year-old participated in the study. Descriptive analysis revealed that males were superior to females in all dimensions except for standing hip breadth, sitting hip breadth, thigh clearance, hip circumference, and chest depth. A web-based online portal was developed to ensure the collected data is systematically stored and managed. The online portal will serve as the living database. All anthropometric information from other researchers can be added and retrieved without any cost. The online portal will provide important information for various applications such as forensic medicine, cosmetic surgery, sports equipment, industrial design, clothing design, ergonomics, and architecture. 


\title{
ORAL PRESENTATION
}

\section{ID71 OP16}

\section{Classification Exergames for Rehabilitation Using Artificial Intelligence Method}

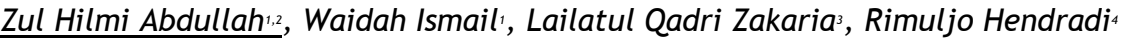

'Faculty of Science and Technology, Universiti Sains Islam Malaysia, Nilai, Negeri Sembilan, Malaysia. 2Faculty of Information Technology, INTI International University, Nilai, Negeri Sembilan, Malaysia Information System Study Program, Faculty Science and Technology, Universitas Airlangga, Indonesia Kampus C, Surabaya, Indonesia.

${ }^{4}$ Center For Artificial Intelligence Technology, Faculty of Information Science and Technology

Rehabilitation process aims to enable disable patients to maintain optimal function to achieve return-to-work (RTW) process. Exercise games is one of the virtual reality treatments (VRT) to help patient with disability in improving the movement of their limbs. VRT is a modern interactive application that integrates computer software with hardware devices such as screen, computer, sensor or Kinect to create an interactive virtual environment for playing exergames (i.e., games with exercises). Artificial Intelligence (Al) is the simulation of human intelligence processess by machines. In this project, using machine learning (ML)as a subset of Al that involves training to make useful prediction using data. The method used are Decision Tree and Neural Network. The study aims to perform comparison between two methods: Decision Tree and Neural Network in classification. The study was carried out in the rehabilitation center of Melaka. The Medical Interactive Recovery Assistance (MIRA) platform generates 26 attributes consisting of patient data file including patients' personal information such as first and last names, patient ID and birthdate. The information related to the games played such as the sessionID, name of the game, movement ID, movement name was recorded. Before patients play the MIRA, patients were required to select the settings such as sides used (left or right), duration, difficulty, tolerance, minimum and maximum range. The experiment was divided into $70 \%$ as training data and $30 \%$ as actual data. The results show decision tree performs classification better than Neural Network. Decision tree is good to use for clean data and the supervised learning. Neural Network will perform bias in the node when required the distribution of the data. In the decision making, decision tree perform better than neural network. In the decision trees, hons clause decided in determine the leaf. The authors would like to acknowledge the supporters of this project which is a part by Fundamental Research Grant Scheme FRGS, Ministry of Higher Education with Application ID 225085-266898 and reference code FRGS/1/2018/ICT02/USIM/02/1, University Sains Islam Malaysia (USIM) 


\title{
ORAL PRESENTATION
}

\section{ID72 OP17}

\section{Knowledge, Attitude and Stigma on Tuberculosis And The Associated Factors For Attitude Among Tuberculosis Contacts In Alor Setar, Kedah}

\author{
Loh, Shin Yee "; Zakaria, Rosnani '; Mohamad, Noraini.
}

\author{
'Family Medicine Department, School of Medical Sciences, Universiti Sains Malaysia, Health Campus, 16150 \\ Kubang Kerian, Kelantan, Malaysia. \\ 2School of Dental Sciences, Universiti Sains Malaysia, Health Campus, 16150 Kubang Kerian, Kelantan, \\ Malaysia.
}

Tuberculosis (TB) is a worldwide public health crisis and it infects millions of people each year worldwide. Malaysia was categorised as an intermediate TB-burden country. TB contact is referring to any individual who was exposed to a person with TB disease. TB contacts are at higher risk of acquiring TB infection and they are candidates for active and latent TB infection screening and eventual treatment. Thus, the World Health Organization (WHO) considers investigation of TB contacts as an essential programme to control TB. The aim of the study was to explore the level of knowledge, attitude and stigma on TB and the associated factors for attitude score among TB contacts in Kedah, Malaysia. A cross-sectional study was performed among TB contacts using a validated structured knowledge, attitude, and stigma on TB questionnaire. There were 338 respondents selected via convenience sampling from the 5 health clinics in Kedah, Malaysia. The data was analysed using simple and multiple linear regression. The mean (SD) for knowledge, attitude, and stigma scores for participants are $73.48 \%(17.06), 82.95 \%$ (10.33), and $66.99 \%$ (16.72) respectively. Income, knowledge score and stigma score were found statistically significantly associated with attitude score $(p<0.001)$. Our study highlighted that the TB contacts generally have a satisfactory knowledge, positive attitude, and high stigma on TB. The significant associated factors for better attitude towards TB were higher income, better knowledge, and higher stigma on TB. Implementation of TB intervention programmes particularly addressing on health education about knowledge on TB are required. More studies should be conducted across TB contacts as they are the main reservoir for new TB cases. 


\title{
ORAL PRESENTATION
}

\section{ID79 OP18}

\section{Comparing The Sensitivity of Distortion Product (DP) and Transient- Evoked (TE) Otoacoustic Emission (OAE) in Healthy Neonates With or Without Vernix in Hospital Universiti Sains Malaysia.}

\author{
Mohd Saiful Al', Nik Adilah NO', Nik Fariza Husna NH', Mahamad Almyzan A 2 \\ Department of Otorhinolaryngology-Head \& Neck Surgery, School of Medical Sciences, Universiti Sains \\ Malaysia Health Campus, 16150 Kubang Kerian, Kelantan, Malaysia. \\ Department of Audiology, School of Medical Sciences, Universiti Sains Malaysia Health Campus, 16150 Kubang \\ Kerian, Kelantan, Malaysia
}

Hearing screening evaluation is very important to every neonate with or without risk factors. Early intervention and intensive rehabilitation will increase their quality of life, thus decreasing hearing problems' negative effects. The study compared the sensitivity of distortion product otoacoustic emission (DPOAE) and transient-evoked otoacoustic emission (TEOAE) in healthy neonates with or without vernix occlusion in Hospital Universiti Sains Malaysia. Our study aimed to compare which OAE is more reliable to use in daily neonatal hearing screening. The cross-sectional study was conducted in Hospital Universiti Sains Malaysia (HUSM), Kubang Kerian, Kelantan. The participants were randomly selected among healthy neonates that meet the stated inclusion and exclusion criteria. Each participant will undergo hearing assessments that include otoscopy and otoacoustic emission (DPOAE and TEOAE). The inclusion criteria are neonates without any known risk factors for hearing loss and all healthy neonates born in HUSM aged less than 48 hours, irrespective of with or without the presence of vernix. The exclusion criteria are neonates admitted to Neonatal ICU or Neonates with pinna and ear canal deformity. There was a significant association between DPOAE and Vernix (5.08 (1), $\mathrm{p}=0.024)$ and TPOAE and Vernix (8.23 (1), $p=0.004)$. There was a significant association between DPOAE and TPOAE (63.37 (1), $p<0.001)$. The sensitivity between the DPOAE and TPOAE test was $83.6 \%$, and its specificity was $82.5 \%$, acceptable. Meanwhile, Cohen's $\mathrm{k}$ test was a moderate agreement between the two OAE judgements and it was statistically significant $(\mathrm{K}=0.578, \mathrm{p}<0.001)$. Both $\mathrm{OAE}$ are affected by the presence of the vernix or debris in the ear canal. TEOAE showed a better pass in comparison to DPOAE in vernix ears. However, there is still a need to further study by using automated brainstem response (AABR) as a standard tool to compare DPOAE and TPOAE. 


\title{
ORAL PRESENTATION
}

\section{ID81 OP19}

\section{Lifestyle of Year One Primary School Students with Moderate and Severe Astigmatism in Gua Musang: An Interpretative Phenomenological Analysis (IPA)}

\author{
Nurulain MZ1 , Syarmilla $C S^{1}$, Nur Liyana I', Aznor Azwan $A A^{1}$, Ahmad Ziad $S^{2}$, Suraida $A R^{1}$, Muhamad Afzam \\ Shah $A R^{3}$, Abdul Mutalib $O^{4}$ \\ 1Ophthalmology Department Hospital Sultan Ismail Petra, \\ ${ }^{2}$ Optometry Unit Hospital Gua Musang ${ }^{2}$ \\ ${ }^{3}$ Department of Optometry and Visual Science, Kulliyyah of Allied Health Sciences, International Islamic \\ University Malaysia \\ ${ }^{4}$ Department of Ophthalmology, University Sultan Zainal Abidin
}

Astigmatisme accounts approximately $13 \%$ of all refractive errors of the human eye. High degrees of astigmatism are associated with the development of amblyopia and myopia. This study explored the lifestyle among year one primary school students with moderate and high astigmatism in Gua Musang, Kelantan. Vision screening was conducted at 13 schools in Gua Musang District, Kelantan in 2019 and 2020. This qualitative study focused on lifestyle among year one primary school students. An in depth semi structured interview was conducted via telephone on nine parents. Students with moderate and severe astigmatism, lived in Gua Musang, referred from the school health team were purposely selected. The voice recordings were transcribed verbatim, coded and qualitatively analysed to generate relevant themes. Majority of parents raised issues regarding gadget usage during travelling, an avid reader, low lighting control while doing work, lack of outdoor activities and multiple usage of gadgets in daily living. Parents were aware of the risk of unhealthy lifestyle on their children's sight, particularly astigmatism. Programmes to increase public awareness of lifestyle modification and the harmful effects need to be highlighted. 


\title{
ORAL PRESENTATION
}

\section{ID89 OP20}

\section{Implementation of the Teleaudiology Approach in Special Education Service Centre: A Preliminary Study}

\author{
Rashid, Mohd Fadzil Nor $;$; Quar, Tian Kar²; Maamor, Nashrah²; Chong, Foong Yen²
}

\author{
${ }^{1}$ Audiology Program, School of Health Sciences, Health Campus, Universiti Sains Malaysia, Kubang Kerian, \\ Kelantan, Malaysia \\ ${ }^{2}$ Audiology Program, Center for Rehabilitation \& Special Needs, Faculty of Health Sciences, Universiti \\ Kebangsaan Malaysia, 50300 Kuala Lumpur, Malaysia
}

Teleaudiology is a branch of telehealth that provides audiological services through communication technologies simultaneously improving audiological efficiency. There are five educational audiologists in the Special Education Service Center (3PK) across Malaysia resulting in an increasing workload among them. Adopting teleaudiology may serve as an alternative to maximize audiology services in 3PK.

The study aimed to evaluate the satisfaction of educational audiologists and facilitators with teleaudiology services and to measure the internet connection speed in 3PKs. All education audiologists in Malaysia and five facilitators participated in this study. The facilitators consist of a speech-language therapist, a technician and three student management assistants. Essential audiological examinations were carried out on deaf and hard of hearing students using the teleaudiology approach. Upon completion of the test, participants were asked to fill out a seven Likert's scale questionnaire. Meanwhile, the internet connection speed for the Skype application was measured using the Ookla website. Ten participants were involved in this study, whereby $30 \%(n=3)$ men and $70 \%(n=7)$ women. The mean age for each subject group was $41.8 \pm 7.33$ (educational audiologists) and 34.8 \pm 4.38 (facilitators). The educational audiologist showed a high mean score value for the video otoscopy examination $(5.2 \pm 1.3)$, acoustic immittance $(5.6 \pm 1.5)$ and pure tone audiometry $(6.2 \pm 0.45)$. The facilitators also showed a high score with a similar score for each task $(6.2 \pm 0.84)$. Overall, the mean internet connection speeds for ping, download and upload values were $38.4 \pm 28.21 \mathrm{~ms}, 18.80 \pm 26.99 \mathrm{Mbps}$ and $9.81 \pm 26.99 \mathrm{Mbps}$, respectively. Teleaudiology services have been successfully implemented in 3PKs on hearing problems and have established professional collaboration. However, continued support and collaboration were required to ensure the sustainability of these services in 3PKs. 


\title{
ORAL PRESENTATION
}

\section{ID90 OP21}

\section{Scoping Review of Roles and Impacts of Online Fitness Communities on Adults during COVID-19 Pandemic}

\author{
Hasbullah, Husna ${ }^{1}$; Mohamad Fauzi, Fatin ${ }^{1}$; Shuhaimi, Puteri Alifah ${ }^{1}$; Glam Hadzir, Hafiz ${ }^{1}$; Tengku Mohd, \\ Tengku Amatullah Madeehah ${ }^{1}$; Norazmi, Abu Ubaidah Amir ${ }^{1}$.
}

${ }^{1}$ Faculty of Medicine and Health Sciences, Universiti Sains Islam Malaysia, Negeri Sembilan, Malaysia

Movement restriction due to COVID-19 pandemic has led to immense interest in physical activities in achieving a healthier lifestyle. Online fitness community (OFC) is defined as anything on the internet either apps or websites that have discussions, forums or sharing related to physical activities among users. As more people share the same fitness aims, more online fitness communities were created as a platform to achieve them. This scoping review aims to examine the roles and impacts of OFC during COVID-19 pandemic. The relevant articles were searched through PubMed. The following keywords and search terms were used: "online fitness communities", "SNS", "physical activities", "communities" and "COVID-19". Journal articles were included if they were primary studies, published in the English language, population were adult population and conducted during the COVID-19 pandemic. Articles were excluded if the population was disease specific. A total of 3703 articles were screened from the title and abstract level, of which 10 articles ( 2 quantitative, 5 qualitative and 3 mixed) from 11 countries mainly in Europe were assessed at full text. The mediums of delivery were websites, fitness devices, mobile applications and social media platforms. Majority of the articles highlighted OFC as a tool of internal or external motivation, networking and social support in influencing people to increase physical activity during COVID-19 pandemic. The studies showed that OFC resulted in positive social image and feedback, enhanced social connectedness between individuals, as well as increased wellbeing. Evidence also shows that OFC acts as a tool of fitness tracking and health information sharing leading to increase in awareness. This scoping review showed that OFC plays a significant role in providing motivational support, social support and health information for people to improve their overall wellbeing during COVID-19 pandemic. 


\section{ORAL PRESENTATION}

\section{ID96 OP22}

\section{Hypertension Self-care Activities Among Patients in Rural Pahang: A Preliminary Study}

Zakaria, Azizul Farid ${ }^{1}$; Ahmad Sharoni, Siti Khuzaimah'; Fauzi, Rosuzeita ${ }^{1}$.

${ }^{1}$ Centre for Nursing Studies, Faculty of Health Sciences, Universiti Teknologi MARA (UiTM) Selangor, Puncak Alam Campus, 42300 Selangor, Malaysia

Hypertension self-care activities have been considered a critical component in hypertension management, apart from allopathic medication adherence that contributes to blood pressure control. This study determined the level of self-care activities among patients with high blood pressure and examined demographic and health related characteristics differences between adherers and non-adherers to self-care activities. This cross-sectional study was conducted between January to March 2021 in four health clinics in Jerantut, specifically located in Pahang National Park and nearby village areas. Self-care activities were measured using the H-SCALE (Hypertension Self-Care Activity Level Effects) questionnaire, which was adapted to assess the behavioral activities recommended for optimal management of high blood pressure. The analysis involved a total of 181 hypertensive patients. More than half of participants reported adhering to medication recommendations and a nonsmoking lifestyle $(77.9 \%$ and $62.4 \%$, respectively). Following practices related to the dietary approach to stop hypertension (DASH) was less frequent, $(5.0 \%)$ and adherence to physical activity and weight management was also low $(28.7 \%$ and $31.5 \%$, respectively). Across the self-care activities, adherers were more likely to be female $(p<0.01)$ and overweight $(p<0.05)$. Non-adherers were more likely to be male $(p<0.01)$ and unemployed $(p<0.05)$. Many hypertension patients in Pahang rural areas especially male and unemployed groups still face challenges related to hypertension self-care, particularly with recommended dietary for hypertension, weight management, and physical activity. Health care providers should assess patients' hypertension self-care activities for more comprehensive treatment and management of the disease. 


\title{
ORAL PRESENTATION
}

\section{ID100 OP23}

\section{Propolis Mouthwash in Preventing Radiotherapy Induced Mucositis In Nasopharyngeal Carcinoma (NPC) Patients}

\author{
Hamzah, Mohd Hafiz ${ }^{1}$; Musa, Muhamad Yusri²; Abd Mutalib, Nor Shahida ${ }^{3}$; Ab Hamid, Siti Azrin ${ }^{4}$; Mohamad, \\ Irfan'. \\ ${ }^{1}$ Department of Otorhinolaryngology-Head \& Neck Surgery, School of Medical Sciences, Universiti Sains \\ Malaysia Health Campus, Kota Bharu, Kelantan, Malaysia \\ ${ }^{2}$ Department of Otorhinolaryngology-Head \& Neck Surgery, Advanced Medical and Dental Institute, Universiti \\ Sains Malaysia, Kepala Batas, Pulau Pinang, Malaysia \\ ${ }^{3}$ Department of Otorhinolaryngology-Head \& Neck Surgery, Hospital Sultan Abdul Halim, Sungai Petani, \\ Kedah, Malaysia \\ ${ }^{4}$ Department of Biostatistics and Research Methodology, Universiti Sains Malaysia Health Campus, Kota \\ Bharu, Kelantan, Malaysia
}

Mucositis is one of the debilitating complications of treatment radiotherapy in Nasopharyngeal Carcinoma (NPC). Mucositis can lead to a patient's malnutrition due to poor oral intake. The cancer treatment protocol might be postponed due to the patients' malnutrition. Until now no specific treatment to prevent radiotherapy induced mucositis in NPC patients. To evaluate effectiveness of propolis mouthwash in preventing radiotherapy induced mucositis in NPC patients. This is a double arm, prospective, randomized control trial (RCT) with intervention involving 17 patients diagnosed with NPC, attending ORL-HNS clinic in Advanced Medical \& Dental Institute (AMDI). The effectiveness of propolis mouthwash was assessed by Repeated Measure ANOVA. All 17 NPC patients were randomly divided into propolis group (intervention) and normal saline group (placebo). 10 patients were given propolis mouthwash and another 7 patients were given normal saline mouthwash. Comparison of adjusted mean and 95\% confidence interval of mucositis grading score between propolis mouthwash and normal saline show at week two, $0.10(-0.32,0.52)$ vs $1.14(0.64,1.64)$, week four, $0.50(0.13,0.87)$ vs $2.00(1.56,2.44)$, and week six, $1.20(0.93,1.47)$ vs $2.86(2.53,3.18)$ respectively. Repeated Measure ANOVA showed significant increasing improvement of mucositis grading between propolis and normal saline group $(p<0.001)$. Propolis mouthwash is proven to be effective in reducing the severity of radiotherapy induced mucositis in NPC patients and it is safe to be used. Therefore, its use is recommended as an adjunct treatment during the treatment protocol of NPC. 


\title{
ORAL PRESENTATION
}

\section{ID112 OP24}

\section{Refractive Error Among Learning Age Group Prior to, and During COVID-19 Pandemic}

\author{
Zainol, Norlelawati ${ }^{1}$; Mokhtar, Azlina ${ }^{1}$; Muna'aim, Maimunah ${ }^{1}$; Khairudin, Muhammad Najmi ${ }^{1}$; Ghani, Nor \\ Diyana²; Awaluddin, S Maria ${ }^{3}$. \\ ${ }^{1}$ Department of Surgery (Ophthalmology), Faculty of Medicine and Health Sciences, Universiti Sains Islam \\ Malaysia, Negeri Sembilan, Malaysia \\ ${ }^{2}$ Our Optometrist, Klinik Pakar Kesihatan USIM, Negeri Sembilan, Malaysia \\ ${ }^{3}$ Institute for Public Health, National Institutes of Health, Ministry of Health, Selangor, Malaysia
}

Refractive error is the most common cause of visual impairment in the world. Uncorrected refractive error will have a significant effect on education and quality of life among our young age group. COVID-19 pandemic leads to more online learning and reduced outdoor time which expose the young population to the risk of refractive error progression. To evaluate the refractive error among the learning age group prior to, and during COVID-19 pandemic era. This is a descriptive and bivariate retrospective data analysis of refraction among learning age groups (3-year-old to 22-year-old) from January 2019 till June 2021 at Klinik Pakar Kesihatan USIM. The incidence of refractive errors was compared from pre COVID-19 pandemic and during COVID-19 pandemic. The eye with the worst spherical equivalent was taken for analysis. A total of 269 patients (Pre COVID, $n=106$ vs During COVID, $n=163$ ) were included in this study which comprises 135 males and 134 females. Most of the patients were from 7-year-old to 12-year-old age group (62\%). Myopia was found in $78 \%$ while only $22 \%$ were hyperopic. The highest spherical equivalent was -15 Diopter for myopia and +3Diopter for hyperopia. The proportion of myopia increased from $73 \%(n=77)$ to $82 \%(n=133)$ during COVID-19 pandemic. There is a positive low correlation between myopia level and age with spearman's rho of 0.35 . The COVID19 pandemic brings about a rising trend of refractive error among our young learning age population. The movement control order reduces the outdoor activity and concurrently increases the screen exposure time. Awareness of healthy eye habits would reduce progression of refractive error. A prospective study would prove if there is an actual worsening of myopia in children due to the above factors. 


\title{
ORAL PRESENTATION
}

\section{ID125 OP26}

\section{Semi-automating a Mandarin Paediatric Speech Perception Test for Use by Non-Mandarin Speaking Audiologists: A Preliminary Study}

\author{
Foong Yen Chong ${ }^{1}$; Cila Umat ${ }^{1}$; Kah Kei Mak ; Yan Jin Goh'; Badrulzaman Abdul Hamid²; and Bee See Goh ${ }^{3}$. \\ ${ }^{1}$ Audiology Programme, Centre for Rehabilitation and Special Needs Studies, Faculty of Health Sciences, \\ Universiti Kebangsaan Malaysia. \\ ${ }^{2}$ Speech Science Programme, Centre for Rehabilitation and Special Needs Studies, Faculty of Health Sciences, \\ Universiti Kebangsaan Malaysia \\ ${ }^{3}$ Department of Otorhinolaryngology-Head and Neck Surgery (ORL-HNS), Faculty of Medicine, Universiti \\ Kebangsaan Malaysia.
}

Speech perception testing is an important assessment tool for audiological diagnostic and intervention programs among the pediatric population. Speech perception tests are language specific and usually require audiologists to have a good command of a particular language for the test stimuli presentation and scoring. These limit the clinical application of Mandarin speech perception tests on Mandarin-speaking children as the majority of the audiologists in Malaysia are non-Mandarin speakers. This study aimed to develop a preliminary semi-automated digitized speech perception test application and manual, used by the non-Mandarin speaking audiologists to test Mandarin-speaking children via an online mode. This crosssectional study had three phases. In Phase I, needs assessment via focus group discussions (FGDs) was conducted among 20 participants (audiologists and Audiology students). In Phase 2, an online, semi-automated test application and manual were developed and reviewed. In Phase 3, content validity evaluation of the application and manual was piloted among six audiology students by using an adapted Suitability of Assessment Material form with confidence and perception ratings.14 main themes emerged from the needs assessment. Four themes with the highest number of occurrences include 'test procedure' (25.7\%), 'tester' (20.7\%), 'demo/training' (9.1\%), and 'test items' $(8.7 \%)$. The digitized semi-automated test application was developed by using PowerPoint with hyperlinks to present stimuli and capture picture-pointing responses. Preliminary results of the item-level content-validity index scores were $\geq 0.83$ for the semi-automated test application, and most items for the test manual. The confidence and perception ratings were 3.96/4.00 for the test application and 3.50/4.00 for the test manual. Results of FGDs supported the needs for developing a semi-automated speech perception test application and manual. The preliminary test application and manual have good content validity and can potentially be an online assessment tool to be used by non-Mandarin speaking audiologists. 


\title{
ORAL PRESENTATION
}

\section{ID135 OP27}

\section{The Prevalence of Computer Vision Syndrome, Its Associated Risk Factors and Awareness Among Undergraduate University Students: A Systematic Review}

\author{
Mohd Azam, Ummi Amani'; Mohd Farezal, Nurul Amarina'; Pazudin, 'Imran; Zulkefli, Afiq Zakwan'; Che Abd \\ Aziz, Uthman-1; Mohmad Sanip, Aina Nabilah-1; Abdul Aziz, Anis-1; Ahmad Hamizi, Mohamed Aiman-1; Khair, \\ Muhammad Basyir-1; Jasny, Faiq Fauzani-1; Razali, Khairul Redwan-1; Mohd Salihuddin, Aina Najeehah 1 ; Yazid, \\ Anisah ${ }^{1}$; Baniamin, Nur Shafini ${ }^{1}$; Mohd Shaharudin, Shahidah ${ }^{-} ;$Mukhadzir, Afiqah $\stackrel{1}{-}$; Tajulazhar, Salma ${ }^{1}$; Wan

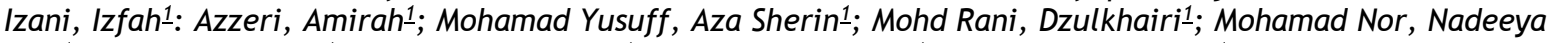

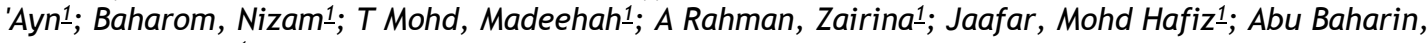 \\ Mohamed Fakhri 1
}

1 Public Health Unit, Department of Primary Health Care, Faculty of Medicine and Health Sciences, Universiti Sains Islam Malaysia

Digital device usage has increased substantially in recent years and has been used extensively as an educational tool. However, prolonged used of these devices pose a higher risk of acquiring visual-related symptoms known as Computer Vision Syndrome (CVS), which can lead to reduced work productivity. We aimed to determine the prevalence of CVS, its associated risk factors and awareness among undergraduate university students. A systematic search was performed using three electronic databases PubMed, SCOPUS and EBSCOhost. The included terms in the search strategy were 'undergraduate student', 'computer exposure', 'risk factor', 'sociodemographic', 'prevalence', 'awareness' and 'computer vision syndrome'. The selection and identification of relevant studies were screened according to PRISMA guidelines. The Newcastle-Ottawa Scale was used to evaluate the quality of these studies. Initial search identified 34,300 studies and 36 studies (35 crosssectional and 1 cohort) were included after applying the inclusion and exclusion criteria. The prevalence of CVS among undergraduate university students ranges from $18.7 \%$ to $98.7 \%$. Eye strain was reported as the most common ocular symptom, while headache was the commonest for extraocular symptoms. Factors that were associated with CVS were age, gender, refractive errors, duration of computer usage, distance from screen, use of anti-glare, level of computer screen from eyes, frequency of breaks, posture, type of screen and room illumination. The awareness of computer vision syndrome from the studies varied from $8.6 \%$ to $63 \%$. Our result acknowledges that the prevalence of CVS, its associated factors and awareness are varied between studies. Further studies are needed to ascertain the causality between CVS and its associating factors, as well as efforts to increase CVS awareness amongst university students. 


\section{ORAL PRESENTATION}

\section{ID139 OP28}

\section{Community-Developed Video Clips as An Effective Risk Communication Tool on COVID-19 Preventive Measures by The Indigenous Orang Asli Communities}

Mohd Dzulkhairi Mohd Rani ${ }^{1}$, Hana Maizuliana Solehan ${ }^{1}$, Nurul Azmawati Mohamed ${ }^{1}$, Nadeeya'Ayn Umaisara Mohamad Nor', Zatul-'Iffah Abu Hasan'1, Suhaila Sanip'

${ }^{1}$ Faculty of Medicine and Health Sciences, Universiti Sains Islam Malaysia, Nilai, Negeri Sembilan

The best way to prevent and slow down the transmission of COVID-19 is to be well informed about the mechanism of infection and its preventive measures. Risk communication is a key component of public health interventions during an outbreak that enable effective communication with the public, engaging with communities, local partners, and other stakeholders. This study aims to develop an effective risk communication tool in indigenous language to promote COVID-19 preventive measures among the Orang Asli community in Malaysia. The Community-Based Participatory Research (CBPR) approach was used in this study. Our collaborators for this study are Tengku Ampuan Afzan Teacher Training Institute and the Department of Indigenous Affairs Malaysia. Three Orang Asli Communities in several locations were chosen to participate in this study. In-depth interviews and focus group discussions were used as a baseline for the study. Health promotional video clips were developed by the community themselves in their own indigenous languages and local settings as part of the CBPR approach. The video clips were sent to expert reviewers to validate their contents. The themes of the clips include the importance of hand hygiene, the use of face masks and the COVID-19 vaccine. The use of everyday terms and in their own settings allow the community to easily understand and digest the contents. Dissemination of the video clips among the community members was done through social media and messaging platforms. The reflections from the developers of the clips showed that in the process of developing the clips, they learn new information on COVID-19. The development and use of health promotion video clips as risk communication tools among indigenous Orang Asli are most effective if developed in their own language, settings, and community members. 


\title{
ORAL PRESENTATION
}

\section{ID144 OP29}

\section{Association Between Blastocystis sp. Infection and Type 2 Diabetes Mellitus in Adults in Malaysia: A Pilot Case Control Study}

\author{
Mohd Shaari, Nurul Saadah ${ }^{1}$; Wan Shahida, Wan Sulaiman ${ }^{1}$; Mohd Rahman, Omar ${ }^{1}$; Nadeeya'Ayn Umaisara \\ Samseh Abdullah, Noradilah ${ }^{1}$ \\ ${ }^{1}$ Faculty of Medicine and Health Sciences, Universiti Sains Islam Malaysia, 71800 Nilai, Negeri Sembilan, \\ Malaysia
}

An emerging of tropical disease caused by opportunistic protist, Blastocystis sp. had been a medically important among immunocompromised patients with potentially lead to irreversible complication. However, little is explored about the prevalence of Blastocystis sp. infection in patients with type 2 diabetes mellitus (T2DM) specifically in Malaysia. The purpose of this pilot study was to generate the preliminary proportion and the association of Blastocystis sp. infection in type 2 diabetic patients compared to healthy individuals. This case control study was conducted at Universiti Kebangsaan Malaysia Medical Centre, involving 21 patients and 20 healthy controls. Stool samples were collected from the patients after the consent had been obtained. The samples were examined microscopically by using in vitro culture (IVC) technique by Jones medium. While, the data were analyzed using descriptive and chi-square tests, with a $\mathrm{p}$-value $\leq 0.05$ considered statistically significant. Out of 41 samples, 26 (63.4\%) were detected with Blastocystis sp. infection among diabetic patients and healthy individuals. Infection with Blastocystis sp. was more prevalent in diabetic patients $(81 \%)$ than in controls $(35 \%)$. It was found that there was a significant association between in Blastocystis sp. infection and groups of participants with higher prevalence among diabetic patients and control as compared to control group, $\mathrm{p} \leq 0.05$. In addition, the infection of Blastocystis sp. has been significantly associated with abdominal pain symptom with $p \leq 0.05$. The pilot study results demonstrated that diabetic patients are at greater risk of contracting Blastocystis sp. infection than the healthy population. In conclusion, a large case control study with enrolment of diabetic patients and controls at health facilities is needed to reinforce the relationship between Blastocystis sp. and diabetic patients. The possible risk factors should be interpreted rigorously to minimize the infection mainly in immunocompromised patients. 


\section{ORAL PRESENTATION}

\section{ID145 OP30}

\section{The use of Virtual OSCE (vOSCEs) in Medical Education: A scoping review}

Mohd Bakhit, Nour Hanan Daniah ${ }^{1}$; Muhammad Saifullah, Ahmad Anwaar ${ }^{1}$; Jailani, Ruhi Fazlyana

${ }^{1}$ Faculty of Medicine and Health Sciences, Universiti Sains Islam Malaysia, Nilai, Negeri Sembilan, Malaysia

COVID-19 has globally changed medical education delivery. Virtual Objective Structured Clinical Examination (vOSCEs) is increasingly being used by various institutions worldwide to ensure the continuity of medical education and assessments. The purpose of this scoping review is to provide a comprehensive overview of VOSCEs barriers and solution; sequentially identifying the gaps which could suggest additional potential study and appraisal. This review adopted the fivestage framework outlined by Arksey and O'Malley. 669 articles were identified by using the key descriptors via Scopus, PubMed, Science Direct and ResearchGate. The process of article selection followed the Preferred Reporting of Items for Systematic Reviews and Meta-Analyses (PRISMA) Statement. Articles were screened and assessed for eligibility. Duplicates and irrelevant articles were removed. A final yield of 43 articles were included in this review. Data were extracted to summarize, collate, appraise the quality and a narrative account is constructed based on the findings. Common themes that emerged across the study designs with respect to challenges of VOSCE were technical problems, psychological stress, inability to access physical examination and integrity of the examination. Solutions included multi-expertise collaboration, communication, guiding the patient to do physical examination (PE) maneuver virtually and PE narration, training of students and staffs, mock OSCE, and debriefing session. However, certain areas such as digital professionalism, telemedicine skills, validity of vOSCE and PE narration require further research to scrutinize the feasibility and provide guidelines for VOSCE. In conclusion, the COVID19 pandemic is not the first and will not be the last pandemic. Medical education structure must be versatile and well equipped in facing such challenges. With advancement of technology and skills, vOSCE may prove to be a robust and reliable tool in medical education. 


\section{ORAL PRESENTATION}

\section{ID147 OP31}

\section{Prevalence of Computer Vision Syndrome and the Associated Factors Among Undergraduate University Students in Malaysia}

Zainol, Mohamad Akmal Arif ${ }^{1}$; Salahuddin, Nurul Izzati풀 Md Mokhsen, Siti Nuraisyah ${ }^{1}$; Mohd Sabri, Muhammad

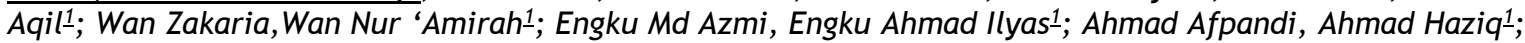
Zulkifli, Muhammad Irfan ${ }^{1}$; Normaly Kamal, Siti Fatimah-1; Anuar, Aida Syafiqah ${ }^{\prime}$; Noor Zari, Muhammad Aliff Haziq ${ }^{-}$; Md Haris, Amirul Ameer $\stackrel{1}{-}$; Zakaria, Muhammad Zaim ${ }^{1}$; Nor Alkose, Muhammad Naqib Afifuddin-1; Aman

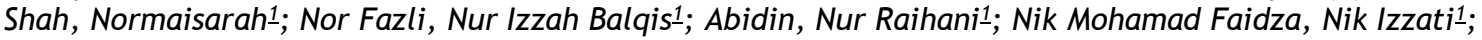
Elias,Noor Eza Shazwana ${ }^{1}$; Wan Azemi, Wan Nur Aima Amirah; Azzeri, Amirah; Mohamad Yusuff, Aza Sherin; Mohd Rani, Dzulkhairi1; Abu Baharin, Fakhri풀 Mohamad Nor, Nadeeya 'Ayn¹; Baharom, Nizam¹; T Mohd, Madeehah풀 A Rahman, Zairina풀 Jaafar, Mohd Hafiz ${ }^{1}$

1Faculty of Medicine and Health Sciences, Universiti Sains Islam Malaysia, Bandar Baru Nilai , 71800, Nilai, Negeri Sembilan, Malaysia

Computer vision syndrome (CVS) is common health problem that associated with computer/gadget usage. Prevalence among undergraduate students globally ranging from $18-97 \%$ and associated with multiple risk factors. This study aims to determine the prevalence and associated factors (sociodemographic, computer-related factors and knowledge) of CVS among undergraduate university students. An online survey using google form was conducted involving undergraduate university students (Malaysian and international students) of any courses from all universities in Malaysia. CVS in this study was defined as respondents reported three or more symptoms (dry eyes, blurring of vision, eye pain, headache, neck and/or shoulder pain) resulted from using computer/tablet/ipad/handphone. Level of knowledge for CVS was categorised as high (marks above median, 76 marks) or low (marks below median). A total of 463 students responded in this study. The prevalence of CVS in this study was $43.6 \%$. The highest reported symptom was neck and/or shoulder pain $(86.0 \%)$ followed by headache $(62.0 \%)$, burning eye sensation $(52.3 \%)$, dry eyes (46.4\%) and blurring of vision (45.8\%). The lowest reported symptom was redness of eyes (29.2\%). Majority of the students (54.0\%) had low level of knowledge about CVS. Factors significantly associated with CVS were female $(\mathrm{OR}=1.833,95 \% \mathrm{Cl}=1.229$ 2.734, $\mathrm{p}=0.003)$, wearing eyeglasses $(\mathrm{OR}=2.150,95 \% \mathrm{Cl}=1.479-3.126, \mathrm{p}<0.001)$ and more than 6 hours duration of devices usage per day $(O R=2.425,95 \% \mathrm{Cl}=1.442-4.076$, $\mathrm{p}=0.001)$. In conclusion, almost half of the undergraduate students in this study had experienced computer vision syndrome. The significant contributing factors were being female gender, wearing eyeglasses and spent more than 6 hours per day on computer/gadget. Majority had low knowledge on CVS, thus relevant authorities were recommended to educate students on CVS and incorporate this topic in their curriculum. 


\title{
ORAL PRESENTATION
}

\section{ID157 OP32}

\section{Knowledge, Attitude and Practice of Health Risks of Video Gaming Towards Eyes, Hearing and Body Posture Among Malaysian Parents with Children Under 18 Years Old}

\author{
Hassan, Nor Eyzawiah'; Muhammad Saifullah, Ahmad Anwaar'; Mohd Bakhit, Nour Hanan Daniah'; Abu Hasan, \\ Zatul 'Iffah'; Abdul Muna'aim, Maimunah'; Mohamad Nor, Nadeeya'Ayn Umaisara'
}

Faculty of Medicine and Health Sciences, Universiti Sains Islam Malaysia (USIM), Persiaran Ilmu, Putra Nilai, 71800. Nilai, Negeri Sembilan, Malaysia

The popularity of video gaming and esports is growing at a rapid pace worldwide and associated with various health hazards. The implementation of movement control order (MCO) which restricted people from moving during the COVID19 pandemic may exacerbate this problem. A descriptive cross-sectional study was designed to assess the knowledge, attitude, and practices (KAP) of Malaysian parents with children under 18 years old in relation to the health risks of video gaming towards eyes, hearing and body posture and role of sociodemographic factors. 355 respondents participated in this study. Self-administered questionnaire was used to assess the knowledge, attitude, and practice of health risks of esports and shared via social media applications to reach the target population. Data analysis was carried out using SPSS version 28. Descriptive analysis was used to analyse the qualitative variables while quantitative variables were summarised using mean \pm Standard Deviation $( \pm S D)$, and multiple logistic regression analysis was also done. On average, respondents scored higher in attitude score followed by knowledge and practice scores. The average attitude score was 73.65\% (95\% Cl: [72.75, 74.55\%]) compared to $61.13 \%$ for knowledge $(95 \% \mathrm{Cl}$ : [59.20, 63.13\%]) and $60.64 \%$ for practice $(95 \% \mathrm{Cl}$ : [59.05, 62.22\%]). The results suggest that overall, Malaysian parents have poor knowledge, attitude, and practice regarding the health risks of video gaming towards eyes, hearing and body posture. Parents with higher education level are more likely to have good knowledge, and those with no history of children entering esports tournaments are more likely to have positive attitude. The positive attitude predicts good practice among the parents. Parents play a crucial role in mediating video gaming among children. Public campaigns should be organized to improve the knowledge, attitude and practice of parents and other stakeholders on the health implications of video games. 


\section{ORAL PRESENTATION}

\section{ID168 OP33}

\section{Ethical \& Legal Issues in Remote Medical Consultation - Advocating Regulatory Oversight on Telemedicine in Malaysia}

Yunus, Aimi Mohd' ${ }^{1}$; Katiman, Helwa Husna ${ }^{1}$; Muuti, Muhamad Zaid ${ }^{1}$

${ }^{1}$ Department of Medical Ethics and Law, Faculty of Medicine, Universiti Teknologi MARA, Sungai Buloh, Malaysia

A 1997 Malaysia's Telemedicine Blueprint envisioned transforming the country's healthcare system into digital medicine by 2020. There is little use of the unenforceable Telemedicine Act 1997 for remote medical consultation between patients and doctors, albeit through continuous efforts. Prior to the COVID-19 pandemic, the Malaysian Medical Council prohibits doctors from prescribing medications to callers over the phone or any other electronic device. However, the pandemic has forced Telemedicine to be adopted to deliver continuous medical care in a timely and safe manner that conventional access could not provide. Text-based, voice-based, and video-based communication is utilized to perform remote medical consultations. It does, however, raise ethical and legal concerns. This study aims to identify and analyze the ethical and legal issues surrounding remote medical consultation in Malaysia. This study adopts a doctrinal methodology. It was found that the unenforced act has resulted in unregulated Telemedicine Platforms in Malaysia. The lack of Telemedicine practice guidelines for medical practitioners poses numerous legal concerns, such as the inability to perform physical examinations, exacerbated by a lack of training and competency to treat patients via Telemedicine. This circumstance further raises issues about consent, caller identification, breaching of privacy and confidentiality, and preservation of medical records. While there are numerous issues in Telemedicine, this practice might be deemed ethical. It provides access to medical care when the conventional clinic visit is impossible, as some level of care is better than no care at all. Teleconsultation will not replace face-to-face consultations, but rather, it is an additional service for the patients. A practice guideline for the medical practitioner is necessary to encompass the holistic approach in teleconsultation, where appropriate training is not acquired. Telemedicine Platforms must be held accountable and should not serve as a mere "communication medium" while passing all the responsibility to a medical practitioner. 


\title{
ORAL PRESENTATION
}

\section{ID187 OP34}

\section{Use of 3D based Virtual Learning Resources in Teaching Anatomy: A Scoping Review}

\author{
$\underline{\text { Ab Rahman, Siti Soraya }}$; Rashid, Zamir ${ }^{2}$
} ${ }^{1}$ Faculty of Medicine and Health Sciences, Universiti Sains Islam Malaysia, Negeri Sembilan, Malaysia
${ }^{2}$ Innoveam Sdn. Bhd., Kuala Lumpur, Malaysia

3D based Virtual learning resources (3DVLRs), experienced through virtual reality and augmented reality technologies, are becoming more widely used in medical education, including in anatomy. This review aims to identify the types of 3DVLRs that are used in anatomy education and to determine their effectiveness. Using the Joanna Briggs Institute manual for scoping reviews as guidance, six databases and reference lists of relevant systematic reviews were searched from 2000 to August 2021. Any type of study involving the use of 3DVLRs in anatomy education were included. Screening and data extraction were conducted by two reviewers independently. Frequency and thematic analyses were conducted. A total of 253 abstracts were screened, with 70 full papers meeting the eligibility criteria. The interest in implementation of such technologies in anatomy education has increased significantly since 2016. Various types of 3DVLR technologies were used, from simple $3 \mathrm{D}$ visualization to highly interactive immersive Virtual Reality with haptic feedback. Most of the findings point to positive learning outcomes when compared to traditional learning approaches, despite some significant drawbacks identified, particularly high cognitive loading and the importance of stereopsis and realism. However, the implementations were mostly done in an experimental setting, where students were only able to use the 3DVLR for a given period. None of the papers mentioned about the quality of the 3D content visualized and how this can be quantified. The benefits of 3DVLR may become more significant if they are more accessible to the students and can be used for the duration of the anatomy course, and if the quality of the instructional design and 3D visualizations of the 3DVLRs can be further enhanced. 3DVLRs experienced through virtual reality and augmented reality technologies have become more common and are shown to be effective in anatomy education. 


\section{ORAL PRESENTATION}

\section{ID193 OP35}

\section{Distribution and Progressivity of Out-of-Pocket Payments for Health Care: Evidence from the Malaysian Household Expenditure Survey}

Abu Baharin, Mohamed Fakhri' ${ }^{1}$; Juni, Muhamad Hanafiah²; Abdul Manaf, Rosliza²

${ }^{1}$ Public Health Unit, Department of Primary Health Care, Faculty of Medicine and Health Sciences, Universiti Sains Islam Malaysia

${ }^{2}$ Department of Community Health, Faculty of Medicine and Health Sciences, Universiti Putra Malaysia

Equity or fairness in the distribution of health resources is essential to a country's journey towards universal health coverage. Out-of-pocket (OOP) payments are an inequitable mechanism for health financing as its high share of total health expenditures poses a risk of catastrophic healthcare expenditures. This study was conducted to assess the distribution and progressivity of OOP payments for health care in Malaysian households. Data from the Malaysian Household Expenditure Survey (HES) for the year 2014/2015 were used for this retrospective cross-sectional study, which included 14,473 households. The distribution and progressivity of OOP payments were measured using the proportionality approach and the Kakwani progressivity index $(\mathrm{KPI})$, respectively. Progressivity is measured at the national level, by region, by urban and rural strata, and by ethnic group. The average share of Malaysian' OOP payments for health care from total household expenditure was $1.65 \%$. The proportions increased across households' socioeconomic quintiles, from $1.03 \%$ made by the poorest $20 \%$ to $1.86 \%$ by the richest $20 \%$. The distribution of OOP payments in Malaysia was more concentrated among the richer households with a positive KPI of 0.0910 , indicating progressive OOP payments for health care. OOP payments were also progressive across all Malaysian regions, urban and rural strata, and ethnic groups. It was most progressive in Sarawak region, rural strata, and among ethnic Bumiputera with KPI of $0.0959,0.1464$ and 0.109 respectively. This study has shown that OOP payments for healthcare in Malaysia are progressive and more concentrated among the better-off, hence equitable. There is a need for further studies using data from subsequent years to analyze the progressivity trend of OOP payments, as well as other inequality analyses, for example, to assess the risk of catastrophic health expenditure due to OOP payments. 


\title{
ORAL PRESENTATION
}

\section{ID197 OP36}

\section{Evolution in Technology: Innovation of Tooth brushing Training among School Children}

\author{
Siti Zuriana $\mathrm{MZ}^{1}$ \\ ${ }^{1}$ Oral Health Division Federal Territory Kuala Lumpur \& Putrajaya, Malaysia
}

Tooth brushing is an effective way of removing plaque, preventing gingivitis, and managing dental caries. The effectiveness depends not only on the type of toothbrush but also the proper brushing technique. By advanced technology, the teaching of proper brushing technique could be created. This study was aimed to assess the effectiveness of tooth brushing training using "Robotic" among the primary school children in improving the knowledge of tooth brushing. This observational study involving primary schoolchildren aged 8 - 11 years old was conducted in the Federal Territory of Kuala Lumpur \& Putrajaya from June 2019 to February 2020. The effectiveness was defined as an improvement in the knowledge of tooth brushing technique before and after the training using "Robotic". Training using "Robotic" included demonstrating tooth brushing with programmed voice. Tooth brushing training was performed by teachers during the Health Education subject with the assistance of "Robotic". Knowledge and plaque score that was measured using O'Leary Index was assessed at the beginning and after 6 months later. Data were analyzed by chi squared and t-test. Four hundred and forty-four primary school children participated in this study consisting of $51 \%$ male and $49 \%$ female. Majority of them were Malay with the mean age of 9.54-year-old. There was significant improvement of knowledge $(p<0.05)$ especially observed in these two questions: 1. "How many times do you need to brush your teeth (61\%)" and 2. "How many times is the tooth brushing movement for each tooth surface (86.9\%)". The plaque score was noted to have improved significantly during the second examination (67.8\%). Tooth brushing training by "Robotic" was effective in improving knowledge. This finding is important to support the method of training in the new norm utilizing less human contact and to improve future oral health education programs. 


\title{
POSTER PRESENTATION
}

\section{ID6 PP001}

\section{Is the Drone Cost Effective? An Economic Evaluation of Drone versus Ambulance for Blood Products Transportation}

Afiq Hidayat M, Azma RZ, Aniza I, Ismail MS, Rahana AR, Mahdy ZA

\author{
${ }^{1}$ Department of Pathology, Faculty of Medicine, Universiti Kebangsaan Malaysia Medical Center (UKMMC), \\ Kuala Lumpur, Malaysia. \\ ${ }^{2}$ Department of Community Health, Faculty of Medicine, Universiti Kebangsaan Malaysia Medical Center \\ (UKMMC), Kuala Lumpur, Malaysia. \\ ${ }^{3}$ Department of Emergency Medicine, Faculty of Medicine, Universiti Kebangsaan Malaysia Medical Center \\ (UKMMC), Kuala Lumpur, Malaysia. \\ ${ }^{4}$ Department of Obstetrics \& Gynaecology, Faculty of Medicine, Universiti Kebangsaan Malaysia Medical \\ Center (UKMMC), Kuala Lumpur, Malaysia.
}

Drone or Unmanned Aerial Vehicle (UAV) is the latest technological innovation that may revolutionize medical transportation globally, including blood products transportation between blood bank services and health care facilities. However, its economic evaluations are scant and insufficient, whilst its cost effectiveness remain controversial. This study strived to answer the doubts of economic viability of the drone for blood products transportation by comparing the cost effectiveness between the drone and the ambulance. The setting was within a developing nation. A comparative economic analysis of emergency blood products transportation using drone versus ambulance between the Sabah Women and Children Hospital (SWACH) and the Queen Elizabeth II Hospital (QEH2) on Borneo Island was conducted using the Cost Effectiveness Analysis (CEA) technique. Subsequently, the Incremental Cost Effectiveness Ratio (ICER) was calculated using the travel time as a non-clinical denominator. A round trip of blood products transportation from SWACH to QEH2 in a clinical emergency cost RM1,266.02 (USD307.09) using an ambulance, compared to RM1,313.28 (USD 319.36) using a drone. The CER of ambulance transportation was RM37.23 (USD9.05) per minute whilst the CER of drone transportation was RM72.96 (USD17.74) per minute. The ICER of drone versus ambulance revealed a negative value of -2.23 . The current technological development showed that the drone cost more per minute compared to the ambulance in the transportation of blood products. Nonetheless, the significantly shorter transport time of the drone versus ambulance counterbalanced its cost. Thus, we believed that there is great economic potential for drone usage in the future for blood products transportation in developing nations as the drone price decreases, the operational lifespan increases, and its velocity improves. 


\section{POSTER PRESENTATION}

\section{ID7 PP002}

\section{COVID-19 Pandemic and Face Mask Usage: Prevalence and Factors Associated in Malaysian Medical School}

Nurul Hayati Mohamad Zainal ${ }^{1}$, Aishwarya Sivakumar ${ }^{2}$, Ahmad Muaz Ibrahim² ${ }^{2}$ Dayana Aida Azhar ${ }^{2}$, Nurul Huda Mohd Nor $^{1,3 *}$.

${ }^{1}$ Department of Human Anatomy, Faculty Medicine and Health Sciences, Universiti Putra Malaysia, 43400 Serdang, Selangor, Malaysia

${ }^{2}$ Third Year Medical Students, Faculty Medicine and Health Sciences, Universiti Putra Malaysia, 43400 Serdang, Selangor, Malaysia

3Institut Penyelidikan Penuaan Malaysia (MyAgeing), Universiti Putra Malaysia, Selangor, Malaysia

COVID-19 is a contagious disease caused by a newly discovered coronavirus strain. Anxiety, anticipation, and lack of knowledge in the previous experience on severe acute respiratory syndrome and $\mathrm{H} 1 \mathrm{~N} 1$ pandemic have a substantial impact on significant public health behaviors. This includes wearing a face mask in public spaces. We investigate the prevalence of face mask usage and its associated factors during Covid-19 amongst students and staff of Faculty Medicine and Health Sciences (FMHS), Universiti Putra Malaysia (UPM). Using a cross-sectional study, 220 respondents participated in an electronic version of a self-administered questionnaire. Analyses were performed using the Chi-square test, IBM SPSS Statistics 25 , with statistical significance at $p<0.05$. Out of the 220 respondents, 197 $(89.5 \%)$ reported that they wore face masks all the time. Most of the respondents, $90.3 \%$ had a high level of precautionary measures against Covid-19, $89.6 \%$ selected the internet as their source of information and $90.9 \%$ of the respondents had a good attitude level towards measures against COVID-19. There were significant associations between hygienic practices $(p=0.001)$, source of information (internet) $(p=0.025)$, and attitude towards Covid-19 $(p=0.001)$ with face mask usage. In conclusion, this study demonstrated a high prevalence $(89.5 \%$ ) of face mask usage during the Covid- 19 outbreak amongst students and staff of FMHS, UPM. 


\section{POSTER PRESENTATION}

\section{ID12 PP004}

\section{COVID-19 vaccine hesitancy among public in Malaysia: a cross- sectional survey}

Aws hashim Ali Al-Kadhim!', Faizah Abdul Fatah ${ }^{1}$, Normaliza Ab Malik ${ }^{1}$, Sarah Taha Yousif ${ }^{2}$, Azrul Hafiz Abdul Aziz $^{1}$, Syatirah-Najmi Abdullah ${ }^{1}$, Azlan Jaafar ${ }^{1}$

${ }^{1}$ Lecturer, Faculty of Dentistry, Universiti Sains Islam Malaysia

${ }^{2}$ WeCare Medical clinic, Kuala Lumpur, 55100, Malaysia

Vaccination is an effective strategy to reduce the burden of COVID-19, but its success is contingent on vaccine acceptance. To avoid the vaccination program failing, it is necessary to address vaccine-apprehensive persons' worries. The purpose of this study was to determine the level of acceptance rate towards COVID-19 vaccine among Malaysians. An online questionnaire was distributed to 599 respondents via a snowball sampling method among Malaysians aged 18 years and above. Out of 599, acceptance rate was $89.2 \%$ with majority of the respondents were female $(68.4 \%)$, aged between 20 to29 and 30 to 39 years (30.2\%), married (57.4\%), Muslim (61.1\%), Selangor residence (34.3\%), bachelor's holder $(63.3 \%)$, non-health worker $(53.1 \%)$, and healthy individuals (59.9\%). The most likely reason for refusal of taking COVID19 vaccine was due to the long-term effect of vaccine $(49.6 \%)$, gender $(p=0.017)$ and occupation $(p=0.038)$ of the respondents were found to be significantly associated with the likelihood of taking the COVID-19 vaccine. The findings showed that Malaysians have a high level of acceptance for the COVID-19 vaccine. Despite the high acceptance rate, it is still vital to address concerns among the vaccine's sceptics by establishing faith in the vaccine's safety and effectiveness through proper vaccine information. 


\title{
POSTER PRESENTATION
}

\section{ID15 PP005}

\section{Child Abuse During the COVID-19 Pandemic}

\author{
Ghani, Syamsul'; Yahaya, Yafizah²; Ganason, Anu Suria²; Syed Mohideen, Fathima Begum² \\ ${ }^{1}$ Faculty of Medicine and Health Science, Universiti Sains Islam Malaysia, Nilai, Malaysia \\ ${ }^{2}$ Family Medicine Unit, Faculty of Medicine and Health Science, Universiti Sains Islam Malaysia, Nilai, Malaysia
}

While lockdown is implemented to contain the COVID-19 pandemic, child abuse cases have been underreported despite the contrary increase in the number of calls to social helplines. Therefore, this review highlights child abuse during the COVID19 pandemic as a reference for future interventions. To identify the prevalence of different types of child abuse (physical, psychological/emotional, sexual, and neglect) across several studies. To discuss the factors associated with increased child abuse during the COVID-19 pandemic. Electronic databases such as PubMed, Google Scholar and ScienceDirect were searched using terms such as "COVID-19", "child abuse" and "prevalence". Several articles were read and retrieved based on their relevance, and their findings are included in this literature review. There have been increments in all four types of child abuse based on several studies, mostly committed by parents. For physical abuse, one study reported an increase by up to two times in the number of child abuse cases seen at the Emergency Department during the pandemic compared to the previous year. Psychological abuse was the most prevalent of all the other forms of abuse, with one study reporting that $88.7 \%$ of the children involved faced some form of psychological aggression, such as getting shouted at by caretakers. Sexual abuse and child neglect were more prevalent in low-income countries as children are forced to partake in self-exploitation to ensure continuous resources during this pandemic. Several factors were involved that lead to this surge in child abuse cases, such as parental stress secondary to lockdown measures, financial issues, and increased exposure to abusers. There is an increase in incidences of child abuse during this pandemic, mostly due to social and financial restraints. Hence, this review is hoped to assist the stakeholders to come up with strategies to halt the escalation of child abuse. 


\title{
POSTER PRESENTATION
}

\section{ID17 PP006}

\section{Acute Pancreatitis in Post Total Hip Replacement}

\author{
Azman, Nur Dina ${ }^{1}$; Md Shariff, Mohd Shaffid ${ }^{1}$; Bain, Mohd Asha'ari ${ }^{1}$; Nazarallah, Mohd Hilmi ${ }^{1}$; Norazmi, Abu \\ Ubaidah Amir ${ }^{1}$
}

${ }^{1}$ Department of Orthopaedics and Rehabilitation, Universiti Sains Islam Malaysia, Negeri Sembilan, Malaysia

Total hip arthroplasty is known and accepted as a modality of treatment for severe avascular necrosis of hip. Despite its safety, there are still risk of developing systemic complications. Among major complications, gastrointestinal complications are less to occur compared to cardiovascular or respiratory. Up to this time being, less than five cases have been reported pertaining to acute pancreatitis as a complication following arthroplasty surgery. A 73-year-old lady with underlying diabetes mellitus, hypertension and hypercholesterolemia was diagnosed with left hip avascular necrosis. She underwent left total hip replacement under general anesthesia. The surgery was uneventful. However, post operatively she complained of abdominal discomfort associated with nausea and vomiting. She remained apyretic and tachycardic. Blood investigations showed significant raise in amylase. Ultrasound revealed multiple tiny gallbladder calculi. The diagnosis of acute pancreatitis was made. Postoperative complications of arthroplasty have been divided into systemic or local complications. In terms of systemic complications, gastrointestinal is accounted less in the list and most of it are cases of ileus, gastrointestinal bleed, and bowel obstruction. Acute pancreatitis is less common. A case has been reported on patient with multiple risk factors following bilateral simultaneous knee arthroplasty surgery. There is also a Japanese abstract referencing similar event in bilateral total knee arthroplasty without no risk factor present. Alcohol consumption and cholelithiasis accounts about $80 \%$ of risk factor for developing acute pancreatitis. However, in patient with cholelithiasis, the incidence to develop pancreatitis is less than 1\%. Propofol induced pancreatitis has also been reported in a nonabdominal surgery to a young nonalcoholic patient. The diagnosis of acute pancreatitis must be entertained in patient with risk factors underwent arthroplasty. Even though it is uncommon, early diagnosis can prompt crucial treatment. 


\section{POSTER PRESENTATION}

\section{ID18 PP007}

\section{Virtual Reality (VR) Locomotion in Increasing Young Adults Physical Activity Tendency}

Hasiff ${ }^{1}$, Kharmila A. $^{2}$

${ }^{1}$ Islamic Science University of Malaysia, Negeri Sembilan, Malaysia

Non-communicable disease especially Type-2 Diabetes Mellitus (T2DM) and obesity is a rising concern of almost every country in the world. According to National Health Morbidity Survey (NHMS), the prevalence of T2DM rise 4-fold and obesity rise to $280 \%$ during the 30 years period in Malaysia and it does not show any signs to stop. Among the leading factors that causing increase in obesity prevalence is reduction in physical activity, especially in the young adults. The reducing physical activity in young adults corresponds to the advancement of technology which provides easier access to social gaming and social media. This study describes the use of Virtual Reality (VR) locomotion, which integrates gaming into virtual world to increase physical activity among young adults. The main objective is to overview the viability and effectiveness of VR locomotion in increasing young adults' physical activity tendency in Malaysia. Literature review was conducted based on selected 11 papers under keyword "Obesity, Physical Activity, Virtual Reality, and Video Game". The main sources were on Pubmed, IEEE Xplore and Google Scholar. From those papers, 10 studies reported that VR can increase physical tendency of participants with high enjoyment level. Only 1 study reported that VR only increased the heartbeat in the participants but did not increase in energy expenditure. However, the study limitation is it did not involve rhythmic activity of large muscles but only quick shuffling as method of navigation. The result of VR locomotion as health intervention in other papers are very supportive to our notion. However, those studies are done outside Malaysia. Thus, a study should be conducted on young adults in Malaysia as representative of the Asian population. Conclusion: VR locomotion is on the right track to prove its effectiveness in tackling low physical activity tendency in young adults. 


\section{POSTER PRESENTATION}

\section{ID19 PP008}

\section{Artificial Intelligence Development in Making Diagnosis and Management Plan}

Hasiff $^{1}$, Arief $^{H} .^{1}$

${ }^{1}$ Islamic Science University of Malaysia, Negeri Sembilan, Malaysia

There are expanding use of electronic health record (EHRs) and public biomedical datasets that are growing exponentially like GenBank and caBig in other advancing countries such as United States. The advancement in this part of technology is a perfect set up for applications of computational and Artificial Intelligence (Al) techniques to disclose the fundamental patterns that can be used to predict initial diagnosis, management, minimize medical errors or cost and integrate up-to-date research into current practice amidst multiple major problems arise in the term of function and delivery in modern healthcare system. The main objective is to review the development and feasibility of $\mathrm{Al}$ in giving an accurate initial diagnosis and management plan with current technology. The literature review was based on selected 10 papers under keyword "Artificial Intelligence, Medicine, Diagnostic, and Management". The main sources of the literature reviews were on Pubmed, IEEE Xplore and Google Scholar. Out of the 10 papers, 6 papers support on how Al can detect pathology, illness and induce diagnosis. 2 papers highlight on role of Al being supportive medium to doctors while the other 2 papers spotlight on how Al can improve workflow and data management. In addition, the exponential pace of current technology advancement also acts as a booster to Al development in medical setting. However, 9 of the papers stressed on possible limitation of Al such as data privacy, data quality and biased information. Al in medical setting is moving in accelerating phase concurrent with the technology advancement. Al potentially can promise diagnosis, management, and other beneficial aspect both to the healthcare providers and healthcare consumers such as administrative task and improve documentation. Nevertheless, the potential of Al in medical setting is vast and the opportunity to explore it further should not be taken for granted. 


\section{POSTER PRESENTATION}

\section{ID20 PP009}

\section{Running Economy: Secret of Long-distance Running}

$\underline{\text { Zul I }}{ }^{1}$; Mohd Noor MT ${ }^{1}$

${ }^{1}$ Faculty of Medicine and Health Sciences, Universiti Sains Islam Malaysia (USIM), Negeri Sembilan, Malaysia

Running economy (RE) is defined as oxygen uptake that needed at a given submaximal velocity. Eliud Kipchoge became the first person in the history to complete a marathon in under two hours. Though the record was an unofficial, it is a significant milestone for the long-distance runners. The objectives were to discuss the running biomechanics and the physiological factors related to long-distance running, discuss the components of running economy and how it improves the performance of long-distance athletes and the factors that influence running economy. A literature review of published articles was conducted based on the objectives. Electronic databases such as PubMed, EBSCOhost and Google scholar were used. Type of studies include all primary studies such as Randomized controlled trial, Cohort studies, systematic reviews, and literature reviews. Excellent RE utilize minimal amount of oxygen compared to one with poor RE which using more oxygen in the same speed. Basically, the actual running velocity at lactate threshold, can be determined by running economy as it interacts with VO2max and lactate threshold. The factors that influence better running economy include strength training, plyometric training and endurance which were found in 6 research. Environmental, biomechanics and anthropometric factors were also found in many research to improve running economy. These factors help runners to run more efficiently. Running economy plays a major role in long-distance running other than higher VO2max and lactate threshold. Running economy shows how efficient the runners to use $\mathrm{O} 2$ during running and there is other several factors found by researchers which influence running economy. 


\title{
POSTER PRESENTATION
}

\section{ID22 PP011}

\section{Usable security in Electronic Health Record System: A review on the Saudi Arabian perspective}

\author{
Samina A. Khan ${ }^{1,2}$, Syaheerah L. Lutfi ${ }^{1}$ \\ ${ }^{1}$ School of Computer Sciences, Universiti Sains Malaysia, 11800 Minden, Penang, Malaysia \\ ${ }^{2}$ Medical Informatics and E-Learning Unit, Department of Medical Education, College of Medicine, King Saud \\ University, Riyadh, Saudi Arabia
}

The electronic health record (EHR) is one of the most prominent achievements of healthcare information technology, making it the fundamental building block of digital healthcare. With the increase in sensitive and personal data stored in the EHR system, the usability of the system and information security have become fundamental concerns. Experts are constantly developing techniques to improve security while maintaining usability. This is where usable security comes into play. More specifically, in the context of this review paper and in the field of healthcare, it is defined as how easy it is to use the security of the system. This review paper analyses the available evidence on the security and usability of healthcare software to derive recommendations for the Saudi healthcare sector. A review of the current literature was conducted using PubMed, Google Scholar, and the results were analyzed. Healthcare professionals work under time pressure and with limited resources. The need for efficient and usable digital tools that include security measures has become a necessity. A lack of security undermines the safety of the information stored in the system and ultimately jeopardizes patient safety. Unfortunately, due to the sensitive context of healthcare, there are no clear global guidelines for usable security. Usability research in other fields suggests that such research would need to be specific to a country or region with common cultural and linguistic characteristics. The impact of usability in EHR system security, and health care in general in general should be empirically investigated in future studies in hospitals from Saudi Arabian in the context of health care professionals. 


\section{POSTER PRESENTATION}

\section{ID25 PP012}

\section{Coding error of Otorhinolaryngology cases in 2020 at one of the University Hospitals in Malaysia according to International Classification of Diseases, Tenth Revision (ICD-10)}

Shah Nizamuddin, Muhammad Khairul Asraf ${ }^{1}$; Mohamad Yasim, Mohamad Helmi²; Azzeri, Amirah ${ }^{3}$; Sidek, Salawati ; Tengku Omar, Tengku Ahmad Shahrizal ${ }^{4}$; Dahlui, Maznah¹.

${ }^{1}$ Department of Social and Preventive Medicine, Faculty of Medicine, University of Malaya, Kuala Lumpur, Malaysia

${ }^{2}$ Department of Research, Development, and Innovation, Universiti Malaya Medical Centre, Kuala Lumpur, Malaysia

${ }^{3}$ Faculty of Medicine and Health Science, University Sains Islam Malaysia, Nilai, Negeri Sembilan, Malaysia ${ }^{4}$ Department of Otorhinolaryngology, Universiti Malaya Medical Centre, Kuala Lumpur, Malaysia

The otorhinolaryngology admissions are common at University Malaya Medical Centre (UMMC). Coding in the Casemix system is prone to error and coding error is crucial to be avoided for accuracy in assigning the Diagnosis Related Groups (DRGs) codes. This study aims to establish the prevalence and reasons behind the ICD-10 coding errors among otorhinolaryngology cases at UMMC. Data was retrieved from the hospital information system and after data cleaning, 106 data were available from otorhinolaryngology admission from January to November 2020. The data was then reviewed and matched with case-mix code. If there were discrepancies, two reviewers reviewed them together with the discharge summary of the electronic medical records and the case-mix system to find the correct ICD-10 code and the reason for the errors. The result showed there were 73 correct ICD-10 codes out of 106 data available (68.9\%). From the $31.1 \%$ errors, there were three types of errors in this study. The errors were errors in discharge summary $(16.0 \%)$, errors in extracting data $(10.4 \%)$, and errors in both discharge summary and data extraction (4.8\%). Coding errors are going to affect the accuracy of the diagnosis-Related Group (DRG) in the case-mix system and then, affect the cost of each DRG produced for each case. Further works need to be conducted to reduce the percentage of coding error by training the coders and training the medical officers. Thorough audits need to be done frequently to avoid increment percentage of errors and thus, loss of revenue from a miscalculation of budget can be reduced. 


\title{
POSTER PRESENTATION
}

\section{ID26 PP013}

\section{"Getting Unstuck" in A Disruptive Period of Medical Education: On Uncertainty and Vulnerability of Digital Learning and Moving Forward Through the Lens of Expansive Learning Cycle}

\author{
Abdul Rahman, Nur \\ ${ }^{1}$ Research Department of Primary Care and Population Health, Institute of Epidemiology and Health Care, \\ University College London, London, United Kingdom \\ ${ }^{2}$ Department of Primary Care Medicine, Faculty of Medicine and Health Sciences, Universiti Sains Islam \\ Malaysia, Nilai, Malaysia
}

The COVID-19 pandemic has increased the appetite for digital-learning like never. Medical education fraternity has responded as urgently as their clinical counterparts in finding feasible solutions to a crisis. However, despite the rapid reactions via elearning, students and faculties remain anxious with the prolonged disruption made worse by glaring digital divide among the stakeholders. Yet there seems to be no clear plans on how medical education in Malaysia is recovering from the initial shock. The aim of this paper is to outline a theory-driven methodology to overcome uncertainty and vulnerability of digitising medical education. Through an expansive learning cycle (Engëstrom, 1994), medical faculties can conduct root cause analysis for contradictions that impede current preclinical and clinical learning, and allow drawing of insights to create new objectives, models of activities and tools to overcome existing barriers. Local and specific implementation phases of new activity models using better tools will help to refine relevant processes and evaluate outcomes to the level where the activity model is part of a new norm until the next disruption prevails. The expansive learning cycle helps discover contradictions and causes of problems. It instigates a logical approach to solutions, through making visible data that already exists within each medical faculty. Its steps are repeatable. Its iterative process also facilitates faculty members to be more adaptive to change, to design agile solutions and to be future-ready. Getting unstuck amidst digital divide and rapid uptake of e-learning calls for systematic ways of problem solving. Medical faculties must not leave medical students' plight of uncertainty and vulnerability to chance. Expansive learning cycle is a proactive theory-driven method that could potentially overcome the disruption. This way, medical education will continue to inspire existing students and future generations to remain hopeful in the medical vocation. 


\title{
POSTER PRESENTATION
}

\section{ID28 PP014}

\section{Hodgkin Lymphoma with Gastric Invasion: A Case Report}

\author{
$\underline{\text { Azzahra Azhar }}{ }^{1,4,5}$, Abu Dzarr Abdullah ${ }^{2,3}$, Nor Aslina Abd Samat ${ }^{2,3}$, Nur Asma Sapiai ${ }^{3,4,}$ Faezahtul Arbaeyah \\ Hussain $^{1,4}$ \\ ${ }^{1}$ Department of Pathology, 2Department of Internal Medicine, ${ }^{3}$ Department of Radiology, School of Medical \\ Sciences, Health Campus Universiti Sains Malaysia, Kubang Kerian, Kelantan, Malaysia. \\ ${ }^{4}$ Hospital Universiti Sains Malaysia, Kubang Kerian, Kelantan, Malaysia. \\ ${ }^{5}$ Faculty of Medicine, Universiti Sultan Zainal Abidin, Kuala Terengganu, Terengganu, Malaysia.
}

Hodgkin lymphoma $(\mathrm{HL})$ commonly present with lymph nodes enlargement. $\mathrm{HL}$ also has a metastatic behavior like the other types of malignancy. Approximately up to $10 \%$ cases of lymphoma metastases through hematogenous spread involving gastrointestinal tract $(\mathrm{GI})$, lungs and others organ. We report a case of a-35-yearold female was diagnosed with nodular sclerosing Hodgkin lymphoma from left cervical lymph node in 2015. She had two cycles of chemotherapy before defaulted due to pregnancy. She represented in 2018 with two months history of epigastric pain. Radioimaging showed multiple abdominal lymph nodes enlargement with stomach infiltration. Oesophagogastroduodenoscopy revealed large and ulcerated mass with nodules at the gastric fundus. Tissue biopsy showed infiltration by Hodgkin lymphoma. She had disease progression and died due to lung infection before completing her fourth cycle of chemotherapy. Stomach is a rare site for HL metastasis. However, in this case, there is a possibility of tumour infiltration arising from the adjacent neoplastic lymph node. It is known that HL commonly seen in the lymph node. However, the infiltration of the tumour into adjacent or distal tissue should not be dismissed. A thorough histopathology including immunohistochemistry examination is recommended. 


\title{
POSTER PRESENTATION
}

\section{ID29 PP015}

\section{Dental icon- spreading oral health education through video making during the COVID-19 pandemic}

\author{
$\underline{\text { Radzniwan Rashid }}{ }^{1}$, Siti Zuriana Mohd Zamzuri², Cheng Lai Choo ${ }^{2}$ \\ ${ }^{1}$ Family Medicine Unit, Faculty of Medicine and Health Sciences, Universiti Sains Islam Malaysia, Nilai, \\ N.Sembilan, Malaysia \\ ${ }^{2}$ Oral Health Division, State Health Department, Federal Territory of Kuala Lumpur \& Putrajaya, Malaysia
}

Dental icon is an established entity formed by Oral Health Program Ministry of Health Malaysia in 2017 mainly to embrace oral health promotion and education among communities in Malaysia. Uniquely, the individual among dental icons is selected from the public and not from dental fraternity at all. During the pandemic, the usual face to face activities were impossible hence delivering knowledge by digital technology is a norm. Delivering education through video is an option and is widely practiced to ensure continuity of education especially among the communities. The objective of this paper is to delivers optimum oral health education via video making uploaded in social media platform during the pandemic Covid-19 while reducing the virus transmission via avoiding face-to-face interaction. Crucial topics related to oral health care and health were determined prior the video-making. The contents were extracted from well-prepared dental icon modules, Ministry of Health Malaysia's website and were reviewed by dental experts. The created videos then were uploaded on respective personal social media platform belonged to the dental icon. About 5 videos were created and published in a personal social media platform such as Facebook, Instagram and YouTube from May 2020 until July 2021. The topics included education on dental caries, basic oral health care techniques such as proper bruising of teeth, toothpaste, dental visits, the prevention towards oral cancer and when to get help. These videos obtained positive feedback from the netizen and some requested permission to be utilized for their children's oral health care education. Education is greatly impacted during the pandemic as face-to-face activities are avoided to reduce COVID-19 transmission. Nonetheless the advance in technology such delivering the education via video on the subject enables the teaching about oral health care to be continually provided despite the obstacle. 


\title{
POSTER PRESENTATION
}

\section{ID31 PP016}

\section{The Impact, Challenges, and the Consequences of COVID-19 on Non- Communicable Disease}

\author{
Zulfa Ghazali ${ }^{1}$; Mohd Radzniwan A. Rashid²; Sharifah Najwa Syed Mohamad²; Nuruliza Roslan ${ }^{3,4}$ \\ ${ }^{1}$ Faculty of Medicine and Health Science, Universiti Sains Islam Malaysia (USIM) \\ 2 Department of Primary Health Care, Faculty of Medicine and Health Science, Universiti Sains Islam Malaysia \\ (USIM) \\ ${ }^{3}$ Biochemistry Unit, Department of Medical Sciences 1, Faculty of Medicine and Health Science, Universiti \\ Sains Islam Malaysia (USIM) \\ ${ }^{4}$ Islamic Science Institute, Universiti Sains Islam Malaysia (USIM)
}

The emergence of COVID-19 has impacted the status of Non-Communicable Diseases (NCDs) and seen to affect patients' access to the healthcare system. This includes the transformation of human resources and medical attention more to the emergency response toward COVID-19 across the globe. In this review, we explored the challenges imposed by COVID-19 pandemic on stroke, ischaemic heart disease, hypertension, diabetes mellitus, cancers and respiratory disorders. To review the impact, challenges and the consequences of COVID-19 pandemic on the care of the NCDs globally. Related articles were reviewed and analysed from the search engine using from electronic databases: PubMed, Google Scholar, EZproxy, UptoDate from 2016 until 2021. The most searched keywords were "Non-Communicable Diseases" or "COVID-19" or "challenges" or "strategies" and "healthcare system". A total of 28 articles were reviewed. Most countries have implemented 'lockdown' to combat COVID-19 pandemic. However, the order has caused significant problems for patients with NCDs who are in dire need for regular follow-ups and consultation. The number of acute presentations and hospitalization related to NCD were reduced due to fear of contracting COVID-19 at the hospital. Extra precautionary steps were considerably observed. In addition, disease prevention and screening programmes for NCDs were decreased resultant from complying with lockdown order. Consequently, substantial disruptions of NCD care were noted. Nonetheless, current and future plans have been provided by the World Health Organization (WHO) to keep the NCD status at the optimum level of care. COVID-19 has disrupted the common practice of NCD handling worldwide, resulting in poor control in most NCDs. Utilisation of health care, the referral system and related administrative works are among the spectrum being severely disrupted. 


\section{POSTER PRESENTATION}

\section{ID32 PP017}

\section{Family Planning Uptake among Married Adults in Asia: A Systematic Review}

Mohamad Yusuff, Aza Sherin ${ }^{1}$; Zainuddin, Huzaimi ${ }^{1}$; Zainal, Adilah ${ }^{1}$; Ahmad Lutfi, Maryam ${ }^{1}$; Abdul Rahman, Shahir ${ }^{1}$; Zaidi, Abrar; Zakaria, Luqman Hakim ${ }^{1}$; Syed Ibrahim, Sharifah Nahidhah ${ }^{1}$; Mokhtar, Mastura ${ }^{1}$; Ariffin, Husna ${ }^{1}$; Ibrahim, Izuddin ${ }^{1}$; Azhar, Khaidir'; Samson, Waras; Azhar, Arisa Hanis ${ }^{1}$; Mohd Rani, Dzulkhairi ${ }^{1}$; Abu Baharin, Fakhri'; Mohamad Nor, Nadeeya 'Ayn ${ }^{1}$; Baharom, Nizam¹; T Mohd, Madeehah ${ }^{1}$; A Rahman, Zairina ${ }^{1}$; Jaafar, Mohd Hafiz ${ }^{1}$; Azzeri, Amirah ${ }^{1}$

${ }^{1}$ Public Health Unit, Department of Primary Health Care, Faculty of Medicine and Health Sciences, Universiti Sains Islam Malaysia

Family planning uptake (FPU) among countries in Asia are high even in low to high income countries. However, previous systematic reviews on FPU only cover certain geographical location, focused on certain gender, general marital status and more than 10 years ago. There are also several factors that are associated with the difference in FPU. This review aims to synthesize the available evidence on FPU and its associated factors among married adults in Asia. Quantitative and qualitative studies in Asia, published in English language between 2011 to 2021 among adults above 18 years old were considered. Methodological quality of included studies was assessed using standardized tools. From 8222 articles, 191 were selected for fulltext review and only 41 articles met the review eligibility criteria includes crosssectional, cohort and case control studies. A total of 22 of the articles discussed the modern and traditional methods of family planning while 17 articles only focused on the modern methods. Family planning uptake range from $21.0 \%$ to $83.1 \%$. The most common modern method of contraception used were contraceptive pills, condoms, and implants. The typical traditional method of family planning was rhythm and birth limitation. Female, older age group, high knowledge and attitude towards family planning, good spousal communications and living in an urban area were found to be associated with FPU. The utilization of family planning among Asians was moderate to good. Nevertheless, efforts to encourage the use of family planning should be strengthened to prevent any complications related to unplanned and unwanted pregnancies. 


\section{POSTER PRESENTATION}

\section{ID34 PP018}

\section{IR 4.0 - Additive Manufacturing (3D Printing) Emergency Devices}

Mohd Ifwat Mohd Ghazali ${ }^{1}$, Ahmad Syukran Baharuddin², Muhammad Syafiq Alauddin ${ }^{3}$, Sukor Romat, Muhammad Taufik Mohd Noor ${ }^{4}$

${ }^{1}$ SMART RG, Faculty Science and Technology, Universiti Sains Islam Malaysia (USIM), Negeri Sembilan, Malaysia ${ }^{2}$ Faculty of Syariah and Law, Universiti Sains Islam Malaysia (USIM), Bandar Baru Nilai, 71800 Nilai, Negeri Sembilan

${ }^{3}$ Faculty of Dentistry, Universiti Sains Islam Malaysia (USIM), Level 15, Tower B, Persiaran MPAJ, Jalan Pandan Utama, 55100, Kuala Lumpur

${ }^{4}$ Faculty of Medicine and Health Sciences, Universiti Sains Islam Malaysia (USIM), Negeri Sembilan, Malaysia

Industrial Revolution (IR) 4.0 is an integration of autonomous machinery with data analytical method for manufacturing industries. IR 4.0 provides a combination of technology such as robotic automation, simulation, cloud computing, internet of things, cybersecurity, system integration, augmented reality, big data, and 3D printing (additive manufacturing). Additive manufacturing among the technologies have become essential particularly during the COVID-19 pandemic. The objective of this paper is to discusses the role of IR 4.0 during the COVID-19 pandemic, discuss usage of additive manufacturing for emergency medical devices and the future prospect of the additive manufacturing for the future of medical devices. A literature review of published articles was conducted based on the objectives. Electronic databases such as PubMed, EBSCOhost and Google scholar were used. Type of studies include all primary studies such as randomized controlled trial, cohort studies, systematic review, and literature review. IR 4.0 have created a paradigm shift in the world of manufacturing. Additive manufacturing can customize and manufacture on the spot product which can be used instantly. This is more evident during the pandemic where several medical devices are manufactured using additive manufacturing technology. Devices such as ventilator valve, nasopharyngeal swabs, face shield, respiratory mask and hands-free handle devices are manufactured during COVID-19 pandemic. Additive manufacturing is an emerging technology that is useful for developing fast prototype product for emergency medical devices. With the current development of new materials, different printing technique and multiple functionalities for product design, additive manufacturing has opened new horizon in term of medical product manufacturing. 


\title{
POSTER PRESENTATION
}

\section{ID36 PP019}

\section{Initiative for Teleconsultation at Klinik Pakar Kesihatan USIM: Embarking on digital health during the COVID-19 Pandemic}

\author{
Syed Mohamad, Sharifah Najwa1,2; A. Rashid, Mohd Radzniwan¹,2; Iskandar Mirza, Azrul Azlan ${ }^{2,3}$ \\ ${ }^{1}$ Department of Primary Health Care, Faculty of Medicine and Health Sciences, Universiti Sains Islam Malaysia, Nilai, \\ Malaysia \\ ${ }^{2}$ Klinik Pakar Kesihatan USIM, Nilai, Malaysia. \\ ${ }^{3}$ Faculty of Economics and Muamalat, Universiti Sains Islam Malaysia, Nilai, Malaysia
}

Telemedicine is becoming a new norm in managing patients during the COVID-19 pandemic despite its first introduction in Malaysia in 1997. The advantages include ensuring contactless patient management thus avoiding the spread of the disease and provide an indirectly safe positive environment without compromising the usual patient care. There are many instances where the method has been applied to suit the needs of both the physician and patient. This case illustrates the challenges of how Klinik Pakar Kesihatan USIM (KPKU) embarked on an initiative of a branch of telemedicine, namely teleconsultation. KPKU is a corporate waqf concept clinic that was first established in April 2015. During the COVID-19 pandemic, an initiative was made to conduct teleconsultation. So far three cases have utilized the method since June 2021. These sessions were considered successful with need of internet connectivity enhancement. Other challenges include the establishment of policy, process and procedure, terms and conditions, ethical considerations, choosing a suitable platform, strong internet connection, determining target patients, consultation effectiveness, record-keeping, client-appointment and payment system and medication courier service. Benefits of teleconsultation include reduced exposure to infectious disease, sense of comfort for both the treating physician and client, cost-effectiveness, and ease of appointment arrangement via online platform. Limitations of teleconsultation are the lack of "true" doctor-patient relationship, lack of clinical assessment and only "stable" diseases are considered safe to undergo a teleconsultation. Careful scrutinization and discussion are crucial in decision making and appropriate selection of the mentioned points above. Providing teleconsultation via an online platform to discuss clients' concerns is one way that healthcare service can be delivered in a safe and comfortable manner to the end user. However, this contemporary method of telemedicine is not without challenges and requires detailed risk assessment and suitability of the healthcare setting. 


\title{
POSTER PRESENTATION
}

\section{ID37 PP020}

\section{Direct Medical Cost of Stroke and The Cost-Effectiveness of Direct Oral Anticoagulants Among Atrial Fibrillation-Related Stroke Patients in Malaysia}

\author{
Norliana Masbah ${ }^{1}$; Siti Norain Azahar ${ }^{1}$; Saperi Sulong ${ }^{2}$; Wan Asyraf Wan Zaidi ${ }^{3}$; Kamisah Yusof ${ }^{1}$; Norliza \\ Muhammad'. \\ ${ }^{1}$ Department of Pharmacology, Faculty of Medicine, Universiti Kebangsaan Malaysia, Kuala Lumpur, Malaysia \\ ${ }^{2}$ Department of Community Health, Faculty of Medicine, Universiti Kebangsaan Malaysia, Kuala Lumpur, \\ Malaysia \\ ${ }^{3}$ Department of Internal Medicine, Faculty of Medicine, Universiti Kebangsaan Malaysia, Kuala Lumpur, \\ Malaysia
}

Stroke has significant direct medical costs and direct oral anticoagulants (DOACs) are better alternatives to warfarin for stroke prevention in atrial fibrillation (AF). Data on the direct medical costs of AF-related stroke and the cost-effectiveness of DOACs in Malaysia is scarce. This study was conducted to determine the direct medical costs of stroke, and AF stroke in a tertiary hospital in Malaysia. The costeffectiveness of DOACs compared with warfarin among stroke patients was also determined. This is a single-centre, retrospective study utilising in-patient data from Case Mix Unit of Universiti Kebangsaan Malaysia Medical Centre (UKMMC) between 2011 to 2018. Direct medical costs of stroke from the healthcare provider's perspectives were determined using a top-down costing approach and factors associated with costs were identified. Incremental cost effectiveness ratio (ICER) was calculated to compare the cost-effectiveness between DOACS and warfarin. The direct medical cost of stroke was MYR 11,669,414.83 ( $\mathrm{n}=3689)$. Median cost per episode of stroke care was MYR 2,269.79 (IQR 2,269.79 -2,924.96). AF-related stroke cases had higher median cost of MYR 2,839.73 (IQR 2269.79-3,101.52) and longer LOS of $6 \pm 4.2$ days. Regression analysis showed that stroke type (AF versus non-AF stroke) $(p=0.013)$, stroke severity $(p=0.01)$ and discharge status $(p<0.001)$ significantly influenced stroke costs. DOACs were cost-effective compared to warfarin with an ICER of MYR 19.25. Direct medical cost of stroke is substantial, with AF-stroke having a higher median cost per stroke care. DOACs were cost effective in the treatment of AF-related stroke in UKMMC. 


\section{POSTER PRESENTATION}

\section{ID38 PP021}

\section{Magnetic Resonance Imaging Study as Preferred Method in Diagnosing Morel-Lavallée Lesion}

Azman, Nur Dina ${ }^{1}$; Md Shariff, Mohd Shaffid ${ }^{1}$; Bain, Mohd Asha'ari ${ }^{1}$; Nazarallah, Mohd Hilmi ${ }^{1}$; Norazmi, Abu Ubaidah Amir

${ }^{1}$ Department of Orthopaedics and Rehabilitation, Universiti Sains Islam Malaysia, Negeri Sembilan, Malaysia

Morel-Lavallée lesion is a closed degloving injuries occurring deep to subcutaneous plane due to disruption of capillaries. It is most found in proximity to bony prominences. Magnetic resonance imaging (MRI) is the gold standard in the evaluation of Morel-Lavallée lesion. Delay in diagnosis or missed lesion will lead to infection or skin necrosis. We reported a case of 82 -year-old gentleman, with underlying hypertension presented with left hip swelling for past 1 year after a motor vehicle accident. It was worsening in size and associated with intermittent pain with no fever. Examination revealed single diffuse benign mass extended from gluteal region to proximal left thigh with healed bruises. Clinically, there was no sign of inflammation and infection. We proceeded with MRI due to recurrent benign swelling of the hip with no signs of infection. It was reported as presence of welldefined large multiloculated multiseptated heterogeneous collection located superficial to muscles of left hip and upper thigh. We concluded it as Morel- Lavallée lesion and unlikely tumour. Histopathological investigation confirmed the diagnosis. MRI is the imaging modality of choice which is specific and sensitive. From the MRI the lesions are well defined oval, fusiform, or crescentic and may have tapering margins that fuse with adjacent fascial planes. Lesions also may show fluid-fluid levels, septations, and variable internal signal intensity dependent on the concentration of hemolymphatic fluid and the acuity of the lesion. Lesions may have no capsule, a partial capsule, or complete capsule. Capsules are hypointense on all pulse sequences related to fibrous tissue and/or blood-by-products. MRI if being utilised appropriately will be able to confirm the diagnosis of recurrent soft tissue swelling to rule out infection and tumour. Early diagnosis will prevent complications and expedite management. 


\section{POSTER PRESENTATION}

\section{ID39 PP022}

\section{Dementia Prevention: Food, Exercise and Music}

Wan Muhammad Najib, Wan Muhammad Nazdmi ${ }^{1}$; Solehan, Hana Maizuliana ${ }^{2}$

${ }^{1}$ Faculty of Medicine and Health Sciences, Universiti Sains Islam Malaysia (USIM), Nilai, Negeri Sembilan, Malaysia

2Internal Medicine, Medical-Based Department, Faculty of Medicine and Health Sciences, Universiti Sains Islam Malaysia (USIM), Nilai, Negeri Sembilan, Malaysia

The cases of dementia among the Malaysian elderlies are increasing, as its prevalence in 2020 and 2050 is estimated to be $0.126 \%$ and $0.454 \%$, respectively. In the impossibility of developing any dementia treatment by 2025 , risk avoidance is the most effective way to prevent onset thus reducing the number of new cases. The aim of this article is to review the potential prevention of dementia which includes food, exercise and music. This is a comprehensive literature review, in which the relevant articles were sourced from electronic database such as Google Scholar and PubMed. The searched keywords were "dementia", "memory loss", "memory impairment", "cognitive impairment", “exercise”, "physical activities", "music" and various food types. The original articles were obtained, reviewed and important information were retrieved by all authors. Food such as polyphenolic compounds particularly resveratrol act as protective benefits on cognitive function. A dietary pattern consisting of high intakes of soybeans and soybean products, vegetables, algae, milk or dairy products, and low intakes of rice was linked to a lower risk of dementia. Those who had at least one fish meal a week have a $60 \%$ lower chance of Alzheimer's disease than someone who ate fish only sometimes or never. Exercise lowers the developing of dementia by eventually reducing the cardiovascular risk. Music-induced stimulation has been shown to improve cognitive function and arousal levels can affect memory via attention pathways. In conclusion, certain food such as grape juice, milk or dairy products and food containing omega3 fatty acids have certain role in dementia prevention. Exercise has a positive impact on brain neuroplasticity and resistance to brain ageing and neurodegeneration. Last, but not least, music does prevent dementia as music has been found to stimulate areas of the brain related to emotion, reward and decision-making benefit. 


\title{
POSTER PRESENTATION
}

\section{ID40 PP023}

\section{Telemedicine during the COVID-19 Pandemic: A Case Series of Our Experience}

\author{
Arief Hamid $^{1}$; Hana Maizuliana Solehan ${ }^{2}$
}

${ }^{1}$ Department of Surgery, Faculty of Medicine, University Sains Islam Malaysia, Nilai, Malaysia

${ }^{2}$ Department of Medicine, Faculty of Medicine, University Sains Islam Malaysia, Nilai, Malaysia

Telemedicine allows patients to connect to their doctors despite the travel restriction during the Movement Control Order (MCO) and reduce the risk of hospital acquired COVID-19 infection. We report non-COVID-19 cases that were successfully treated and followed up without having the need for physical presence at the hospital or clinic, using WhatsApp application and telephone consultation. Case 1: A 73-year-old Chinese lady with underlying hypertension and diabetes mellitus, presented with right hemiplegia and slurred speech was admitted for acute ischemic stroke. Initial blood pressure and blood glucose level were fluctuating. A telephone consultation was made two weeks after discharge. Home-monitoring blood pressure was lowish (100-110/50-60 $\mathrm{mmHg}$ ) and glucometer reading was $4-6 \mathrm{mmol} / \mathrm{L}$, thus subcutaneous actrapid and amlodipine were adjusted accordingly. Case 2: A 12-yearold Chinese boy was presented with a right plantar wart for 6 months. The initial consultation was performed via WhatsApp messenger by the parents. Information and photographs of the wart were obtained through digital consultation. He underwent wart excision as a daycare procedure 1 week later. Wound care instructions and all follow-ups post operation were made through WhatsApp messenger until full recovery. Case 3: A 70-year-old Malay lady successfully underwent elective laparoscopic cholecystectomy for gallbladder stone and was discharged on day- 2 post-operation. On day 5 , she developed a wound breakdown at her umbilicus. Consultations via WhatsApp messenger noted slough with serous discharge seen. Advice and technique of wound care were given to the patient's daughter. Regular digital wound reviews were made until complete wound heal, onemonth post-operation. Telemedicine use during COVID-19 pandemic helps to provide access to care, reduces hospital's congestion and provides safety to both patients and healthcare workers. Telemedicine should not reduce the provision of care given to the remote patient despite not being able to fully assess the patient's condition. 


\title{
POSTER PRESENTATION
}

\section{ID41 PP024}

\section{Occupational Psychological Well-Being Among Emergency Medical Service Ambulance Driver in Hospital Kuala Lumpur}

$\underline{\text { Rosdi Zainol }}{ }^{1}$, Shuib Rambat ${ }^{2}$, Norazlinda Saad $^{3}$

\author{
1 Unit Neurophysiology, Neurology Department, Hospital Kuala Lumpur \\ ${ }^{2}$ Malaysian-Japan International Institute of Technology (MJIIT), Universiti Teknologi Malaysia, Kuala Lumpur, \\ Malaysia \\ ${ }^{3}$ College of Arts and Sciences, Universiti Utara Malaysia, Sintok, Malaysia
}

Mitigation measures of occupational psychological issues among Emergency Medical Service (EMS) ambulance driver is the most crucial field of studies because it engages the safety and the life of a rescuer team members, a survivor or patient and the public. The objectives of this paper were to measures the self-reported occupational psychological issues of an EMS ambulance driver in the premier Hospital Kuala Lumpur (HKL), Malaysia. A cross-sectional and descriptive study using a target population sampling with 31 respondents was conducted using self-fill-in questionnaire of Perceived Stress Scale (PSS), Chalder Fatigue Scale (CFS) and Epworth Sleepiness Scale (ESS). Occupational psychological risk measures among 31 respondents for the PSS show 24 (78\%) were in the moderate category while one respondent having high PSS and 6 (19\%) respondents are in low PSS. The CFS found a total of $24(77 \%)$ respondents are under 'caseness' while only $7(23 \%)$ are under 'non-caseness'. While the ESS indicates that $20(65 \%)$ respondents having an excessive sleepiness during daytime while 11 (35\%) are free from sleepiness during daytime. Pearson coefficient correlation between fatigue and sleepiness is 0.651 , while between stress and fatigue is 0.563, and sleepiness and stress have a coefficient correlation of 0.429 . All three hypotheses have significant relationship. Prevalence of near-miss accident is relatively high (74\%) and fell asleep during driving also significant (52\%). Ambulance driver is at risk of experiencing occupational psychological symptoms. This finding highlights the importance of occupational psychological screening to combat the development of adverse health consequences. 


\title{
POSTER PRESENTATION
}

\section{ID43 PP025}

\section{Crescendo Transient Ischemic Attack' - An Uncommon Presentation of a Very Common Disease: A Case Report on Capsular Warning Syndrome Which Was Reviewed Regularly Via Teleconsultation}

\author{
Payus, Alvin Oliver ${ }^{1}$; Ibrahim, Azliza ${ }^{2}$; Wan Yahya, Wan Nur Nafisah ${ }^{3}$; Azman Ali, Raymond ${ }^{4}$ \\ ${ }^{1}$ Faculty of Medicine and Health Science, Universiti Malaysia Sabah (UMS), Jalan UMS, 88400 Kota Kinabalu, \\ Sabah, Malaysia \\ ${ }^{2}$ Department of Neurology, Hospital Pengajar Universiti Putra Malaysia, Persiaran Mardi - UPM, 43400 \\ Serdang, Selangor, Malaysia \\ ${ }^{3}$ Department of Internal Medicine, Universiti Kebangsaan Malaysia Medical Centre (UKMMC), Jalan Yaacob \\ Latif, 56000 Cheras, Kuala Lumpur, Malaysia \\ ${ }^{4}$ Department of medicine, Faculty of Medicine, Universiti Teknologi MARA, Jalan Hospital, 47000 Sungai \\ Buloh, Selangor, Malaysia
}

Capsular warning syndrome (CWS) is a rare presentation of transient ischemic attack (TIA), which described as recurrent episodes of motor and/or sensory deficits which typically sparring the cortical function. It has a significant risk to progress into a massive stroke with permanent disability, which makes it very important to be recognized early. A 60-year-old gentleman with no known medical illness presented with eight episodes of transient ischemic attack within the span of 24 hours. He was not subjected to thrombolysis at time of presentation because it was outside the window period of 4.5 hours and has fully recovered after each episode. Computed tomographic angiography of the brain was done and shows small well defined hypodensities at the body of right caudate nucleus. The major intracranial arteries have normal caliber and there is no filling defect to suggest thrombosis over the vertebrobasilar artery. He was treated with double antiplatelet for 21 days, then single antiplatelet for life. He was well upon regular follow up via teleconsultation every three months. CWS is a term to coin multiple episodes of stereotyped TIA which usually occur in the proximity of each other in time. CWS is rare, where the incidence was only 1.5 to $4.5 \%$ of TIA. However, it is particularly important because it has a high risk of developing massive ischemic stroke with a permanent neurological deficit. Therefore, dual antiplatelet for 21 days is recommended to prevent the development of full-blown stroke. The patient also required close follow up via teleconsultation. CWS is a rare spectrum of TIA which has high tendency to develop a full-blown stroke. Therefore, regular close clinic review via teleconsultation is important to follow up on the patient's condition. 


\title{
POSTER PRESENTATION
}

\section{ID44 PP026}

\section{An Uncommon Side Effect from Eating Durian Fruit - A Case Report on Life-Threatening Hyperkalaemia After Eating Durian Fruit and Follow Up Via Teleconsultation for Regular Review}

\author{
Payus, Alvin'; Sat Lin, Liew'; Azman Ali, Raymond ${ }^{2}$ \\ ${ }^{1}$ Faculty of Medicine and Health Science, Universiti Malaysia Sabah (UMS), Jalan UMS, 88400 Kota Kinabalu, \\ Sabah, Malaysia \\ ${ }^{2}$ Department of medicine, Faculty of Medicine, Universiti Teknologi MARA, Jalan Hospital, 47000 Sungai \\ Buloh, Selangor, Malaysia
}

Hyperkalemia is a condition of high serum potassium that occurs because of increased intake, or reduced clearance, or both. In a severe condition, hyperkalemia is a life-threatening medical condition that is fatal especially if recognized late and left untreated. There are many causes of hyperkalemia. However, eating durian fruit in the background of impaired kidney function is a very rare occurrence. A 79-yearold lady with hypertension, dyslipidemia and diabetes mellitus presented with a lifethreatening hyperkalemia with the potassium of $8.1 \mathrm{mmol} / \mathrm{L}$ because of eating large amount of durian fruit. She had a preceding history of multiple episodes of watery diarrhea for the past one week due to acute gastroenteritis which has just resolved but has led her to develop acute kidney injury. She was successfully treated with aggressive rehydration and potassium lowering therapy and was discharged well. Durian is a popular fruit in southeast Asia and named as the 'king of fruit' by the local people. It is rich in potassium where $100 \mathrm{gm}$ of durian flesh contain $436 \mathrm{mg}$ potassium. In a healthy individual, eating this fruit will not cause any danger as the body is capable to excrete the excessive potassium through the kidneys. However, when the kidney function is impaired, this feedback mechanism is lost. In our patient, her kidney function was impaired because of the recent episode of infective watery diarrhea. Therefore, she was unable to remove the excess potassium intake from the consumption of huge amount of durian fruit. Fortunately, she was managed promptly and discharged well. She was followed up regularly via clinic teleconsultation to monitor her symptoms. Consuming a large amount of durian fruit in the background of impaired kidney function can lead to a life-threatening hyperkalemia. A prompt management is vital to prevent sudden cardiac arrest. 


\section{POSTER PRESENTATION}

\section{ID45 PP027}

\section{Recommendation for Improvement in Malaysia's COVID-19 Vaccine Program: An Online Nationwide Qualitative Survey}

Hana Maizuliana Solehan ${ }^{1}$, Nurul Azmawati Mohamed ${ }^{1}$, Mohd Dzulkhairi Mohd Rani ${ }^{1}$, Muslimah Ithnin ${ }^{1}$, Ilina Isahak $^{1}$

${ }^{1}$ Faculty of Medicine and Health Sciences, Universiti Sains Islam Malaysia, Nilai, Negeri Sembilan

Maintaining high levels of vaccinations is important for public health success in any vaccination planning. Success requires a better understanding of the public's perceptions of diseases and consequent decisions about vaccinations. However, only a few studies have considered this from the public perspective on any immunisation program, particularly for the recently developed COVID-19 vaccine. This study aims to gather exploratory qualitative data on public opinion in regard to the Malaysian Government's COVID-19 immunization campaigns. This cross-sectional study was done using an online platform. An open-ended questionnaire using Google Form was distributed between 2 nd and 17th December 2020 and deactivated when saturation was reached. The responses were analysed using qualitative thematic analysis on a Microsoft Excel spreadsheet. Six hundred and sixty-one participants responded to this online survey. Four themes emerged from the thematic analysis on suggestions for the National COVID-19 Immunisation Program. The followings are the main themes (with its most mentioned subthemes): i) Vaccine administration policy (compulsory, available and accessible to all); ii) Dissemination of vaccine information (effective communication, more clinical trial data and addressing the antivaccine groups); iii) Other recommendations for the government (vaccine safety guarantee, external monitoring bodies, MySejahtera improvement); and iv) Local vaccine research and the use of alternative medicine. Participants believed that all these are essential in increasing their trust and uptake for the government's National COVID-19 Immunisation Program. This study highlights the suggestion made by the public for the Malaysian government. These findings will help the authorities improve the current intervention strategies to further accelerate the vaccine uptake among Malaysians, thus achieving herd immunity to combat the COVID-19 pandemic in Malaysia. 


\section{POSTER PRESENTATION}

\section{ID47 PP028}

\section{Mini Audit on Serum Procalcitonin Request by Using Data Mining}

Badaruddin, Izzatul Aliaa ${ }^{1}$; Md Mansor, Munirah'; Wan Shuaib; Wan M Azfar ${ }^{1}$; Ab Rahim, Siti Nadirah ${ }^{2}$; Nasuruddin; Dian Nasriana ${ }^{1}$.

${ }^{1}$ Department of Pathology, Faculty of Medicine, National University of Malaysia, Kuala Lumpur, Malaysia ${ }^{2}$ Department of Pathology, National Defense University of Malaysia, Kuala Lumpur, Malaysia

Serum procalcitonin is an established sepsis monitoring marker. In the current pandemic situation, serum procalcitonin has emerged as a prognostic marker in COVID-19 disease. Due to its specificity and sensitivity, serum procalcitonin request has increased in demand for research and clinical purposes, including inappropriate request and test abuse. We aimed to identify the locations of high workload for serum procalcitonin, revise the clinical justification for the request and observe the request trend throughout the audit period. An abrupt increase in the number of requests was identified, causing a shortage of reagents' stock for serum procalcitonin. An internal meeting was conducted to identify the causes. Three weeks of data for serum procalcitonin requests were extracted from the integrated laboratory management system (ILMS) and analysed using Microsoft Excel. The trends of the number of requests throughout the preaudit period were observed daily and in seven-day moving averages. The specialist in-charged of wards identified for the inappropriate request were approached via phone call, which resulted in an agreement for a stricter serum procalcitonin request. Three weeks of post-audit data were extracted and analysed, like the preaudit period. Results reviewed and concluded. A total of 971 requests were analysed, showing a $27 \%$ decrease in the number of requests and a $20 \%$ increase in the number of rejected requests postaudit. Higher workloads of serum procalcitonin were identified to be contributed from COVID-19-related units with few inappropriate test requests identified from few locations. The seven-day moving averages showed a decrease in request numbers for the initial ten days, but the trend increased afterwards, confounded by the surge of COVID-19 cases admitted to our institution. Continuous education and good communication between pathologists and clinicians are paramount to maintain continuous improvement of test service and ensuring judicious use of limited test resources. 


\section{POSTER PRESENTATION}

\section{ID49 PP030}

\section{Knowledge, Attitude and Perceived Confidence of USIM Dental Graduates Towards Management of Medical Emergencies}

Ahmad Tarmidzi, Nor Azura ${ }^{1}$; Mohamed Ramli, Nalisha ${ }^{1}$; Amran, Norashikin ${ }^{1}$; Norazmi, Husna Najihah²; Mohd Arifin, Nur Saniah ${ }^{2}$

${ }^{1}$ Department of Oral \& Maxillofacial Surgery, medicine and Pathology, Faculty of Dentistry, Universiti Sains Islam Malaysia, Kuala Lumpur

${ }^{2}$ Final Year Student, Faculty of Dentitsry, Universiti Sains Islam Malaysia, Kuala Lumpur

Medical emergencies can be alarming to any clinicians, but these situations can be managed well if proper preparation has been made. The objectives of this paper were to (1) evaluate USIM dental graduate's knowledge in dealing with medical emergencies in dental practice (2) assess perceived level of competency of USIM dental graduate's in dealing with medical emergencies in dental practice and (3) determine the need for further improvement in medical emergencies undergraduate training. A standardised questionnaire form consisting of demographic data, experience, knowledge, and perceived level of competency of dental practitioner in dealing with medical emergencies. 123 respondents answered the online questionnaire. More than half of them had experienced medical emergencies during dental treatment. All of them answered correctly on management of hypoglycemia but only $44.7 \%$ answered correctly on management of unresponsive patient and $65 \%$ answered correctly on position of syncope patient. Most of the respondents were not confident in performing intravenous medication (46.3\%), handling foreign body aspiration (24.4\%) and using AED (38.2\%). This study can be used as a guidance for further improvement in medical emergency training for undergraduates to enhance the proficiency level of USIM dental graduates. 


\section{POSTER PRESENTATION}

\section{ID50 PP031}

\section{Clinical Features and Outcome of ST-Elevation Myocardial Infarction (STEMI) in the Young Population at a Non-PCI Capable Hospital}

Amran, Norashikin ${ }^{1}$; Low, Wan Chia², Sia, Koon Ket ${ }^{2}$.

${ }^{1}$ Department of Oral \& Maxillofacial Surgery, Medicine and Pathology, Faculty of Dentistry, Universiti Sains Islam Malaysia, Kuala Lumpur

${ }^{2}$ Department of Internal Medicine, Hospital Ampang.

Coronary artery disease is one of the leading causes of deaths globally. The Malaysian National Cardiovascular Disease database (NCVD) stated that among all the patients with STEMI, the mean age of admissions was 56.0 years with $30.7 \%$ under 50 years old with $89.6 \%$ of patients are male. Our objective was to assess the prevalence, demographic distributions, and risk factors of STEMI in patients aged 45 years and less in our population. This is a retrospective study of all young patients aged 45 years old and below, admitted with STEMI to the Coronary Care Unit (CCU), Hospital Ampang from 1st January till 31st December 2019. There were 195 patients admitted to Hospital Ampang with STEMI with 50 patients (25.62\%) were below 45 years old in 2019. $42 \%$ of them were Malay, $18 \%$ were Indian, $10 \%$ were Chinese and $30 \%$ were various other nationalities. Among these patients, a significant proportion (92\%) were male and $8 \%$ were female. The median age was 40.5 and average age 38.9 years old. Majority of the patients (76\%) received thrombolysis treatment and $14 \%$ of the patients received percutaneous coronary intervention $(\mathrm{PCl})$. The rest were not given any urgent reperfusion treatment due to their late presentation to hospital. Two of the patients (4\%) required readmission within 30 days with the reason for readmission was acute heart failure. One of these patients eventually succumbed to his condition 4 months following the STEMI. Risk factors in this group were smoking $(62 \%)$, hypertension $(36 \%)$, diabetes mellitus $(28 \%)$, obesity $(16 \%)$, dyslipidaemia (14\%), history of ischaemic heart disease (14\%) and family history (6\%). A large proportion of the patients (54\%) have 2 or more risk factors. STEMI in young patients in our centre occurred most in males and smoking was the most common risk factor. 


\title{
POSTER PRESENTATION
}

\section{ID51 PP032}

\section{Malignant Melanoma of The Nose: To Manage the Rarity}

\author{
Ghazali, Liyana ${ }^{1}$; Mohd Noor, Rosli²; Ramli, Ramiza Ramza ${ }^{1}$; Yunus, Nor Hayati ${ }^{3}$
}

${ }^{1}$ Department of Otorhinolaryngology-Head Neck Surgery, School of Medical Sciences Universiti Sains Malaysia Health Campus, Kelantan, Malaysia

${ }^{2}$ Department of Otorhinolaryngology, Hospital Raja Perempuan Zainab II, Kelantan, Malaysia

${ }^{3}$ Department of Pathology, Hospital Raja Perempuan Zainab II, Kelantan, Malaysia

Malignant melanoma of nose is an extremely rare and lethal tumour with unknown etiology and poor prognosis. In the early stage, it usually presented with non-specific symptoms that include nasal obstruction, epistaxis and rhinorrhea masking the disease till it's at the advanced stages. Therefore, surgical intervention is complicated and yields poor outcome. A 54-year-old female presented with left nasal blockage and left epistaxis for about one month. She also complained of left nasal discharge and hyposmia. Nasal endoscopy examination of the left nostril showed a bluish to black rounded mass originating from the lateral wall of the nose measuring about $2 \mathrm{~cm} \times 1 \mathrm{~cm}$ which bleeds upon manipulation. Biopsy taken from the mass was suggestive of malignant melanoma. She was counselled for complete excision of the mass; however, she did not agree for further surgical intervention, thus she was referred to radiation therapy. During her follow up, she showed marked improvement of the symptoms after completed 20 sessions of radiotherapy. Repeated computed tomography scan revealed reducing size of the mass with no distant metastasis. This case illustrates the management of malignant nasal melanoma of the nose. The prognosis of the disease depends on the stage of presentation. Due to its unspecific symptoms an early diagnosis is a challenge. Surgical resection followed by radiotherapy is the treatment of choice for localized tumour but radiotherapy alone has shown some promising outcome. Early referral to oncology may contribute for good outcome. However, local recurrences and distant metastasis may occur after curative therapy accounting for its bad prognosis. Thus, long term follow up is required. 


\section{POSTER PRESENTATION}

\section{ID53 PP034}

\section{Estimating Current Active Cases of COVID -19 Post School Re-Opening in Malaysia Using SIR (Susceptible - Infected - Removed) Model}

Azrul Azim Mohd Yunus ${ }^{1}$, Shahrina Ismail' ${ }^{1}$, Muhammad Safwan Ibrahim ${ }^{1}$, Arif Asraf Mohd Yunus ${ }^{1}$.

${ }^{1}$ Department of Financial Mathematics, Faculty of Science and Technology, Universiti Sains Islam Malaysia, Negeri Sembilan, Malaysia.

There have been 1,061,476 infections and 8,551 coronavirus-related deaths reported in the country since the pandemic began as of 29th July 2021. This drastic increase in numbers has been alarming everyone. Malaysia has successfully handled the first and second waves of the covid-19 cases. However, after 1st April 2021, Malaysia has seen an increase in active cases. In this paper, we use the SIR model (Susceptible Infected - Removed) to estimate the active cases in Malaysia. We forecast the active case of COVID-19 case reach its peak on 26th of May 2021 with infection rate $=0.12$, $\mathrm{RO}=1.2$. Our proposed model was found to be successful in predicting peaks and the duration of the outbreaks. Besides that, to reduce future outbreaks, the number of susceptible can be reduced via vaccinations. Therefore, with proper planning and execution, the inoculation rates in Malaysia proved to be successful, where the impact of mass vaccination would be seen in the very near future. 


\title{
POSTER PRESENTATION
}

\section{ID54 PP035}

\section{Oral Contraceptive Pill (OCP) and risk of cancer: a review}

\author{
Anuar, Balkhis ${ }^{1}$; Abdul Hamid, Nazefah ${ }^{1}$
}

${ }^{1}$ Faculty of Medicine and Health Sciences, Universiti Sains Islam Malaysia, Negeri Sembilan, Malaysia

Oral contraceptive pill (OCP) is a form of birth control that contains a combination of estrogen and progestin, progesterone alone, and a continuous or prolonged use tablet. OCP-related cancer has been widely reported, and a recent study found that the OCP does not increase all OCP-related cancer, but it does show a reduction in some cancer risks. The objective of this paper is to reviews the relation of OCPrelated cancers (breast, cervical, endometrial, and ovarian cancer). Literature review was conducted using PubMed, Science Direct, ProQuest and Google Scholar. The keywords used were 'oral contraceptive pills' AND 'breast cancer' AND 'cervical cancer' AND 'ovarian cancer' AND 'endometrial cancer'. The search was limited to English language and the full paper was analysed accordingly. A study reported that the cancer risk increased in women who had used the OCP compared to women that were not ever exposed to the OCP. This is supported by a later study in 2010 that showed a slight increase in breast cancer risk in the participant that used OCP. An analysis of 8 studies conducted by the International Agency for Research on Cancer, as well as a systematic review and recent major epidemiological links that strongly suggest an increased risk of cervical cancer, but only for women who had been using it for at least 5 years and had persistent human papillomavirus infections of the cervix. In contrast, women that used the OCP in the past have a lower risk of ovarian cancer compared to the women that never used OCP. The OCP has been found to reduce the risk of cancer in the context of endometrial cancer. The risk was reduced by at least 30\%, and the protection lasted for many years after the pills were stopped. Despite there are studies reporting the increased risk of cancer with the OCP use, these issues remain controversial. However, the health benefits of OCP far exceed the cancer risks, particularly in developing countries where the risk of maternal mortality is high. Thus, consideration of this benefit can be made along with careful consideration of the other known risks and benefits of OCP use. 


\section{POSTER PRESENTATION}

\section{ID55 PP036}

\section{The Impact of COVID-19 on Perinatal Outcome: A Literature Review}

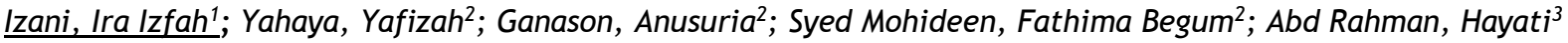

${ }^{1}$ Faculty of Medicine and Health Sciences, Universiti Sains Islam Malaysia, Bandar Baru Nilai, 71800, Nilai, Negeri Sembilan, Malaysia

${ }^{2}$ Family Medicine Unit, Faculty of Medicine and Health Sciences, Universiti Sains Islam Malaysia, Bandar Baru Nilai, 71800, Nilai, Negeri Sembilan, Malaysia

${ }^{3}$ Pathology Unit, Department of Medical Science II, Faculty of Medicine and Health Sciences, Universiti Sains Islam Malaysia, Bandar Baru Nilai, 71800, Nilai, Negeri Sembilan, Malaysia

Virus COVID-19 is highly infectious. Pregnant mothers are in a high-risk group when being infected. In United States, there were 94,519 COVID-19 cases involving pregnant mothers with 103 deaths among them. However, evidence of possible vertical transmission is scarce. Several published papers investigated the perinatal outcome in terms of its direct effect on pregnancy, probability of vertical transmission and effect of breastfeeding and neonatal contact. The objective is to review recent literatures on the impact of COVID-19 on perinatal outcomes. Specifically, this study aims to clarify three main concerns: the direct effect of COVID-19 on pregnancy, the possibility of vertical transmission of COVID-19 and the effect of COVID-19 on breast feeding and infant contact. Literature review was conducted to gather evidence of COVID-19 impact on perinatal outcome and provide a summary of most significant results. Four databases were used which includes EBSCOHost, PubMed, Scopus, and Google Scholar. Pregnant women with COVID-19 are at higher risk of severe outcomes compared to non-pregnant women with COVID19. In some studies, the incidence of pregnant women with COVID-19 who exhibit milder symptoms were higher than those with critical symptoms. Data on vertical transmission of COVID-19 from mother to child is equivocal. There was detectable SARS-CoV-2 RNA in breastmilk samples from mothers with COVID-19. Although Immunoglobin-A (IgA) and Immunoglobin-G (IgG) were present in their breastmilk samples, their immunity status against COVID-19 infection were unknown. The risk of acquiring serious symptoms of COVID-19 in infected pregnant women was higher than in infected non-pregnant women. It is still unclear whether COVID-19 can be vertically transmitted or whether breastfeeding is affected. Hence, further studies are needed to get better understanding of the COVID-19 implications on perinatal outcomes in aiming to reduce perinatal mortality and morbidity. 


\title{
POSTER PRESENTATION
}

\section{ID56 PP037}

\section{Digitalisation of birth registration and health systems in Malaysia: Boon or bane for the hard-to-reach and marginalised?}

\author{
Mohamed Razali, Rodziana'; Duraisingam, T.J.' ${ }^{2}$; T Mohd, TAM³. \\ ${ }^{1}$ Faculty of Syariah and Law, Universiti Sains Islam Malaysia, Nilai, Malaysia \\ ${ }^{2}$ Faculty of Business \& Law, Taylor's University, Subang Jaya, Malaysia \\ ${ }^{3}$ Faculty of Medicine and Health Sciences, Universiti Sains Islam Malaysia, Nilai, Malaysia
}

The 2030 Agenda for Sustainable Development adopts target 16.9 that ensures legal identity for all, measured by the percentage of children under five whose birth is registered. Legal identity that begins with birth registration increases access to basic rights and services including healthcare. In the early phase of the COVID-19 pandemic, the birth registration completeness in Malaysia drastically dropped from $100 \%$ in January 2020 to a mere $32.5 \%$ of the 11,369 births in March 2020 . The furthest left behind from birth registration and access to healthcare include the marginalised migrants, refugees and 'at-risk of stateless' populations. The objective of this paper is to comparatively examine digital and non-digital obstacles to birth registration and healthcare systems, including during the COVID-19 pandemic, among the hard-to-reach and marginalised migrants, refugees and at-risk of stateless populations in Peninsular Malaysia and Sabah. Data was gathered through desk review and online interviews using a mixture of close-ended and open-ended questions as part of a scoping exercise with a total of 16 participants among civil society organisations, community leaders and influencers representing the marginalised groups in this study. Data was comparatively analysed based on several themes constructed both inductively and deductively. Our results show unrecognised legal status and unaffordable costs of healthcare structurally hinder or limit access to birth registration and healthcare for both marginalised groups in Peninsular Malaysia and Sabah (ii) The marginalised populations in Sabah are further left behind compared to those in Peninsular Malaysia, mainly due to absence of smartphones and other digital devices, lack of literacy and technological knowledge and language barriers. COVID-19 amplifies the disadvantages based on the findings and calls for a blend of non-digital and digital development policies and strategies that first address the multidimensional determinants of barriers and divides among the furthest left behind. 


\title{
POSTER PRESENTATION
}

\section{ID57 PP038}

\section{Psychological Impact of COVID-19 Pandemic on Health Care Workers: A Scoping Review}

\author{
Shaari, Nurhafizah $^{1}$
}

${ }^{1}$ Faculty of Medicine and Health Sciences, Universiti Sains Islam Malaysia, Nilai, Negeri Sembilan, Malaysia

Frontline healthcare workers (HCWs) are among the most vulnerable populations at risk of mental health problems as the world battles the COVID-19 pandemic. Unchecked mental health problems can affect the HCW's work qualities and may jeopardize the health care services. The study aimed to identify the psychological impact of COVID -19 pandemic on the frontline HCWs. A scoping review using 'Arksey and O'Malley' framework was used to investigate the psychological impact on HCWs facing the COVID-19 pandemic. Four electronic databases (PubMed, ScienceDirect, ResearchGate and Google Scholars), dating between March to December 2020 were used to identify relevant articles. The search strategy included terms such as 'impact' OR 'effect' OR 'influence' AND 'healthcare workers' OR 'frontliner' AND 'psychology' OR 'mental health' OR 'emotional'. Only articles in English language were included. Initial search identified 1150 articles, and 5 articles of crosssectional studies were included after applying the inclusion and exclusion criteria. Findings suggest that the psychological impacts of COVID-19 on HCWs varies with several studies demonstrating an increased risk of acquiring stress, anxiety, depression, post-traumatic stress, and inadequate sleeping with different prevalence. Being a nurse, being female, and of younger age appeared to confer greater risk. Fear of the unknown, becoming infected or infecting others, poor family support, and overwhelmed with COVID-19 related news posed as mental health risk faced by the HCWs. This review demonstrates that the COVID-19 pandemic has a significant psychological impact on the frontline HCWs. The issues on mental health amongst the HCWs need to be addressed by the policymakers and relevant stakeholders, in which variety of supports must be offered to the HCWs who play a crucial role during the pandemic. This review also can guide current and future research in further prioritizing the maintenance of wellbeing amongst the frontline HCWs. 


\title{
POSTER PRESENTATION
}

\section{ID58 PP039}

\section{Correlation of Biomarkers Changes After Conventional Rehabilitation Therapy in Ischemic and Hemorrhagic Stroke Patients in Hospital Universiti Sains Malaysia}

$\underline{\text { Khiati Hadjer }^{1}}$; Hafiz, Muhammad Hanafi ${ }^{1}$; Muzaimi, Mustapha ${ }^{1}$; Kassim, Nur Karyatee ${ }^{2}$

\author{
${ }^{1}$ Department of Neurosciences, School of Medical Sciences, USM Health Campus, Kubang Kerian, Kelantan \\ ${ }^{2}$ Department of Chemical Pathology, School of Medical Sciences, USM Health Campus, Kubang Kerian, \\ Kelantan
}

Stroke is a heterogenous disease with multiple causes and risk factors. The pathophysiology of stroke is a complex process which leads to secretion of different blood makers into to blood circulation. This study evaluated the changes in serum concentration level of plasma CK, CTnT, IL-6 biomarkers and MBI scores after six weeks of the conventional rehabilitation therapy in ischemic and haemorrhagic stroke patients in USM. 19 participants (Males= 12, Females= 7, Age $M=52.79$; Ischemic $=9$, Hemorrhagic $=10$ ) received sessions of conventional physiotherapy for six weeks period. The sessions are based on conventional rehabilitation therapy done by similar sets of physiotherapists. Electrochemiluminescence immunoassay (ECLIA) which is a fully automated analyser was used to measure the concentration level of serum CK (The Architect c8000), Serum CTnT (Cobas e411) serum IL-6 (Cobas e601), and $\mathrm{MBI}$ was used to measure the functional outcome before the intervention and after the six weeks intervention. There are no statistically significant changes in, CK, CTnT, IL-6 and MBI scores before and after six weeks of conventional rehabilitation in ischemic and hemorrhagic stroke groups. Comparison between ischemic stroke group and hemorrhagic stroke group showed no significant differences $p>0.05(\alpha=0.05$ significant level), in all biomarkers' levels after 6 weeks conventional rehabilitation therapy. However, there was a significant difference in $\mathrm{MBI}$ scores ischemic and hemorrhagic stroke type $(\mathrm{p}=.006)$. The findings indicated that the changes in CK, and CTnT, IL- 6 are regulated by different mechanisms, and influenced by different factors which are related to the severity of the stroke, and a better functional recovery in stroke patients is related to the initial nervous system severity and dysfunction. Conventional rehabilitation therapy has positive effects on functional outcome in stroke patients. However, investigating the changes of the concentration level of blood biomarkers and MBI were limited due to the small sample size. 


\title{
POSTER PRESENTATION
}

\section{ID59 PP040}

\section{Online Teaching and Learning in Undergraduate Medical Education during COVID-19 pandemic: A Literature Review}

\author{
Ahmad Hamizi, Mohamed Aiman ${ }^{1}$; Ab Rahman, Siti Soraya ${ }^{1}$. \\ ${ }^{1}$ Faculty of Medicine and Health Sciences, Universiti Sains Islam Malaysia, Negeri Sembilan, Malaysia
}

There has been a sudden, rapid transition to online teaching and learning practices during the COVID-19 pandemic, involving almost all levels of education. Online methods of teaching and learning in higher education have been shown to be more flexible and able to cater to students with various levels of access. However, there is still a need for face-to-face delivery in teaching of practical, clinical and communication skills to undergraduate medical students. Various lockdown measures have removed students from the clinical environment, raising concerns on the effects on their training. This paper aims to review the impact of the pandemic on undergraduate medical education, specifically with regards to online teaching and learning. We also aim to identify the challenges faced in making this transition, and how medical students and teachers have adapted to these changes. Literature search using relevant keywords were performed on several databases such as PubMed and Google. During the COVID-19 pandemic, the Malaysian Ministry of Higher Education adopted online teaching and learning to replace face-to-face sessions. The undergraduate medical students were impacted by this move, as they missed out on vital workplace exposure from hospital attachment. Both students and teachers had to mutually adapt to online software like Google Meet and Zoom. Students found that they had more time to understand recorded lectures, but some missed the usual non-verbal communication experienced in physical encounters. Students also found that they had less opportunities to ask questions during online classes. A main drawback to online teaching is poor internet connectivity. Inability to cope with the rapid transition has also caused some students to suffer from anxiety and depression. Continuity of undergraduate medical programs is imperative even under lockdown measures. Medical students and teachers must adapt and overcome challenges to ensure quality of training is achieved. 


\section{POSTER PRESENTATION}

\section{ID61 PP041}

\section{The Potentials of Smartwatches for Self-Health Monitoring}

Rafee, Faris' ${ }^{1}$; Faizal, Auni' ${ }^{1}$; Azlan, Azzam'; Nasir, Maasyah'1 ; Azmi, Liyana'; Norazmi, Abu 'Ubaidah Amir'

${ }^{1}$ Faculty of Medicine \& Health Sciences, Universiti Sains Islam Malaysia, Nilai, Negeri Sembilan, Malaysia

Wearable Health Devices (WHDs) is a form of technology that enables continuous ambulatory monitoring of human vital signs daily or in a clinical setting. Smartwatches represent the most popular type of wearable devices and enables selfmonitoring of personal activity through the measurement of heart rate variability, blood pressure, steps taken and daily walking distance. Therefore, the application of smartwatches can and should be applied to improve our well-being. We aim to review the uses of smartwatches in improving wellbeing in daily and clinical settings. We used search strategy using some of the keywords such as "wearable devices", "smartwatch" and "healthcare". Literature review was done using Google Scholar, Medline and PUBMED electronic databases, journals, and articles on relevant and associated topics. Studies have showed that smartwatches are vital tools for chronic disease self-management interventions such as Parkinson's disease, epilepsy, diabetes, asthma, and visual impairment. Reeder (2016) emphasized the usage of smartwatches on activity monitoring, heart rate monitoring, speech therapy adherence, diabetes self- management, and detection of seizures, tremors, scratching, eating and medication-taking behaviors. Smartwatches can be utilized to monitor patients in nursing facilities and their homes for specific health events to improve quality of life. The most recent study also shows the ability of smartwatch to monitor for arrhythmias and QT interval prolongation in COVID-19 patient during home isolation. Notably, limitations of smartwatch use in both daily and clinical settings such as its high cost, lack of app compatibility and database heterogeneity must be considered. Finally, data accuracy and confidentiality were also considered main concerns for smartwatch users. Smartwatches have vast potential in monitoring and improving patient care for chronic diseases. However, significant research on much larger populations is necessary to determine their acceptability and effectiveness in clinical practice. 


\section{POSTER PRESENTATION}

ID63 PP042

\section{The Magic of MySejahtera - Contact Tracing and COVID-19 Vaccination Management in Malaysia}

Faizal, Auni ${ }^{1}$; Nasir, Maasyah ${ }^{1}$; Azlan, Azzam ${ }^{1}$; Rafee, Faris $^{1}$; Azmi, Liyana ${ }^{1}$

${ }^{1}$ Faculty of Medicine \& Health Sciences, Islamic Science University of Malaysia, Nilai, Negeri Sembilan, Malaysia

MySejahtera is a mobile application developed by the National Security Council (NSC), the Ministry of Health (MOH), and Malaysian Communications and Multimedia Commission (MCMC). It is used to facilitate the process of contact tracing and manage the COVID-19 vaccination appointments in Malaysia. Other features of MySejahtera include risk assessment, teleconsultation, and COVID-19 hotspot tracking. Approximately $70 \%$ of the population of Malaysia has downloaded MySejahtera with recorded use of 17 million check-ins per day. We aim to review effectiveness of MySejahtera usage in contact tracing and vaccination management during COVID-19 outbreak. We used search strategy using some of the keywords such as "MySejahtera", "contact tracing", "vaccination" and "COVID-19". Literature review was done using Google Scholar, PUBMED, web sources, journals, and articles on relevant and associated topics. Epidemiological studies utilise the database from MySejahtera to perform contact tracing, to anticipate infection clusters and manage COVID-19 infections. Statistics showed that $15.1-37.8 \%$ cases detected through contact tracing data will be gathered using the MySejahtera Check-In module. Thus, Mysejahtera can be used to curb COVID-19 incidence and inhibit resurgence of infections in future global health crises. Vaccination registration in Malaysia is also managed via MySejahtera. According to Special Committee on COVID-19 Vaccine Supply (JKJAV), 26.56\% of Malaysians aged 18 and above have registered for COVID19 vaccine via MySejahtera as from March 2020 and rising to $72.7 \%$ as for 13 July 2021. However, privacy, practicability, security and smartphone-limitations could be the significant limitations concerning of MySejahtera usage amongst civilians. MySejahtera shows promise in assisting COVID-19 contact tracing and vaccine management in managing the COVID-19 outbreak in Malaysia. Future studies could address the limitations of MySejahtera and use the wide user-database for other digital health applications. 


\section{POSTER PRESENTATION}

\section{ID068 PP044}

\section{Clinical Engagement Through Telemedicine During COVID-19 Pandemic \& Going Beyond}

Muhammad, Amalin Kamilah ${ }^{1}$; Mohamad Nazarallah, Mohamad Hilmi ${ }^{1}$.

${ }^{1}$ Faculty of Medicine and Health Sciences, Universiti Sains Islam Malaysia

The world recently has been alarmed with a revolutionizing situation involving the COVID-19. The increment of cases recorded each day brings about concerns that it will put everyone, especially the healthcare workers at high risk of contracting the virus. This in turn has led to a paradigm shift whereby most physical activities are shifting towards virtual platforms including a good proportion of health systems through telemedicine or telehealth. The COVID-19 pandemic has hastened the adoption of telemedicine and it is now widely utilized and may lead to a new norm. The aim of the study is to assess the feasibility of telemedicine and to deduce the overall response of adoption of technology in Malaysia healthcare system through the scientific publications that encompass telemedicine prior and during COVID-19 pandemic. Electronic search was performed through Medline Complete, Academic Search Complete and Pubmed where the following MeSH terms were used; telemedicine or telehealth, COVID-19 or 2019-NCoV or SARS-CoV-2, and Malaysia. All studies demonstrated positive acceptance and outcomes despite few challenges and drawbacks of telemedicine usage in teleconsultation, teleradiology, teleconference, telemonitoring and other virtual meetings. Use of telemedicine eliminates the location barrier in exchange of information to procure diagnosis, treatment \& prevention of disease as well as continuous medical education among health care personnel to facilitate individuals' as well as their communities' health. Telemedicine in Malaysia plays a key role in the health care system especially in the light of the COVID-19 pandemic though its potential benefits have not been entirely explored due to lack of validated studies and reports regarding the subject matter. It may not be able to replace physical health care functions, but it does have sufficient ability to support a particular portion of the health care delivery system for greater efficiencies. 


\title{
POSTER PRESENTATION
}

\section{ID070 PP046}

\section{Sudden Sensorineural Hearing Loss in COVID-19: Our Experience}

\author{
Lye Meng Hon ${ }^{1,2}$, Carren Teh Sui Lin², Nik Adilah Binti Nik Othman ${ }^{1}$ \\ ${ }^{1}$ Department of Otorhinolaryngology-Head and Neck Surgery, School of Medical Sciences, Universiti Sains \\ Malaysia, Health Campus, Kubang Kerian 16150 Kelantan, Malaysia. \\ 2 Department of Otorhinolaryngology-Head and Neck Surgery, Hospital Sungai Buloh, Jalan Hospital, 47000 \\ Sungai Buloh, Selangor, Malaysia
}

Coronavirus disease-2019 (COVID-19) is responsible for unparalleled mortality and morbidity worldwide. The commonest otolaryngologic symptoms reported were cough, anosmia, and sore throat. Sudden onset sensorineural hearing loss (SSNHL) among COVID-19 patients was reported sparingly. Here we present a case of SSNHL in COVID-19 and the challenges we faced. A 55-year-old lady with underlying renal failure was admitted for category 3 COVID-19. During admission, she experienced sudden deafness in both ears. No other neurological deficit was detected. Otoscopic findings were unremarkable. Screening for connective tissue diseases were negative. Bedside auditory brainstem response (ABR) test was done in the isolation ward by clinicians in full protective equipment revealed left profound and right severe hearing loss. Portable ABR equipment was wrapped in clear plastic film and sanitized with $70 \%$ isopropyl alcohol then discarded after use. Favipiravir which started on admission was withheld. She was given oral prednisolone and tapered off over 2 weeks. Imaging was not done due to restriction on COVID-19. Patient reported improvement in hearing. However, she was only seen in outpatient clinic after 3 months. Pure tone audiometry showed right moderate to severe and left severe to profound sensorineural hearing loss with normal tympanometry. Patient was advised for hearing aids. Multiple mechanisms were proposed as cause of SSNHL in COVID19. Favipiravir is approved for use in mild to moderate COVID-19 infection. Hearing loss is not an established adverse drug event. Presently, there is no guideline for hearing disorder in COVID-19. SSNHL is an acute otologic disorder and should be treated immediately with oral or intertympanic steroid and close monitoring. Patient presenting with sudden onset hearing loss should be screened for COVID-19. Audiologic tests and treatment with steroid should be started promptly. Much research is required to fully understand the impact of COVID-19 on the auditory system. 


\title{
POSTER PRESENTATION
}

\section{ID73 PP047}

\section{Contributing Factors of LMA Usage in Pre-Hospital Care Among Assistant Medical Officers}

\author{
Abdul Ghafar, Muhammad Ariffin ${ }^{1,2}$; Naganathan, Ratneswaran ${ }^{2,3} ;$ Mamat, Wahidi ${ }^{2,3}$; Zainol, Zohori ${ }^{2,4}$. \\ ${ }^{1}$ Department of Emergency and Trauma, Hospital Shah Alam, Selangor, Malaysia. \\ ${ }^{2}$ Board of Medical Assistant, Medical Practice Division, Ministry of Health Malaysia, Putrajaya, Malaysia. \\ ${ }^{3}$ Hospital Supervisor Unit, Hospital Tengku Ampuan Rahimah Klang, Selangor, Malaysia. \\ ${ }^{4}$ Hospital Supervisor Unit, Hospital Shah Alam, Selangor, Malaysia. \\ ${ }^{5}$ Department of Orthopaedics, Hospital Shah Alam, Selangor, Malaysia.
}

Laryngeal Mask Airway (LMA) learning and practice were compulsory before being registered as an Assistant Medical Officer (AMO) in the Board of Medical Assistant. The practice has been comparatively slow and currently, there is no study locally known among AMO to look at the usage of LMA in pre-hospital care (PHC). The purpose of this study is to estimate the prevalence of LMA usage among AMO's to perform insertion in PHC settings and identify the contributing factors which encourage them in their practice. This cross-sectional study used questionnaire distributed in 2018 among Hospital Sungai Buloh's paramedics and eight nearby clinics. Retrospective data of PHC reports between 2016-2017 was reviewed for total LMA practice. Descriptive and analytical statistics were performed. 43 samples involved, whereby majority are male in $25-29$ of age group. $81.4 \%$ are encouraged to use LMA and 69.8\% perform a total of 38 insertions in PHC. 35 respondents were exposed to a Compulsory Placement Program (PPW). 27.5\% LMA used in Out of Hospital Cardiac Arrest (OHCA) cases in hospital's registry. Cross-tabulation analysis shows support from supervisors and organizations (96.7\%) and attending airway courses $(80.0 \%)$ were the key factors besides PPW, age factors, credentialing and others training. Moral support and courses are the most contributing factors among AMO's in PHC. The factors may vary through working situations and supportive management groups to maximize the usage of LMA among AMO hence increases chances of patients' survival rates. 


\title{
POSTER PRESENTATION
}

\section{ID74 PP048}

\section{Effectiveness of the Teleaudiology Approach for Hearing Assessment and Intervention: A Comprehensive Review}

Rashid, Mohd Fadzil Nor'; Hussain, Mohd Khary²; Mahmud, Halimah²; Ahmad, Rozazipah; Mansor, Suzana; Mohd Zainudin, Nur Syazana².

\author{
${ }^{1}$ Audiology Programme, School of Health Sciences, Health Campus, Universiti Sains Malaysia, Kubang Kerian, \\ Kelantan, Malaysia \\ ${ }^{2}$ Audiology and Speech Pathology Unit, Hospital Universiti Sains Malaysia, Health Campus, Universiti Sains \\ Malaysia, Kubang Kerian, Kelantan, Malaysia
}

Teleaudiology refers to the technology used to deliver audiology and information services to clients using information communication technology applications. Several systematic reviews have addressed the hearing assessment and intervention using teleaudiology approach, but there is still uncertainty about their effectiveness in diagnosing hearing status and intervention programs for deaf and hard of hearing patients. This comprehensive review aimed to i) measure and compare the overall validation and reliability of teleaudiology testing, ii) determine the effectiveness of hearing interventions for patients, and iii) better understand how audiologists perceive or experience while using teleaudiology approach. An iterative search strategy for six bibliometric databases and grey literature was carried out to identify English-language publications. All systematic reviews included were presented with Preferred Reporting Items for Systematic Reviews and Meta-Analyzes (PRISMA) and were critically assessed using standard tools from the Joanna Briggs Institute (JBI). The data were extracted with a JBI data extraction tool independently by the first author and verified by all authors during the online discussion. A narrative synthesis approach was applied to the result. This comprehensive review included six systematic reviews, with 12 relevant randomized controlled trials and 2709 participants. Overall, four systematic reviews involved adults, and one systematic review focused on pediatric populations. For the validity and reliability of the teleaudiology testing, only one systematic review had been discussed in general. Five systematic reviews had examined the effectiveness of intervention and highlighted the outcome measures, hearing aid rehabilitation procedures and speech-language development outcomes. Meanwhile, only one systematic review focused on details regarding the experience using teleaudiology among audiologists. From the included studies, most of the systematic reviews focused on teleaudiology approaches for interventions. Future evidence should be sought and synthesized in the domains of hearing assessment and experiences using teleaudiology approach. 


\section{POSTER PRESENTATION}

\section{ID75 PP049}

\section{Physical and Mental Health Impacts of Watching Mukbang in Young Adult}

Zulkefli, Siti Hajjar ${ }^{1}$; T Mohd, Tengku Amatullah Madeehah ${ }^{1}$.

${ }^{1}$ Faculty of Medicine and Health Science, Universiti Sains Islam Malaysia, Nilai, Malaysia

Mukbang is a term derived from a combination of two Korean words which are 'meokneun' and 'bangsong'. 'Meokneun' means eating while 'bangsong' means broadcast, thus translating it to "eating broadcast" in English. It is an eating show where people record a video of themselves eating while leisurely chatting with the viewers. A person who creates the content of mukbang is called a mukbanger. Today, mukbang can be found everywhere in various forms of social media. This study was conducted to explore the contents of mukbang and its physical and mental health impacts on young adults. A scoping review was carried out using the following electronic databases: PUBMED, Google Scholar, UpToDate and Scopus. The keywords used were "young adult", "student", "teenager", "mukbang", "eating show", "eating video", "online eating broadcast", "collaborative eating", "physical health" and "mental health". From the search, 492 journals and articles were screened. The inclusion criteria were primary studies conducted among the adult population and published in the English language. A total of 7 articles were included in this study. It was identified that most mukbangers challenged themselves to eat an extremely large amount of spicy food and highlighted their eating sound. Watching mukbang is associated with negative effects such as obesity, alteration of food preferences, modelling of bad eating behaviours, promoting disordered eating, and internet addiction. Meanwhile, watching mukbang can also be used as a constructive tool to reduce feelings of loneliness and social isolation, build a virtual social community and provide vicarious satisfaction for the viewers. Mukbang can be both helpful and destructive at the same time depending on the viewer's context. Further longitudinal or cohort studies should be initiated to investigate the long term outcomes of mukbang and develop strategies for the prevention of physical and mental health problems associated with mukbang. 


\title{
POSTER PRESENTATION
}

\section{ID76 PP050}

\section{Hand Grip Strength and its Associated Factors Among E-sports And Video Gaming Players: A Cross-sectional Study Protocol}

\author{
Mohd Kamil, Muhammad Kashani'; Muhammad Yazid, Muhammad Ashraff ${ }^{1}$, Ismail, Khairil Idham²; Salim, \\ Ahmad Munawwar Helmi $;$; Amir Norazmi, Abu Ubaidah. \\ ${ }^{1}$ Sports Medicine Unit, University of Malaya (UM). \\ ${ }^{2}$ Department of Community Health, Faculty of Medicine, Universiti Kebangsaan Malaysia Medical Centre. \\ ${ }^{3}$ National Sports Institute of Malaysia (NSI). \\ ${ }^{4}$ Faculty of Medicine and Health Sciences, Universiti Sains Islam Malaysia.
}

E-sports and video gaming involve low impact but highly repetitive activities commonly in wrist and hands. This could potentially lead to a weak hand grip strength (HGS) and a significant musculoskeletal injury. However, little is known about the relationship between HGS, musculoskeletal injuries of the wrist and hands and its associated factors among E-sports and video gaming practitioners. We aim to investigate the relationship between HGS, musculoskeletal injuries of wrist and hands, and other associated factors among $\mathrm{E}$-sports and video gaming practitioners. This is a cross-sectional study protocol involving $111 \mathrm{E}$-sports and video game players who fulfilled the inclusion criteria. Sociodemographic, anthropometric measurements, bioimpedance analysis, level of competitions, hours spent in training, and types of gaming consoles usage will be obtained. HGS will be measured by using Jamar dynamometer along with clinical assessment of wrist and hands by sports physician. Linear regression analysis will be used to assess the association between HGS, musculoskeletal injuries and other risk factors. We hypothesize that HGS are associated with musculoskeletal injuries of wrist and hands such as tendinopathic or neuropathic conditions, including wrist and hands flexor and extensor tendinopathies, de Quervain's tenosynovitis, gamer's thumb, intersection syndrome, and carpal tunnel syndrome. Other associated factors can include age, body mass index (BMI), level of competitions, training hours, and types of gaming consoles. In conclusion, weak hand grip among E-sports and video gaming practitioners could increase susceptibility to wrist and hands musculoskeletal injuries other than lifestyle habits, ergonomics, biomechanics, and training hour factors. This finding would benefit physicians, physical therapists, or athletic trainers to start injury prevention programmes, return-to-play protocol, as well as treatment interventions. As a result, E-sports and video gaming practitioners can receive adequate therapy for musculoskeletal problems of the wrist and hands. 


\title{
POSTER PRESENTATION
}

\section{ID77 PP051}

\section{LGBT: An Uprising Worldwide Phenomenon - A Systematic Review on Academics and Psychological Impacts among Children with LGBT Parents}

\author{
Ahmad Affandi, Arni Farisha1; Mohamad Nor, Nadeeya 'Ayn Umaisara² \\ ${ }^{1}$ Faculty of Medicine and Health Science, Universiti Sains Islam, Nilai, Negeri Sembilan, Malaysia \\ ${ }^{2}$ Community Health Unit, Faculty of Medicine and Health Sciences, Universiti Sains Islam Malaysia
}

Several health implications have been associated with Lesbian, Gay, Bisexual and Transgender (LGBT) community. However, the number of LGBT individuals keep increasing together with the number of children with LGBT parents. To systematically identify and review evidence between 2010 to 2021 regarding the academics and psychological impacts of children with LGBT parents. The study methodology is following Preferred Reporting Items for Systematic Reviews and Meta-Analysis (PRISMA) guidelines, the search was conducted in PubMed, ProQuest, Springer Link, Google Scholar, Science Direct, Research Gate and EBSCOHost using various keywords such as "children", "child", "homosexual/transgender/ bisexual parents"," academic", "psychological”, "depression”, "anxiety”, "stress”, "mental health", "impact", "effect”, "LGBT parents", "same-sex couples". Inclusion criteria was identified using PICOS, namely Population: children with LGBT parents, Comparison: LGBT parents, Output: academic, psychological, Study design: crosssectional and longitudinal study. Ten articles fulfilled all the inclusion criteria and were critically reviewed. Results showed that academic impacts were varied due to different academic assessment tools used in the three studies while no psychological impact was reported among children with LGBT parents. Inconclusive findings with limitations derived from this systematic review require further investigation. Future unbiased research is necessary to explore the impact of having LGBT parents towards children's academic and psychological well-being as stigmatization and criminalization are reported in this community. 


\section{POSTER PRESENTATION}

\section{ID78 PP052}

\section{The Impact of COVID-19 to Healthcare Service Utilization in Malaysia: A Scoping Review}

Izani, Izlyn'; Jaafar, Hafiz'; Mohd Nor M³.

${ }^{1}$ Faculty of Medicine and Health Sciences, Universiti Sains Islam Malaysia, Bandar Baru Nilai, 71800, Nilai, Negeri Sembilan, Malaysia

${ }^{2}$ Public Health Unit, Department of Primary Health Care, Faculty of Medicine and Health Sciences, Universiti Sains Islam Malaysia, Bandar Baru Nilai, 71800, Nilai, Negeri Sembilan, Malaysia

${ }^{3}$ General Surgery Unit, Faculty of Medicine and Health Sciences, Universiti Sains Islam Malaysia, Bandar Baru Nilai, 71800, Nilai, Negeri Sembilan, Malaysia

COVID-19 pandemic has put a significant strain on the healthcare system globally. Major adjustments in the healthcare system and reallocation of resources, supplies and manpower were done to cater the overwhelming demand of COVID-19 patients. The aim of this study is to collect, analyze and summarize articles that study changes in healthcare services utilization including changes in clinic visit, ward admission, diagnostic and therapeutic procedures during the COVID-19 pandemic in Malaysia. A scoping review of literatures that were systematically searched by using four electronic databases (EBSCOhost, Scopus, PubMed and Google Scholar). All published articles between year 2020 to year 2021, that measures the utilization of healthcare services during COVID-19 pandemic and compared with a corresponding period before the pandemic were included in this study. Case reports and studies reporting on healthcare services utilization by COVID-19 patients were excluded from the study. Six studies were included in the final analysis. The main methodology used were observational analytic and observational descriptive. Data were mainly drawn from international information systems and registries, medical records from single health facility and online surveys. All of the studies demonstrated the reduction in number of surgeries performed ranged from $23 \%$ to $83 \%$, reduction in outpatient clinic volume with mean of $57.8 \%$ and reduction in ward admission of dengue cases ranged from $35.85 \%$ to $57.69 \%$. Telemedicine has become an alternative method in patient management and care. Healthcare services, delivery and utilization of non COVID-19 cases in Malaysia had decreased during the COVID-19 pandemic. The main factor is mainly due to administrative instruction as an effort to reduce the risk of COVID-19 infection. 


\title{
POSTER PRESENTATION
}

\section{ID83 PP055}

\section{A Case of Salivary Gland Choristoma Presenting with Ear Discharge in a Child with Canal Stenosis}

\author{
Mohd Saiful Al ${ }^{1,2}$, Nik Adilah NO ${ }^{1,2}$, Farhana MM2,3, Sharifah Emilia.TS ${ }^{2,3}$, Mohd Khairi MD ${ }^{1,2}$. \\ ${ }^{1}$ Department of Otorhinolaryngology-Head \& Neck Surgery, School of Medical Sciences, Universiti Sains Malaysia \\ Health Campus, 16150 Kubang Kerian, Kelantan, Malaysia. \\ ${ }^{2}$ Hospital Univerisiti Sains Malaysia, 16150 Kubang Kerian, Kelantan, Malaysia. \\ ${ }^{3}$ Department of Pathology, School of Medical Sciences, Universiti Sains Malaysia Health Campus, 16150 Kubang \\ Kerian, Kelantan, Malaysia.
}

A unilateral conductive hearing deficit is typically the first manifestation of choristoma, with otorrhoea and occasionally a mass in the middle ear. This lesion may be associated with deformed or absent ossicles as well as branchial arch and facial nerve abnormalities. A two month old baby girl was under the follow up of the ORL clinic. She was born with right microtia and canal stenosis. She also had right facial nerve palsy, as evidence by slight deviation of the right angle of the mouth and loss of nasolabial fold. The mother noticed that there was on and off mucoid discharge from the right ear which improved after topical ear drops. However, in the last eight months old, she noticed an intermittent fluctuant swelling measuring $2 \times 2 \mathrm{~cm}$ with erythematous skin from the right infra-auricular area. Initially, it was treated with oral antibiotics. However, the swelling did not resolve after the third episode hence aspiration was performed which yielded 3cc of pus. Upon follow up at 1-year-old, the aspirated area was fibrosed with formation of cutaneous fistula containing occasional mucoid discharge. The extent of the excision of salivary gland choriostoma is controversial. Some authors thought that salivary heterotopia consists of normal tissue and, thus, did not require complete excision when diagnosed histologically. However, when there are signs of infection or neoplasm, it requires complete removal of the lesion. The possibility of malignant transformation is very rare, but theoretically, choristomas are immature, and this itself increases the chance of malignancy. Total excision is recommended to avoid recurrent infection in the future. In this patient, the lesion is fully excised due to recurrent infection to her right ear secondary to the fistula in the ear canal. 


\section{POSTER PRESENTATION}

\section{ID85 PP057}

\section{COVID-19 Pandemic: Increasing in Gadget and Internet Addiction Among Children}

Rahim, Huda ${ }^{1}$; Mokhtar, Azlina ${ }^{1}$

${ }^{1}$ Faculty of Medicine and Health Sciences, Universiti Sains Islam Malaysia (USIM), Nilai, Negeri Sembilan, Malaysia

The COVID-19 pandemic has caused school closures, quarantines, and the rise of online learning affecting millions of children. Childhood gadget and internet addiction has skyrocketed since the outbreak. The aim of this study is to look at the relationship between gadget and internet addiction with COVID-19 pandemic among children including its prevalence, contribution factors and impact toward their health. A literature review on available studies from online databases including PubMed, Scopus, ScienceDirect, and Google Scholar that directly linked to the topic were reviewed and analyzed accordingly. The searched keywords were children, COVID-19, Internet addiction, factors, and impact. The prevalence of gadget and internet addiction among children during pandemic increased in all studies that were reviewed. In Malaysia 70.5\% were assessing the internet from home after movement control order (MCO) implementation. Social networking activities rose from $85.6 \%$ in 2018 to $93.3 \%$ in the year 2020. Other online activities such as downloading online videos and playing online games also showed a tremendous increase. In the United States $48.0 \%$ of children spent more than six hours per day on the screen and it increased up to $500 \%$ compared to the previous years. Increased frequency and duration of internet usage during pandemic leads to $33.37 \%$ of children in China fall into problematic internet users, and $2.68 \%$ to categorized as addictive internet disorder. There were several contributing factors including permissive parenting style, children's preference, and peer pressure. Significant impact can be seen through their mental health such as depression and anxiety as well as physical health concerns for example myopia, obesity, and insomnia. COVID-19 pandemic and increased gadget and internet addiction among children are related. International health organisations, child welfare organisations, governments, and the children's families themselves must work together to minimise the devastating consequences of COVID-19 on children. 


\section{POSTER PRESENTATION}

\section{ID86 PP058}

\section{Susceptibility to COVID-19 and Illness Severity in Elderly Patients: A Comparison with Paediatric Age Group}

Romizhi, Aliah Fatin ${ }^{1}$; Nor Aripin, Khairun Nain².

Faculty of Medicine and Health Science, Universiti Sains Islam Malaysia, Nilai, Malaysia

On March 11, 2020, the World Health Organization (WHO) declared the Coronavirus disease (COVID-19), caused by the severe acute respiratory syndrome coronavirus 2 (SARS-CoV-2) a pandemic. Age-related predisposition and the illness severity of the disease are being analyzed extensively. Illness severity is one of the most important issues to address as it may give impact to the triage, management, and prognosis of every patient. Severe, often fatal, respiratory problems are said to develop more frequently in elderly individuals due to their age-related impaired immune response. This study was conducted to explore and associate the epidemiological characteristics and illness severity of COVID-19. Related articles were reviewed and analyzed from the search engine using the following electronic databases: Google Scholar, PUBMED and UpToDate. The most searched keywords were "COVID-19", "elderly", "paediatrics", "age-related", "illness severity" and "predisposition". The case fatality rate and risk of developing severe COVID-19 are significantly higher in elderly. Advanced age itself contributes significantly to developing symptomatic to severe COVID-19 that leads to a higher mortality rate. Meanwhile children at all ages appeared far less susceptible to COVID-19 symptoms once they are exposed to the virus. A possible reason is that children have certain physiological characteristics that prevent the harmful effects caused by the SARS-CoV-2 virus. Children have fewer Angiotensin Converting Enzyme 2 (ACE2) receptors, the entry point for the virus. However, children can also be seriously affected, such as the reported Multisystem Inflammatory Syndrome (MIS). Worryingly, strained health systems can have a devastating effect on child health especially in less developed countries. The paediatric age group appears to be far less affected by COVID-19. Further studies are needed to elucidate this age-related difference in susceptibility. 


\title{
POSTER PRESENTATION
}

\section{ID87 PP059}

\section{Neglected Foreign Body Oesophagus Complicated by Tracheo- Oesophageal Fistula}

\author{
Mohamad Najib bin Salleh ${ }^{1,2}$, Azliana Aziz ${ }^{1}$, Hashimah Ismail ${ }^{3}$, Wan Zainira Wan Zain ${ }^{4}$, Mohd Nizam Md Hashim \\ Irfan Mohamad 1

\begin{abstract}
${ }^{1}$ Department of Otorhinolaryngology-Head and Neck Surgery, School of Medical Sciences, Universiti Sains Malaysia Health Campus, 16150 Kota Bharu, Kelantan, Malaysia.

Department of Otorhinolaryngology-Head and Neck Surgery, Hospital Tuanku Fauziah, Kangar, Perlis, Malaysia. ${ }^{3}$ Department of Otorhinolaryngology-Head and Neck Surgery, Hospital Raja Perempuan Zainab 2, Kelantan, Malaysia

${ }^{4}$ Department of Surgery, School of Medical Sciences, Universiti Sains Malaysia Health Campus, 16150 Kota Bharu,
\end{abstract} \\ Kelantan, Malaysia.
}

Accidental foreign body (FB) ingestion is common in patients of advanced age. Approximately $10 \%-20 \%$ of cases of foreign body ingestion necessitate endoscopic removal, while less than $1 \%$ of surgery was required to remove the FB or treat the complications. Denture prosthesis ingestion in an adult show about $3.1 \%$ of the cases. Due to the entrapment of denture wire hooks in the oesophageal wall, trial removal of the dental prosthesis during esophagoscopy can result in oesophageal perforation. Oesophageal perforation is a serious condition requiring immediate attention. It has a high mortality rate of $5 \%$ to $40 \%$ and can be made worse by delayed diagnosis and treatment, leading to trachea-oesophageal fistula. CT scan with $100 \%$ sensitivity, 92.6\% specificity, $97.9 \%$ positive predictive value, and 100\% negative predictive value to detect $F B$ in the oesophagus. It also helps us identify the site of oesophageal perforation and complications arising from the embedded FB. The closure of an oesophageal perforation can be accomplished in various ways, including stenting, and clipping. Still, in general, small oesophageal perforation can be treated conservatively. As in this case, we managed to remove the whole FB during esophagoscopy and subsequently noted that the patient had a small tracheoesophageal fistula managed by surgical clipping. Postoperatively patient well tolerated orally with no more tracheal-oesophageal fistula seen by barium swallow confirmation. Proper, thorough history taking, and clinical examination are mandatory in case of FB at the throat. Never underestimate the patient's presenting complaint as it may lead to improper diagnosis and patient management. 


\title{
POSTER PRESENTATION
}

\section{ID91 PP061}

\section{A Systematic Review: Consideration of The Psychological Impact on Male Partners in The Management of Early Pregnancy Loss}

\author{
Karali Hassan Fadhil ${ }^{1}$; Poh, Dawn Siaw Celine ${ }^{1}$; Phong, Yen Wen ${ }^{1}$; Soorianarayanan, Parveena ${ }^{1}$; Ting Jason \\ Siong ${ }^{1}$; Dr Zaigham Muhammad Talha ${ }^{2}$
}

\author{
${ }^{1}$ Newcastle University Medicine Malaysia (NUMed), Johor, Malaysia \\ ${ }^{2}$ Universiti Malaysia Sabah (UMS), Kota Kinabalu, Malaysia
}

Pregnancy is still arguably considered primarily a "women's issue". Most international and local guidelines focus on the psychological impact of early pregnancy loss on mothers with, unfortunately, little to no acknowledgement of the male partner. The study aimed to highlight the psychological effects of various types of early pregnancy loss on the male partner and explore national and international guidelines' consideration of male partners' management. The OVID MEDLINE $®$ without Revisions database was searched from the year 1946 until August Week 2 2020 (Current). The Embase database was searched from the year 1974 until Week 342020 (Current). 2458 papers (1236 from OVID MEDLINE $®$ without Revisions; 1222 from Embase) were retrieved. 42 papers were accepted. Due to inadequate coverage from both databases on molar and ectopic pregnancies, an additional 6 from Google Scholar were added, making the total 48 articles for review. Articles were summarized and reviewed qualitatively to extract themes revolving the psychological impact of early pregnancy loss on the male partner. All available guidelines were reviewed. Themes were compared to obtain results for discussion. Feelings of the male partner were diverse in different types of early pregnancy loss, such as feelings of grief, sadness, depression, anguish, and devastation. However, relief after the incident was experienced in some conditions. Male partners were expected to be strong despite their own negative feelings, and they complained of lack of recognition of grief and support. Revision of international guidelines for early pregnancy loss management show no clear protocols in the involvement, counselling, or support of male partners during or after the process. Male partners experience a range of emotional responses towards early pregnancy loss which often go overlooked. Hence, they should be actively included in clinical consultations involving early pregnancy loss and be supported by healthcare facilities throughout the process. 


\section{POSTER PRESENTATION}

\section{ID93 PP063}

\section{The Association of Dietary Intake and Acne Vulgaris: A Naturopathic Perspective}

Wan Anuar, Wan Nur Adlina'; Mohamad Nor, Nadeeya 'Ayn Umaisara'; Abu Hasan, Zatul- 'Iffah'1

${ }^{1}$ Faculty of Medicine and Health Sciences, Universiti Sains Islam Malaysia, Nilai, Malaysia.

Acne or medically named acne vulgaris is a chronic skin disorder that affects over $90 \%$ of males and $80 \%$ of females in the population aged 11 to 30 -year-old. Even though acne is perceived as a trivial, self-limiting and cosmetic disorder; it is not a petty problem that can be ignored as social isolation, depression and suicidal ideation are frequent comorbidities observed with this disease. Various forms of acne therapies exist and have their own benefits, but they also come with unintended side effects. Hence, comprehensive knowledge on the type of diet that can influence exacerbation of acne are essential as an alternative for acne intervention and prevention. This study was conducted to review and determine the association between several dietary variables (dairy product, glycemic load, chocolate and fatty acids) and acne vulgaris. PUBMED-MEDLINE database and Google Scholar were used for a thorough literature search for articles published over 5 years (2017-2021) using the following keywords: "acne vulgaris", "dietary influences", "glycemic index", "dairy product" and "fatty acid". This study presents the current evidence on the potential impact of diet on the pathogenesis of acne. Convincing evidence shows that dairy products and high glycemic index diets are conclusively associated with acne vulgaris phenomenon as they lead to an increase in sebum production which plays a vital role in acne development. However, the relationship between chocolate and acne remains unclear and inconclusive as the vagueness may be associated with the chocolate type, ingredients and preservatives. Meanwhile, omega- 3 fatty acids had been proved to have a protective role against acne development. Diet-acne hypotheses were predominantly based on observation and speculation. Even though the influence of diet on acne vulgaris remains controversial, it cannot be overlooked as it may be one of the preventive measures that can be advised to the patients apart from the other acne therapies. 


\title{
POSTER PRESENTATION
}

\section{ID95 PP065}

\section{Giant Botryoid Fibroepithelial Polyp as an Unusual Cause of Obstructive Uropathy in a 6-Year-Old Girl}

\author{
Azzahra Azhar 1,6,7, Mohamad Ikram llias ${ }^{2,6}$, Mohamed Ashraf Mohamed Daud ${ }^{4,6}$, Ahmad Hadif Zaidin \\ Samsudin ${ }^{3,6}$, Nur Asyilla Che Jalil,1,6 \\ ${ }^{1}$ Department of Pathology School of Medical Sciences, Universiti Sains Malaysia, Health Campus, Kubang \\ Kerian, Kelantan, Malaysia. \\ ${ }^{2}$ Departmen of Paediatric Surgery, School of Medical Sciences, Universiti Sains Malaysia, Health Campus, \\ Kubang Kerian, Kelantan, Malaysia. \\ ${ }^{3}$ Department of Radiology, School of Medical Sciences, Universiti Sains Malaysia, Health Campus, Kubang \\ Kerian, Kelantan, Malaysia. \\ ${ }^{4}$ Department of surgery, School of Medical Sciences, Universiti Sains Malaysia, Health Campus, Kubang \\ Kerian, Kelantan, Malaysia. \\ ${ }^{6}$ Hospital Universiti Sains Malaysia, Kubang Kerian, Kelantan, Malaysia. \\ ${ }^{7}$ Faculty of Medicine, Universiti Sains Malaysia, Kuala Terengganu, Malaysia.
}

Fibroepithelial polyps (FEP) of the urinary bladder is a rare benign tumour in the paediatric age group which arises from mesoderm. We reported a case of a six-yearold girl who presented with persistent haematuria and obstructive uropathy. She is on regular haemodialysis due to her high urea and creatinine level. Computed tomography revealed a large heterogenous polypoidal mass in the urinary bladder. The resected specimen exhibits a large multilobulated mass with a botryoid surface and a narrow attachment to the bladder mucosa. Microscopically, the lesion is composed of Brunn nest and cystitis cystica et glandularis. The stroma contained scattered benign fibroblasts, myofibroblasts, and smooth muscle cells and is strongly reactive for desmin, smooth muscle actin, $\mathrm{H}$-Caldesmon but negative for myogenin and ALK. Diagnosis of Botryoid Fibroepithelial Polyp was made. The child had an uneventful recovery postoperatively. FEPs of the urinary bladder are extremely rare. To the best of our knowledge, only three cases of Botryoid variant of FEP have been published in the English literature (two cases occur in children of 3 and 11 years old, another one occurs in adults of 40). Haematuria is the most common symptom. The main differential diagnosis includes rhabdomyosarcoma, polypoid cystitis, eosinophilic cystitis and inflammatory myofibroblastic tumour. A panel of immunohistochemistry are essential to establish the diagnosis. The pathogenesis of bladder FEPs remains unclear, though genetic mutation was proposed in few studies. Regardless of the aetiology, recurrence is rare, however, malignant transformation has been reported. Due to the rarity of this entity, FEP should be kept in mind as one of the differential diagnoses of bladder mass in children who present with haematuria and obstructive uropathy. The prognosis is good, and long-term followup and regular cystoscopy are highly suggestive. 


\title{
POSTER PRESENTATION
}

\section{ID97 PP066}

\section{Oral Contrast for Magnetic Resonance Enterography: Comparison Between Pineapple Juice and Different Mannitol Concentration}

\author{
Zulkifle, Ahmad Farid'; ${ }^{1}$ Abdul Hamid, Hamzaini².
}

${ }^{1}$ Department of Medicine, Faculty of Medicine and Health Science, Universiti Sains Islam Malaysia, Negeri Sembilan, Malaysia

${ }^{2}$ Department of Radiology, Faculty of Medicine, National university of Malaysia, Kuala Lumpur, Malaysia

A proper degree of bowel distension is important for adequate imaging of the small intestine as abnormalities may be missed in collapsed bowel segments. Volumen ${ }^{\circledR}$; the current most frequently used oral contrast agent, however in Malaysia, is not obtainable anymore. Therefore, the search for other alternative oral contrast agents are highly demanded. The study aimed to compare different oral contrast agents used in magnetic resonance enterography (MRE) in terms of small bowel distension, image quality, patient's acceptance and side effects. Seventy five MRE performed in the Radiology Department, Universiti Kebangsaan Malaysia Medical Centre were included in this study. Three different oral contrast solutions were used, namely, 6.7\% \& 3.3\% mannitol concentrations and pineapple juice. Using a systematic sampling method, each of the patients received one type of oral contrast agent. The small bowel distention, image quality, patient's acceptance and side effects were measured by one observer. Comparison between all 3 solutions were made using 2way ANOVA, and a comparison between 2 solutions were made using T-Test. There were significant differences in terms of bowel distension, image quality, patient's acceptance and side effects between all three solutions. For bowel distension and image quality, mannitol solutions were superior to pineapple juice, however no significant difference between them. Pineapple juice is the most favourable solution with lesser side effects, whereas $6.7 \%$ mannitol solution is the least favourable solution with the most incidence of side effects. Small bowel distension and image quality were better with mannitol than pineapple juice. In between two mannitol concentrations, 3.3\% mannitol concentration has better acceptance and lesser side effects thus making it a better choice as an oral contrast for MRE. It is also cheap and easier to get in the hospital setting. Mannitol 3.3\% concentration should be preferred as endoluminal contrast agent for bowel in MRE. 


\title{
POSTER PRESENTATION
}

\section{ID98 PP067}

\section{Critical Value Notification in a Chemical Pathology Laboratory of Tertiary Teaching Hospital: An Analysis}

\author{
Wan Norlina, Wan Azman ${ }^{1,2}$; Aniza, Mohammed Jelani ${ }^{1,2}$; Julia, Omar ${ }^{1,2}$; Noorazliyana, Shafii ${ }^{1,2}$; Nor Azlin \\ Azraini, Che Soh ${ }^{1,2}$; Tuan Salwani, Tuan Ismail ${ }^{1,2}$; Nur Karyatee, Kassim ${ }^{1,4}$; Hanim Afzan, Ibrahim ${ }^{1,4}$; Ahamd \\ Ezam, Zainan ${ }^{1,2}$; Wan Mohd Faizal, Wan Azman ${ }^{2,3}$. \\ ${ }^{1}$ Department of Chemical Pathology, School of Medical Science, Health Campus, Universiti Sains Malaysia, \\ Kubang Kerian, Kelantan, Malaysia \\ ${ }^{2}$ Hospital Universiti Sains Malaysia, Health Campus, Universiti Sains Malaysia, Kubang Kerian, Kelantan, \\ Malaysia \\ ${ }^{3}$ The Center for Knowledge, Communication and Technology, Health Campus, Universiti Sains Malaysia, \\ Kubang Kerian, Kelantan, Malaysia \\ ${ }^{4}$ School of Dental Sciences, Health Campus, Universiti Sains Malaysia, Kubang Kerian, Kelantan, Malaysia
}

Notification of critical values is essential for clinical laboratories to ensure patient safety. The study aimed to assess the frequency and the turnaround time(TAT) of critical value reporting for plasma ammonia, serum sodium( $(\mathrm{Na}+)$, total bilirubin(TB), potassium $\left(\mathrm{K}_{+}\right)$and calcium( $\left(\mathrm{Ca}^{+}\right)$in a different clinical care setting. This retrospective study was conducted in the Chemical Pathology Laboratory, Hospital Universiti Sains Malaysia, using Laboratory Information System data from January to December 2020. Based on Ministry of Health Malaysia guidelines, the critical values are defined as : plasma ammonia $>100 \mu \mathrm{mol} / \mathrm{L}$, serum $\mathrm{Na}+(<125 \mathrm{mmol} / \mathrm{L},>155 \mathrm{mmol} / \mathrm{L}), \quad \mathrm{K}+$ $(<2.8 \mathrm{mmol} / \mathrm{L},>6.0 \mathrm{mmol} / \mathrm{L})$, paediatric TB $>300 \mu \mathrm{mol} / \mathrm{L}$, and $\mathrm{Ca}+(<1.5 \mathrm{mmol} / \mathrm{L},>3.0 \mathrm{mmol} / \mathrm{L})$, The area of clinical care included emergency departments, outpatient clinics, critical, medical, surgical and paediatric wards. The TAT was reported based on $<30$ minutes and $>30$ minutes notifications. The total test request in 2020 was 611343 . The frequency and percentage $n(\%)$ of critical values notification in 2020 were 7752(1.27) out of the total test request. The serum $\mathrm{K}+$ contributed the highest critical value notification with $\mathrm{n}(\%)$ of $3968(51.19 \%)$ to the total critical values. From these percentages, the $\mathrm{n}(\%)$ of low and high serum $\mathrm{K}$, $2520(63.51)$ and $1448(36.49 \%)$, respectively. The least critical value notification was ammonia, with $\mathrm{n}(\%)$ of $35(0.45)$. The $\mathrm{n}(\%)$ of other analytes critical value notifications, $\mathrm{Na}+\mathrm{TB}$ and $\mathrm{Ca}+$, were 3124(40.30), 107(1.38) and 518(6.68), respectively. The highest percentage of critical values reported were from the critical wards, followed by the medical wards, $29 \%$ and $20.94 \%$, respectively, to the total critical values. The $\mathrm{n}(\%)$ notification of critical values $<30$ minutes and > 30minutes were $6711(86.57)$ and 1041(13.43) respectively to the total critical values. The most common analytes reported $<30$ minutes was $\mathrm{K}+$ with $\mathrm{n}(\%)$ of 3495(45.09). The most prevalent critical value analyte was serum potassium. Principally, critical values were reported $<30$ minutes, and the highest notification originated from the critical wards of this hospital. 


\title{
POSTER PRESENTATION
}

\section{ID99 PP068}

\section{Kelantan Multi-Disaster Risk Assessment and Best Practice During Pandemic}

Abdul Ghafar, Muhammad Ariffin ${ }^{1,2}$; Abdul Jalal, Aishah Sabrina ${ }^{1,3}$; Yahya, Nor Yazjehan ${ }^{1,4}$; Shaikh Mohd Salleh, Siti Nurliyana ${ }^{1}$; Razak, Khamarrul Azahari ${ }^{1}$.

\author{
${ }^{1}$ Malaysia-Japan International Institute of Technology, Universiti Teknologi Malaysia, Kuala Lumpur, Malaysia \\ ${ }^{2}$ Department of Emergency and Trauma, Hospital Shah Alam, Selangor, Malaysia \\ ${ }^{3}$ Program Development and Operations MERCY Malaysia, Kuala Lumpur, Malaysia \\ ${ }^{4}$ Department of Emergency and Trauma, Hospital Sultan Ismail, Johor Bahru, Johor, Malaysia
}

Floods were reported in Malaysia because of heavy rains in early 2021. The disaster has impacted 6,241 families and 23,776 persons. Kelantan is unexceptional from the inundation crisis during pandemic COVID-19. The multi-hazard disaster had brought serious attention among stakeholders from the rising pandemic cases during flood events. The purpose of this study is to investigate multi-challenges of pandemic, extreme weather, and climate change in Kelantan with best practices to manage multi-hazard which can be implemented to the future planning based on geohazard information for disaster risk assessment. Qualitative research method is adopted to conduct the research. The data collection was based on literature review and case study from Kelantan, Malaysia, and other best practice from other regions. Kelantan, a flood prone state facing challenges in implementing COVID-19 SOPs while optimizing healthcare delivery against multi-disaster. Three case studies covered by using application of GIS technologies and modern earth observation system within three multi-challenges of multi-disaster mainly pandemic, landslides, and floods in addressing the needs and demands of risk-informed sustainable development such as the satellite, Al, UAV, IoT and telemedicine. In conclusion, by incorporate the geospatial technologies advancement that align with global targets SFDRR, SDGs together with national and state policies such as GP 030 - Bandar Berdaya Tahan Bencana di Malaysia and RSN Kelantan 2020 will help to enhance the government and other stakeholders of Malaysia to be more resilient towards the future risk. 


\title{
POSTER PRESENTATION
}

\section{ID101 PP069}

\section{Sinonasal Malignant Melanocytic Melanoma as a Rare Cause of Epistaxis}

\author{
Mohamad, Adam'; Saravannan, Pavitra'; Yusuf, Suhaimi'; Abdul Wahab, Azwan Halim; Mohd Rajet, Khairul \\ Azhar'; Mohamad, Irfan², Ramli; Ramiza Ramza². \\ ${ }^{1}$ Department of Otorhinolaryngology, Head and Neck Surgery, Hospital Tengku Ampuan Afzan, Kuantan, \\ Pahang, Malaysia \\ ${ }^{2}$ Department of Otorhinolaryngology, Head and Neck Surgery, Universiti Sains Malaysia, Kubang Kerian, \\ Kelantan, Malaysia \\ ${ }^{3}$ Department of Otorhinolaryngology, Head and Neck Surgery, International Islamic University Malaysia, \\ Kuantan, Malaysia
}

Malignant melanocytic melanoma involving nasal cavity is a very rare condition. Nasal obstruction and epistaxis are the most commonly reported symptoms, although symptomatology may develop late and nonspecific. This report describes an elderly man with underlying dementia, who presented with epistaxis and progressive nasal blockage. Clinical examination revealed a blackish fungating lesion which was biopsied and removed under anesthesia. Histopathology examination suggested a sinonasal melanocytic malignant melanoma due to the strong and diffuse positivity for S100, HMB45, and Melan-A. Subsequent computed tomography of brain, neck, abdomen and pelvis revealed lung metastasis. He was referred to the oncology team for further treatment. Primary mucosal sinonasal melanoma corresponds to $0.5-2 \%$ of all malignant melanomas and approximately $4 \%$ of melanomas of the head and neck. Etiology, pathogenesis and specific risk factors remains unclear; however, mucosal melanomas are known to develop from melanocytes present in mucosal tissue originating from the migration of cells from the neural crest during the embryonic period. Radiotherapy did not improve local control and chemotherapy has not shown much advantage as compare to surgical resection. The case report discusses the distinct clinico-pathologic presentation as well as the need to comprehend its biological behavior for a more successful treatment outcome. 


\section{POSTER PRESENTATION}

\section{ID102 PP070}

\section{Study of Hematocrit Level in Obstructive Sleep Apnea Syndrome}

Jamaluddin, Jamal Sazly¹; Mohamad, Hazama²; Ramli, Ramiza Ramza².

${ }^{1}$ Department of Otorhinolaryngology, Hospital Bukit Mertajam, Pulau Pinang, Malaysia.

${ }^{2}$ Department of Otorhinolaryngology, Head and Neck Surgery, Universiti Sains Malaysia, Kota Bharu, Kelantan, Malaysia.

Obstructive Sleep Apnea Syndrome (OSAS) can lead to oxyhemoglobin desaturation and has the possibility to stimulate erythropoiesis leading to increased hematocrit. The aim of this study is to identify the level of hematocrit in OSAS patients and to determine the correlation between hematocrit levels and severity of OSAS. This is a descriptive cross-sectional study in Hospital Universiti Sains Malaysia. All consented patients were interviewed and underwent polysomnography (PSG) study. The severity of OSAS was based on Apnea-Hypopnea Index (AHI) and average oxygen desaturation. Blood hematocrit level was also obtained. Thirty-six OSAS patients were in the study group and 17 patients in the control group. Mean hematocrit level in the control group was lower $(41.6 \pm 2.5)$ as compared to the study group $(44.0 \pm 3.27)$. Pearson correlation analysis showed no significant correlation between $\mathrm{AHI}$ and hematocrit level $(p=0.392)$. There is a significant correlation between hematocrit levels with average oxygen saturation level $(p<0.05)$. The independent $\mathrm{t}$-test between mean hematocrit level and OSAS group versus control group showed a significant difference $(p<0.05)$. The increased level of hematocrit is significantly correlated with average oxygen desaturation but not with AHI in OSAS patients. Average oxygen desaturation can be considered as a parameter to assess severity and hypoxic stress in OSA patients. 


\title{
POSTER PRESENTATION
}

\section{ID103 PP071}

\section{Knowledge, Attitude and Practice Towards Sharp Injuries Prevention Among Nurses}

\author{
Seman, Nordianna ${ }^{1,2} ;$ Albert, Jennifer ${ }^{2}$. \\ ${ }^{1}$ Center for Nursing Studies, Faculty of Health Sciences, University of Technology MARA, Puncak Alam, \\ Selangor, Malaysia \\ ${ }^{2}$ Open University Malaysia, Seberang Jaya, Penang, Malaysia
}

The nursing working environment can be described as a high-risk zone due to numerous hazards including sharp injuries. Globally, the occurrences of this common hazard have affected both the physical and mental well-being of nurses. Based on this concern, we explored the knowledge, attitude, and practice towards sharp injuries prevention among registered nurses. Our specific study objectives include (1) To identify the knowledge level regarding prevention of sharp injuries among registered nurses, (2) To determine the attitudes of registered nurses towards prevention of sharp injuries, (3) To identify the extent of practices in which registered nurses adhere and comply with safety precautions in preventing sharp injuries, and (4) To distinguish the significant relationship between knowledge, attitude and practice among senior and junior registered nurses towards prevention of sharp injuries in a private hospital. We employed the quantitative study design with a cross-sectional survey and this study was conducted in a private hospital in Penang, Malaysia. Registered nurses were recruited by purposive sampling $(\mathrm{N}=103)$. The result of the study discovered that a good knowledge level with a positive attitude towards sharp injuries. However, the mean practice score towards sharp injuries prevention was found to be moderate $(3.05 \pm 0.24)$. There were significant differences observed in knowledge and practice towards the prevention of sharp injuries between senior and junior nurses $(p<0.05)$. Although this study indicates that the registered nurses are knowledgeable and have positive attitudes with moderate practice, they need to learn and adhere to the protocol by sharpening their theoretical knowledge and practical skills to achieve a better outcome towards the prevention of sharp injuries occurrence. Thus, all registered nurses are encouraged and should be kept abreast with the current changing trends concerning sharp injuries prevention. 


\title{
POSTER PRESENTATION
}

\section{ID104 PP072}

\section{Parathyroid Adenoma in a Patient with Renal Cell Carcinoma: A Rare Association}

Kassim,Nur Karyatee ${ }^{1,2,4} ;$ Yahya,Maya Mazuwin ${ }^{3,4}$;Tuan Ismail,Tuan Salwani ${ }^{2,4}$;Thien Jun, Jun ${ }^{5}$; Mohamad, $\operatorname{Irfan}^{4,6}$.

\author{
${ }^{1}$ School of Dental Sciences, Universiti Sains Malaysia, Kubang Kerian, 16150 Kelantan, Malaysia \\ ${ }^{2}$ Department of Chemical Pathology, School of Medical Sciences, Universiti Sains Malaysia, Kubang Kerian, \\ 16150 Kelantan, Malaysia \\ ${ }^{3}$ Department of Surgery, School of Medical Sciences, Universiti Sains Malaysia, Kubang Kerian, 16150 \\ Kelantan, Malaysia \\ ${ }^{4}$ Hospital Universiti Sains Malaysia, Kubang Kerian, 16150 Kelantan, Malaysia \\ ${ }^{5}$ Department of Pathology, Sarawak General Hospital, Jalan Hospital,93586, Kuching Sarawak, Malaysia \\ ${ }^{6}$ Department of Otorhinolaryngology-Head \& Neck Surgery, School of Medical Sciences, Universiti Sains \\ Malaysia, Kubang Kerian, 16150 Kelantan, Malaysia
}

The coexistence of renal cell carcinoma (RCC) and primary hyperparathyroidism is a rare occurrence. Hypercalcemia in the presence of malignancy is commonly attributed to paraneoplastic phenomenon, particularly when associated with RCC. However, other causes of hypercalcemia may coexist and be treatable. We present the case of a 58-year-old male who was detected to have a left abdominal mass during a routine medical follow-up and was later diagnosed with RCC. Further assessment revealed that his calcium levels had been persistently high for eight years prior to the presentation but had been managed conservatively as he was asymptomatic. Based on high calcium and parathyroid hormone (PTH) level as well as radiological findings, a diagnosis of primary hyperparathyroidism was made. A right inferior parathyroidectomy was performed, and the histopathological examination results showed right parathyroid adenoma. Intraoperative intact PTH measurements confirmed the complete removal of the abnormal parathyroid gland. The post-operative calcium levels resumed to normal value. To the best of our knowledge, this is the first reported case of a patient with concurrent primary hyperparathyroidism and RCC in our population. Broad differential diagnoses should be considered when evaluating patients with hypercalcemia. 


\title{
POSTER PRESENTATION
}

\section{ID106 PP073}

\section{Undetectable HbA1c Level in a Diabetic Patient with Leukaemia}

\author{
Wan Nik, Wan Nor Fazila Hafizan ${ }^{1,2}$; Tuan Ismail, Tuan Salwani ${ }^{1,2}$; Kassim, Nur Karyatee ${ }^{3}$ \\ ${ }^{1}$ Department of Chemical Pathology, School of Medical Sciences, Universiti Sains Malaysia, Kubang Kerian, \\ 16150 Kelantan, Malaysia \\ ${ }^{2}$ Hospital Universiti Sains Malaysia, Kubang Kerian, 16150 Kelantan, Malaysia \\ ${ }^{3}$ School of Dental Sciences, Universiti Sains Malaysia, Kubang Kerian, 16150 Kelantan, Malaysia
}

In a cancer patient with underlying diabetes, good glycemic control should be individually managed to ensure the patient has no hyperglycemia symptoms either develop iatrogenically due to anti-cancer therapy or relative improper diabetic control by the patient. HbA1c is considered a good marker of glycemic control but it is susceptible to some interference. We present a case of 45-year-old man with underlying diabetes for 12 years, who was diagnosed as T-cell prolymphocytic leukemia (T-PLL) with hyperleukocytosis in view of raised white cell count at 614.60 x 10,/L (lymphocytes predominant: $547.21 \times 10 \% / L)$. He was started on chemotherapy and tumor lysis syndrome prophylaxis. His insulatard continued as prescribed. There was an absent HbA1c peak on the initial run using capillary electrophoresis which did not correlate with his FBS of $12.6 \mathrm{mmol} / \mathrm{L}$. The sample was then treated by washing with saline and analysis was repeated. Post treatment sample was improved by a well fractionated $\mathrm{HbA} 1 \mathrm{c}$ peak with a value of $8.7 \%(71 \mathrm{mmol} / \mathrm{mol})$. The exact mechanism of no HbA1c peak in the patient's sample is unknown. Our postulation is due to accelerated migration speed of the sample causing a shift of the profile that may result in a non-recognition of the fractions. It is postulated that the interference might come from molecules produced by the white cells not by the cells themselves since the washing of the cells eliminates the migration shift in most of the cases. This may be observed in some cases of patients with hyperleukocytosis similarly in this case. The recovery of HbA1c peak after washing step implies that some molecules in the plasma are responsible for the assay interference during analytical process. 


\section{POSTER PRESENTATION}

\section{ID107 PP074}

\section{Potential Protective Effects of Tualang Honey on Chronic Combinatory Stressed-rat Testis}

Mohd Amin, Mohamad Syabil Ikhwan ${ }^{1}$; Mohd Yusof, Nurul Aiman'; Norman, Haziq Hazman²; Asari, Mohd Asnizam $^{1}$.

${ }^{1}$ Department of Anatomy, School of Medical Sciences, Universiti Sains Malaysia, 16150 Kubang Kerian, Kelantan, Malaysia.

${ }^{2}$ Anatomy Unit, Management and Science University (MSU), University Drive, Off Persiaran Olahraga, Section 13, 40100 Shah Alam, Selangor, Malaysia.

Prolonged exposure to chronic stress has been shown to cause negative effects on testicular reproductive functions. Honey has been shown to ameliorate the adverse effects caused by chronic combinatory stress [i.e., restraint stress (RS) + forced swimming test (FST)]. This study aimed to investigate the potential protective effects of Tualang honey against stress-induced testicular damage in male rats. Twenty-four male Sprague-Dawley rats were divided into four groups: control, stress-exposed, Tualang honey supplemented control group ( $1.0 \mathrm{~g} / \mathrm{kg}$ body weight) and stress-exposed supplemented with Tualang honey $(1.0 \mathrm{~g} / \mathrm{kg}$ body weight). The stress procedure involved a combination of RS and FST for 21 days. Serum was collected to measure the testosterone concentration using ELISA. Testis were harvested, processed, and stained by using haematoxylin and eosin (H\&E) for spermatid counts and measuring seminiferous epithelial height. One-way analysis of variance (ANOVA) was used to compare the numerical data between groups. The testosterone concentration, spermatid count and epithelial height in the stress group were significantly lower compared to the control group $(p<0.05)$. The spermatid count and epithelial height of honey-treated groups were significantly higher compared to stress-only groups $(p<0.05)$. However, the testosterone concentration of honey-treated groups was higher compared to the stress-only group despite insignificant results. To the best of our knowledge this study is the first to show the potential protective outcomes of Tualang honey in attenuating effects of chronic combinatory stress on testosterone concentration, spermatid count, and epithelial height of testicular seminiferous tubules. 


\section{POSTER PRESENTATION}

\section{ID108 PP075}

\section{Survey on Exercise-Related Leg Pain (ERLP) among University Recreational Runners}

Latir Aliff ${ }^{1}$; Bakhtiar Jamily Ahmed Ali Bashir ${ }^{1}$

${ }^{1}$ Centre of Physiotherapy, Faculty of Health Sciences, Universiti Teknologi Mara (UiTM) Cawangan Selangor, Kampus Puncak Alam, 42300 Bandar Puncak Alam, Selangor, Malaysia.

Exercise-related leg pain (ERLP) is a common overuse injury with an incidence of 27$33 \%$ among runners and military personnel. ERLP is associated with internal and external risk factors such as gender, BMI, ERLP history, running experience and cross training. However, little is known regarding ERLP risk factors and its effects on activities of daily living in university recreational runners. This study aimed (i) to report the prevalence of ERLP; (ii) to determine the effects of ERLP on daily activities; and (iii) to investigate the relationship between the potential risk factors and ERLP among university recreational runners. This is a cross-sectional, observational study which recruited 159 recreational runners in UiTM Puncak Alam campus through a purposive sampling method. An online survey consisting of demographic and ERLP questionnaire adopted from Reinking et al. (2010) was distributed amongst the UiTM Runners Club (URC) and fan Facebook page. SPSS v. 21.0 was used to analyse the relevant data. Chi-square was used to determine the association of the risk factors with ERLP. P-value is set at 0.05 . Majority $(86.80 \%)$ of the university recreational runners reported incidence of ERLP. Among runners with ERLP, $63.80 \%$ had to reduce their running mileage in training, $14.50 \%$ had to stop running, $2.20 \%$ had to stop walking and $0.70 \%$ unable to manage stairs, respectively. Moreover, ERLP history was significantly associated with the incidence of ERLP $(p<0.05)$. ERLP prevalence in university recreational runners is high. Most runners affected by ERLP had to reduce their running mileage but not severely affecting other daily activities such as walking and stairs climbing. The only risk factor contributing to ERLP among the affected runners is ERLP history. Further investigation is warranted to identify other risk factors that may occur singularly or combined in a larger running population. 


\title{
POSTER PRESENTATION
}

\section{ID110 PP077}

\section{The Knowledge, Attitude, and Practice of Physical Activity Among Community Dwelling Stroke Survivors in Klang Valley, Malaysia}

\author{
Ong Ke Yee ${ }^{1}$; Lian Sin Jie ${ }^{1}$; Haniz Naqib bin Hazaidi ${ }^{1}$ : Nor Azlin Mohd Nordin ${ }^{1}$. \\ ${ }^{1}$ Physiotherapy Program, Faculty of Health Sciences, Universiti Kebangsaan Malaysia (UKM), Kuala Lumpur, \\ Malaysia.
}

Stroke is a major cause of functional disability in adults worldwide. Literature has documented that physical activity (PA) is likely to reduce the disability related to stroke and the risk of further stroke. The practice of PA is strongly influenced by knowledge of and attitude towards PA. Previous studies used the Stroke Knowledge Test (SKT) questionnaire to assess knowledge of PA. However, to date no studies have been conducted to evaluate the knowledge, attitude, and practice (KAP) of PA neither among the stroke population nor the stroke survivors. To determine the Knowledge, Attitude, and Practice of PA among stroke survivors. 179 stroke survivors were recruited using a simple random sampling method. A survey questionnaire was adapted from the Stroke Knowledge Test (SKT), Stroke Physical Activity Questionnaire (SPAQ) and KAP of physical activity questionnaires for a healthy population which tested for validity and reliability. Participants answered the structured questionnaire manually or via a phone call with guidance from a physiotherapy student. Data were entered and analyzed using SPSS $\vee 26$. The participants' response rate was good (89\%). Educational status was statistically significant to the level of knowledge $(p=0.02)$. Participants have a fair to good knowledge level and $91.1 \%(n=163)$ have a positive attitude toward PA. $64.8 \%$ $(n=116)$ were physically inactive. The top 3 main barriers were patient-related factors, fear of falls and pandemic. A low correlation was found between knowledge and practice of PA $(r=+0.220, p<0.001)$. Generally, although stroke survivors have a fair to good knowledge level and a positive attitude toward PA, their PA practice is low. This is correlated to the barriers explored and illustrated by a sedentary lifestyle. Strategies should be outlined to overcome barriers and enhance PA levels among stroke survivors. 


\title{
POSTER PRESENTATION
}

\section{ID111 PP078}

\section{Blended Clinical Learning for Undergraduate Paediatric Rotation Post-Pandemic: The USIM Experience}

\author{
Rusli, Nur Sabrina ${ }^{1}$; Mohammad Khuzaini, Aliyyah ${ }^{1}$; Abdul Halim, Halimah ${ }^{1}$; Maimun Aqsha, Nur Syahirah ${ }^{1}$; \\ Suhaidi, Siti Sarah Aishah'.
}

${ }^{1}$ Paediatric Unit, Faculty of Medicine and Medical Sciences, Universiti Sains Islam Malaysia, Nilai, Malaysia

Over the past decades, pandemics caused by viruses have disrupted many normal human activities. The current Covid pandemic caused by SARS-CoV-2 poses a special challenge, being very contagious and easily transmissible through the. This precludes close contacts that is the hallmark of clinical experiential teaching. A key challenge in medical education is therefore the provision of clinical teaching outside the conventional hospital-based learning with hospitals being made inaccessible to students and teachers. This report describes the adoption of a blended learning program into the paediatric clinical rotation in lieu of the experiential hospitalbased rotation. This approach comprised of history simulation sessions, communication workshops, clinical signs and symptoms plenary and virtual ward rounds. Principles of adult learning theories and self-directed learning were applied throughout the rotation. Adult learning theory encompassed concepts of ready to learn, intrinsic motivation, learning through experience, self-direction, problemoriented, deliberate practice, self-directed learning, flipped classroom and collaborative learning. The concepts are applied to develop skills in history taking, physical examination, developing differential diagnoses, planning for investigations, and finally communicating the findings. For history (and Differential Diagnoses)" tasks, the Jamboard "Questions to ask" approach is adopted with students also provided with pre-reading materials. These are complemented with classroom history simulation and post-classroom "Approach to" posters. For skills in physical examination (and Differential Diagnoses) there will be pre-classroom work involving the Wikilibrary and examination videos, classroom work involving signs and symptoms, plenary, and virtual ward round. Post-classroom exercises will include Approach to "posters". The blended learning programme is a viable alternative to hands-on clinical rotation during the pandemic. It is expected that results will help inform on alternative approaches to clinical skills development among medical students with the application of information technology to meet the threats of future pandemics. 


\title{
POSTER PRESENTATION
}

\section{ID113 PP079}

\section{The Design of a Socially Intelligent Agent with Empathy for Mental Health Support}

\author{
Mohamad Farif Jemili ${ }^{1}$, Azizi Ab Aziz ${ }^{1}$ \\ ${ }^{1}$ Relational Machines Group, Human-Centred Computing Lab, School of Computing, Universiti Utara Malaysia
}

Socially Intelligent Agents (SIAs) can be described as digital entities that can engage in social interaction with humans, using both verbal and non-verbal modalities. These agents can express/perceive emotions, communicate with high-level dialogue, exhibit distinctive personality and character, as well as learning and developing social competencies. Examples of SIAs are social robots, virtual humans or interactive chatbots. SIAs have been introduced in mental healthcare domains, from screening to supporting on-demand therapy. However, some people do not experience much satisfaction from interacting with SIAs over more extended periods. They feel the SIAs do not really 'understand' or 'empathize' them as humans did, hence limiting its effectiveness. Therefore, the critical challenge is how to ensure that people experience more 'empathy' from SIAs. Hence, a new computational empathy to leverage SIAs potential in mental health support is needed. The main objective of this project is to develop an integrated computational empathy model for SIAs. Analysis of components related to empathy, social interaction and cognitive therapy were carried out. Subsequently, this model will be evaluated using formal verification and human experiment validation approaches. This is an ongoing project. The design was completed with the development of four main components (e.g., input module, personality evaluation, emotion and cognitive analysis). The input module covers the touch-enabled screen, speech, and vision recognition. The personality evaluation module measures experienced events, mental health status and traits. The emotion analysis module relates to the evaluation of real-time emotion status. The cognitive analysis covers the computationally generated empathy for SIAs. This paper explores the ongoing work. The initial result produces four components to be integrated with SIAs, as a platform to support an individual with mental health issues. Next, the deployment of those components with SIAs for human experiments will be conducted. 


\title{
POSTER PRESENTATION
}

\section{ID116 PP081}

\section{Medical Waste Disposal Management: An Investigation on Awareness, Knowledge and Practices of Public}

\author{
Nordin, Norshahrizan'; Ishak, Najwahanim'; Che Razak, Razli2; Shaharuddin, Muhd Ikram Fikrie'; Ramdzan, \\ Abdul Rahman; ; Saadon, Mohd Saiful Izwaan* \\ Healthcare Management - Special Interest Group (HEm - SIG), Management of Technology and Engineering \\ Management - Special Interest Group (MoTEm - SIG), Faculty of Applied and Human Sciences, Universiti \\ Malaysia Perlis, Kangar, Perlis, Malaysia \\ ${ }^{2}$ Faculty of Business and Entrepreneurship, Universiti Malaysia Kelantan, Bachok, Kelantan, Malaysia \\ ${ }^{3}$ Faculty of Medicine and Health Sciences, Universiti Malaysia Sabah, Sabah, Malaysia \\ ${ }^{4}$ Faculty of Maritime Studies, Universiti Malaysia Terengganu, Terengganu, Malaysia
}

The increasing number of health care institutions will increase the amount of medical waste generated. There is still a lack of public awareness about this issue, which can contribute to pollution which can contribute to health problems and also to the surrounding environment. This research paper is intended to study the level of public awareness on the issues of medical waste that might cause health problems. In addition, this study also aimed to test the knowledge of the public and also practical in the handling of medical waste. The assessment process is supported by the distribution of questionnaires to 100 members of the public in the state of Perlis as respondents. Descriptive statistics and multiple regression were used in data analysis. The respondents completed a self-administered questionnaire, and the study revealed the coefficient of R2 is 0.160 and 0.134 for adjusted R2. It is important to clarify the coefficient of R2 because it represents a relationship between independent variables and dependent variables. This means predictors (independent variables; awareness, knowledge, and practices) represent $13.4 \%$ change in medical waste disposal management and remaining $86.6 \%$ can be contributed by other factors that are not studied as this was beyond the scope of the study. However, as a general role, this model is considering not a good fit as the adjusted R2 is less than $60 \%$. The results showed that the general public is aware of the dangers that come from medical waste but there is still a lack of knowledge about that particular waste and are not practicing the proper way to handle it. In seriousness of medical waste disposal issues, respondents should give more attention and make aware of such situations rather than taking it lightly. 


\title{
POSTER PRESENTATION
}

\section{ID118 PP083}

\section{A Critical Incident Technique (CIT) Measurement for Analysis of Complaints and Compliments among Hospital Patients: A Case Study in Northern Region, Malaysia}

\author{
Nordin, Norshahrizan ${ }^{1}$; Anuar, Siti Athirah ${ }^{1}$; Che Razak, Razli²; Shaharuddin, Muhd Ikram Fikrie ${ }^{1}$; Saadon, \\ Mohd Saiful Izwaan ${ }^{3}$ \\ ${ }^{1}$ Healthcare Management - Special Interest Group (HEm - SIG), Management of Technology and Engineering \\ Management - Special Interest Group (MoTEm - SIG), Faculty of Applied and Human Sciences, Universiti \\ Malaysia Perlis, Kangar, Perlis, Malaysia \\ ${ }^{2}$ Faculty of Business and Entrepreneurship, Universiti Malaysia Kelantan, Bachok, Kelantan, Malaysia \\ ${ }^{3}$ Faculty of Maritime Studies, Universiti Malaysia Terengganu, Terengganu, Malaysia
}

This research was conducted to explore the determinants of the complaints and compliments among hospital patients. The questionnaire-based survey was carried out to investigate the level of communication, staff behaviors, waiting time and physical environment of towards quality improvement among hospital patients. Through complaints and compliments that have been voiced out by hospital patients, the Continuous Quality Improvement (CQI) for hospitals productivity will be more effective and efficient. Sampling location was in Northern Region. The analysis of Complaints and Compliments and Critical Incident Technique (CIT) provided a way for a better understanding for the patient's view of the services provided by the hospitals. The highest functional index was highest functional index is $\$ 24$ element $(0.8699)$ which indicates the patient's hospital will satisfied when the medical staff have an empathetic service attitude. Followed by $\mathrm{S} 10$ element $(0.8587)$ state the medical staff understands needs and requirements and lastly is S25 elements which is 0.8478 which means that nursing staff must has a skillful technique can cause the complaints and compliments among hospital patients. The findings of this research can be provided to the user, or the hospital management understands the critical factors that will cause complaints and compliments for hospital patients and proposing a suitable method to overcome this problem before it becomes worse. Evaluation of each determinant contributing to continuous quality improvement is needed for reducing the complaints to improve hospitals service in the future. Future research is warranted to provide a better understanding of other factors contributing to patient satisfaction with quality improvement in communication, staff behaviors, waiting time and physical environment and ways to improve them. 


\title{
POSTER PRESENTATION
}

\section{ID119 PP084}

\section{Postpartum Body Weight and Body Mass Index Changes Between Exclusive Breastfeeding and Non-Exclusive Breastfeeding Mothers of 6-Months Postpartum}

\author{
Balqis Sofeyya Binti Mohd Zawahid ${ }^{1}$, Farhanah Ahmad Shuhaimi ${ }^{1}$, Naleena Devi Muniandy ${ }^{1,2}$
}

${ }^{1}$ Centre of Nutrition and Dietetic, Faculty of Health Sciences, Universiti Teknologi MARA UiTM.

${ }^{2}$ Maternal, Infant and Young Child Nutrition (MiChild) Research Group, Universiti Teknologi MARA, Cawangan Selangor, 42300 Puncak Alam, Selangor, Malaysia

Failure to return to pre-pregnancy weight after gestation is one of the contributing factors to obesity that may impact women's health in the long-term. Hence, pregnant women are advised to gain weight according to recommendation, be physically active, reduce energy consumption and continue to exclusively breastfeed until 6 months to promote postpartum weight loss. Limited studies have reported the effect of breastfeeding on postpartum weight gain and body mass index (BMI) among Malaysian women. The objective of this study was to compare the differences in 6-months postpartum weight and BMI of exclusively and non-exclusively breastfeeding mothers. This cross-sectional study was conducted from August to November 2020. The study was conducted using an online survey via Google form which was composed of eight sections related to breastfeeding practices of mothers. The questionnaire was distributed to mothers through identified support groups on social media. 116 mothers who met the selection criteria were included in this study. The study indicated that $17 \%$ of the mothers were underweight, $53 \%$ had normal BMI, while 22\% were overweight and 9\% were obese before pregnant. Exclusively breastfeeding mothers showed decreasing trend in postpartum weight $(-1.04 \pm 4.66$ $\mathrm{kg})$ and $\mathrm{BMI}(0.34 \pm 2.31 \mathrm{~kg} / \mathrm{m} 2)$ while mothers who are not exclusively breastfeed showed an increase in weight gain $(0.32 \pm 4.25 \mathrm{~kg})$ and $\mathrm{BMI}(0.32 \pm 4.25 \mathrm{~kg} / \mathrm{m} 2$. $)$. However, there was no significant difference of postpartum weight and BMI changes between exclusively breastfeeding and non-exclusively breastfeeding mothers of 6months postpartum $(p>0.05)$. In conclusion, we found no significant difference of postpartum body weight and BMI changes among exclusively breastfeeding and nonexclusively breastfeeding mothers of 6-months postpartum. Future studies should be conducted for a longer period to monitor the differences in the trend in weight and $\mathrm{BMI}$ changes among breastfeeding and non-breastfeeding mothers. 


\title{
POSTER PRESENTATION
}

\section{ID120 PP085}

\section{Cortical Motor Potency and Muscle Plasticity Interplay Following Noninvasive Brain Stimulation in Ischaemic Stroke Patients}

\author{
Mah Hussain, Mohd Izzat Hakimi ${ }^{1}$; Hanafi, Muhammad Hafiz ${ }^{1}$; Mustapha, Muzaimi ${ }^{1}$; Kassim, Nur Karyatee ${ }^{2,3}$; Simok, \\ $\underline{\text { Anna Alicia }}$ \\ ${ }^{1}$ Department of Neurosciences, School of Medical Sciences, Health Campus, Universiti Sains Malaysia, Kubang \\ Kerian, 16150 Kota Bharu, Kelantan, Malaysia. \\ 2 Department of Chemical Pathology, School of Medical Sciences, Health Campus, Universiti Sains Malaysia, \\ Kubang Kerian, 16150 Kota Bharu, Kelantan, Malaysia. \\ ${ }^{3}$ School of Dental Sciences, Health Campus, Universiti Sains Malaysia, Kubang Kerian, 16150 Kota Bharu, \\ Kelantan, Malaysia. \\ ${ }^{4}$ Department of Anatomy, School of Medical Sciences, Health Campus, Universiti Sains Malaysia, Kubang \\ Kerian, 16150 Kota Bharu, Kelantan, Malaysia.
}

There is much interest in the present state of stroke and motor rehabilitation research within the neuroscience community. Despite the effectiveness of stroke rehabilitation services, conventional neurorehabilitation therapies primarily target muscles and peripheral nerves, with little impact on neuroplasticity. Furthermore, no previous research has investigated the effects of repetitive Transcranial Magnetic Stimulation (rTMS) on Creatine Kinase (CK) and Troponin T (Trop T) levels in ischaemic stroke patients. This study aimed to determine the effects of $10 \mathrm{~Hz}$ rTMS in treating lesional side primary motor cortex (M1) on serum muscle and cardiac biomarkers. This research enrolled sixteen patients who were randomly allocated into two groups. The intervention group received facilitatory $10-\mathrm{Hz}$ rTMS over the lesional hemisphere side combined with conventional physiotherapy. Each participant of the intervention group received a total of 10 rTMS sessions and physiotherapy over two weeks. The control group underwent ten sessions of conventional physiotherapy without any stimulation. Pre- and post-treatment blood samples were taken from both groups. The levels of $\mathrm{CK}$ and Trop T levels within the control and intervention groups were analysed using ARCHITECT 8000 analyzer. There was no significant difference in post-intervention CK level and Trop T levels between the control and intervention groups, $p=0.300$ and $p=0.540$, respectively. There were no significant changes in the $\mathrm{CK}$ within each group. This study also revealed no significant changes of Trop $T$ within the intervention group, $\mathrm{p}=0.208$. Interestingly, the control group showed significant improvement in the Trop T level, $p=0.028$. The present study concludes that ischaemic stroke patients who received high frequency rTMS in combination with conventional physiotherapy have similar biomarker levels to those who received conventional physiotherapy alone. There may be possible correlation between individual response and the rTMS combination regime. This study also introduced a new dimension of cardiac safety in rTMS. 


\title{
POSTER PRESENTATION
}

\section{ID121 PP086}

\section{Protecting our Elderly: Senior Care during COVID-19 crisis}

\author{
Aziz, Amira Aqila, ${ }^{1}$; A. Rashid, Mohd Radzniwan²; Mohd Manzor, Nur Fariha, Addnan, Faizul Helmi; Roslan, \\ Nuruliza ${ }^{3,4}$ \\ ${ }^{1}$ Faculty of Medicine and Health Science, University Science Islam Malaysia (USIM) \\ 2 Department of Primary Health Care, Faculty of Medicine and Health Science, University Science Islam \\ Malaysia (USIM) \\ ${ }^{3}$ Biochemistry Unit, Department of Medical Sciences 1, Faculty of Medicine and Health Science, University \\ Science Islam Malaysia (USIM) \\ ${ }^{4}$ Islamic Science Institute, Universiti Sains Islam Malaysia (USIM)
}

The issue of COVID-19 had overwhelmed the world with elderly being the vulnerable population to record the highest mortality cases. Geriatric population, due to many reasons, include pre-existing comorbidities such as hypertension, diabetes or cardiovascular disease making them immunocompromised which lead them to higher infection risk. To discuss the importance of care towards the rising issue on elderly population and its association as a high-risk group during COVID-19 pandemic. Literature review has been done by using online databases: PubMed, UpToDate, Google Scholar to retrieve information based on study relevance. The keywords used in the search include Coronavirus OR COVID-19 OR SARS-CoV-2 OR 2019-nCoV; elderly OR geriatric population OR older adult OR ageing OR senior citizens; and safety OR care OR wellbeing. 22 journal articles were found based on the search criteria. Immunosenescence and inflammaging occurs due to accumulation of proinflammatory cells and mediators which further causing cytokine storms becomes key pathogenic mechanisms for understanding severe COVID-19 infection among elderly population. Due to the COVID-19 pandemic, issues such as access to healthcare and support, mental health issues, misleading information and elderly abuse had posed a grave impact to the elderly community. Telemedicine is becoming more crucial during the pandemic for healthcare optimization and reducing the risk of disease transmission. Hence, early vaccination, frequent symptom screening and viral testing of COVID-19 as well as PPE usage can be applied for mass disease control especially in closed spaces like nursing homes. Authorities must establish better policy and management whereby the community should be in solidarity to ensure the safety and survival of geriatric population towards convalescence of COVID-19. 


\title{
POSTER PRESENTATION
}

\section{ID122 PP087}

\section{Attitudes, Beliefs and Perceived Barriers in Assessment of Sexual Health among Nursing Students}

\author{
Fatimah Sham ${ }^{1}$, Amira Safiah Saihuddin ${ }^{1}$, Frannelya Francis ${ }^{3}$, Siti Munirah Abdul Wahab ${ }^{2}$, Nordianna Seman ${ }^{1}$ \\ ${ }^{1}$ Faculty of Health Sciences, Puncak Alam Campus, Universiti Teknologi MARA (UiTM), 42300, Malaysia \\ ${ }^{2}$ Centre of Nursing, Faculty of Health Sciences, Universiti Teknologi MARA Penang Branch, 13200 Kepala Batas, \\ Penang, Malaysia \\ ${ }^{3}$ Faculty of Health Sciences, Universiti Teknologi MARA Sarawak Branch, 94300 Kota Samarahan, Sarawak, \\ Malaysia
}

Sexual health assessment is crucial in addressing patients' sexual health problems that comprises of physical, psychological, social, and moral aspects. It is considered essential in holistic nursing care provision. However, preliminary studies indicate that the conduct of sexual health assessment among nursing students remain isolated. We explored the attitudes, beliefs, and perceived barriers towards sexual health assessment among nursing students. In specific, the study objectives include 1) To determine the attitudes and beliefs towards sexual health among nursing students, 2) To identify the perceived barriers related to sexual health assessment in clinical nursing practice, and 3) To examine the relationship between attitudes and beliefs towards sexual health assessment with the demographic attributes. A cross-sectional study was conducted in three different UiTM campuses. We employed the convenience sampling approach on both undergraduate and postgraduate nursing students $(\mathrm{N}=312)$. A self-administered questionnaire was utilized comprising the Sexual Attitudes and Beliefs Survey (SABS) and Barriers to Sexual Health Assessment in Clinical Nursing Practice adopted from Erenel \& Ozdemir (2020). Negative attitudes toward sexual health were found in this study (44.58 \pm 5.83$)$. Interestingly, most of the respondents agreed that sexual health assessment is essential to patients' health outcomes $(90.1 \%)$ and most of them $(88.1 \%)$ understood that patients' diseases and treatment might affect their sexual health. However, $57.4 \%$ of respondents felt uncomfortable talking about sexual issues and they don't feel confident in their ability to discuss sexual health (51\%). We also found that the relationship between attitudes and beliefs with study site, education level, and year of study were statistically significant $(p<0.05)$. In conclusion, our study found negative attitudes and beliefs toward sexual health assessment. Perceived barriers have further impeded the respondents' ability to perform sexual health assessments and influence their personal's views regarding sexual health issues. 


\section{POSTER PRESENTATION}

\section{ID123 PP088}

\section{Royal Free Hospital Nutrition Priotizing Tools (RFH-NPT): Predictor of Malnutrion Risk Among Chronic Liver Disease Patients in Malaysia}

Norashimah Rajab ${ }^{1}$, Syahrul Bariah Abdul Hamid ${ }^{1 *}$ Khairil Anuar Md Isa², Aishah Hanum Said ${ }^{3}$

${ }^{1}$ Centre of Dietetics Studies, Faculty of Health Sciences, UiTM Puncak Alam, 42300 Selangor, Malaysia ${ }^{2}$ Centre of Basic Sciences Studies, Faculty of Health Sciences, UiTM Puncak Alam, 42300 Selangor, Malaysia

${ }^{3}$ Dietetic and Food Service Department, Hospital Selayang, 68100 Batu Caves, Selayang, Malaysia

Patients with hepatic diseases are highly prone to malnutrition because of the key role played by the liver in regulating nutritional state and energy balance. The Subjective Global Assessment (SGA) is known as the gold standard in assessing nutritional status worldwide. Meanwhile, the Royal Free Hospital-Nutritional Prioritizing Tool (RFH-NPT) is recommended by the European Society for Clinical Nutrition and Metabolism (ESPEN) guidelines to detect malnutrition risk in patients with liver disease. In Malaysia, however, little is known regarding the use of RFHNPT to predict malnutrition risk among liver disease patients. The aim of this study is to evaluate the association of RFH-NPT with malnutrition risk. A total of 80 chronic liver disease patients without liver cancer were enrolled in this prospective study. This study was conducted at a government hospital located in Selangor which is the national tertiary referral center for liver disease in Malaysia. We administered the nutrition screening of Nutritional Risk Screening 2002 (NRS-2002), RFH-NPT, and SGA to the patients within $24 \mathrm{~h}$ after admission. The RFH-NPT had higher sensitivity (82\%) and specificity (85\%) as compared to NRS 2002 in screening for the malnutrition risk. The SGA assessment identified malnutrition in $75 \%(95 \%$ $\mathrm{Cl}, 65 \%-80 \%)$ while $80 \%$ of patients $(95 \% \mathrm{Cl}, 60 \%-95 \%)$ identified as at risk of malnutrition using RFH-NPT. Further analysis revealed RFH-NPT had significant correlation with SGA assessment $\left(p=0.039, R^{2}=0.48\right)$, Body Mass Index (BMI) $\left(p=0.042, R^{2}=0.47\right)$ and Child Pugh Score (CPS) $\left(p=0.048, R^{2}=0.44\right)$. Incorporating the RFH-NPT as a routine nutrition screening protocol is highly beneficial in assisting early identification patients at risk of malnutrition among liver disease patients. Hence, immediate dietetic interventions can be administered, in a timely manner to avoid further complications. 


\section{POSTER PRESENTATION}

\section{ID124 PP089}

\section{Online Distance Learning for Clinical Nutrition Practice Courses}

Mazuin Kamarul Zaman ${ }^{1}$; Noor Izati Roslan ${ }^{1}$; Nurfarah Hanani Amir Hamzah ${ }^{1}$, Muhammad Hakim Abdul Jalil' Siti Fasihah Abdul Razak ${ }^{1}$, Siti Nor Fatihah Ismail ${ }^{1}$

${ }^{1}$ Centre of Dietetics Studies, Faculty of Health Sciences, UiTM Cawangan Selangor, Kampus Puncak Alam, 42200 Kuala Selangor, Selangor, Malaysia

COVID-19 pandemic normalizes online learning as the main alternative for teaching and learning activities. The challenges of online learning are more prominent for hands-on courses among medical and health sciences students. Traditionally, clinical nutrition practice courses (CNPC) s' students receive on-site (outpatient, inpatient, and special units) training in clinics and hospitals. However, the movement control order (MCO) implemented in Malaysia prevented students from going to health institutions. Recognizing this barrier, innovations in the mode of delivery of the CNPC were made. Nutrition care process (NCP) includes nutrition assessment, diagnosis, intervention, monitoring, and evaluation. Two main Online Distance Learning (ODL) methods used for NCP were online counseling with patients via Google Meet and mock case studies. A set of structured case studies was developed based on the syllabus and instructors will act as patients for students to assess. Students will be assigned to instructors who will supervise them throughout the course. As nutrition assessments are limited to online interviews, students were requested to prepare videos of them conducting nutrition-focused physical examinations and anthropometric measurements to be reviewed by instructors. Weekly interactive sessions were arranged between students and experts (clinical dietitians and products specialists) via an online meeting platform. Overall, all students managed to complete ODL for CNPCs. While acceptance to ODL varies among students, the progression of each student can be seen through improvements in their case management and case reports. Innovations in ODL for hands-on codes have allowed it to continue despite the current pandemic. The various approach needs to be made to ensure engagement and learning outcomes to be achieved ODL for CNPCs is feasible but not preferable compared to on-site training. Multimodal delivery is required to keep the students engaged and provide a more holistic experience for students. 


\section{POSTER PRESENTATION}

\section{ID126 PP090}

\section{Why are Children not affected by Severe or Fatal COVID-19? A Preliminary, Digital Systematic Review on Protective Immuno- Pathophysiological Mechanisms in the Paediatric Population}

Nor Aripin; Khairun Nain Bin ${ }^{1}$, Sani; Anuar ${ }^{1}$, A.Rashid; Mohd Radzniwan ${ }^{1}$, Addnan; Faizul Helmi ${ }^{1}$, Mansur; $^{2}$ Fadlul Azim Fauzi ${ }^{1}$, Baharom; Nizam ${ }^{1}$, Anuar; Khairullah ${ }^{1}$

${ }^{1}$ Faculty of Medicine and Health Sciences, Universiti Sains Islam Malaysia, Nilai, Malaysia

COVID-19 was declared a pandemic in March 2020. A prominent observation is the striking difference in age-related severity. Children are very rarely affected by severe or fatal COVID -19. A study of the population $<18$ years in England discovered only 25 deaths due to COVID -19, giving a death rate of 2 per million with $99.995 \%$ survival rate of COVID -19. As of 1st June, Malaysia recorded only 15 COVID -19 deaths in total among the population $<18$ years of age. Several factors have been proposed to explain the difference between children and adults in COVID -19 severity. The objective of this study is to explore researched mechanisms that protect the paediatric population against severe/fatal COVID -19. A preliminary digital systematic search was conducted using Pubmed. Keywords used were "COVID" (all fields), "child"” (all fields), "mechanism" (title/abstract) and were combined with Boolean operator AND. The resulting citations were screened for eligibility based on the abstract. The digital search yielded 195 citations. Several protective immunopathophysiological mechanisms were frequently cited. These include age-related differences in ACE2 expression and affinity, age-related increase in endothelial dysfunction, existing immunity to human coronaviruses (HCoVs), increased innate and weaker adaptive immune stimulation in children, greater anti-inflammatory and anti-oxidative activity conferred by vitamin $D$ and melatonin, beneficial effects of live childhood vaccines and microbiota. However, none of the mechanisms have been conclusively explained. Although several mechanisms have been proposed, further studies are needed to explore age-related differences in immune system function and host response to COVID -19. Once elucidated, the differences can perhaps help mitigate morbidity and mortality caused by COVID -19 . 


\title{
POSTER PRESENTATION
}

\section{ID127 PP091}

\section{Ocular Surface Squamous Neoplasia: A Snowy Complication}

\author{
Hassan, Faisal ${ }^{1}$, Yaakub, Azhany $^{1}$, Zakariah, Sakinah², Saizaul, Zubaidah ${ }^{4}$ \\ ${ }^{1}$ Department of Ophthalmology and Visual Science, School of Medical Sciences, Universiti Sains Malaysia, \\ 16150 Kota Bharu, Kelantan, Malaysia \\ 2Department of Ophthalmology, Hospital Raja Perempuan Zainab II, 15200, Kota Bharu, Kelantan, Malaysia \\ ${ }^{3}$ Department of Pathology, Hospital Raja Perempuan Zainab II, 15200, Kota Bharu, Kelantan, Malaysia \\ ${ }^{4}$ Department of Orthopaedics, Hospital Kuala Lumpur, Kuala Lumpur, Malaysia
}

86-year-old female with underlying hyperlipidemia on oral medication, presented with painless, progressive whitish growth over her left eye for 3 months duration. She was not known to have any other medical illnesses. The whitish growth was progressively increasing in size for 3 months, till it covered the lower $1 / 3$ of the cornea. Also noted a fleshy mass with feeding vessels at the temporal side of the conjunctiva. Denies any significant trauma, chemical injury, sudden loss of weight or appetite and her vision has been poor for many years. Vision remained at $6 / 18$, no improvement with pinhole, due to extensive chorio-retinal scarring. She underwent cataract surgery more than 10 years ago, not complicated with posterior chamber intraocular lens implantation. Her right eye had normal anterior segment findings, also had poor vision, limited to hand movement. Noted to be aphakic, but denies trauma, surgery or douching done. Differential diagnosis was conjunctival intraepithelial neoplasia (CIN), mucoepidermoid carcinoma, actinic keratosis, bitot spots, benign intrepithelial dyskeratosis, malignant melanoma. Total excisional biopsy was done under local anesthesia, including $3 \mathrm{~mm}$ normal conjunctival margin, then the area was cauterized. Sample was sent for histopathological examination. Histopathology confirmed diagnosis of squamous cell carcinoma by presence of conjunctival tissue lined by stratified squamous epithelium and hyperkeratosis. She was then started on Guttae mitomycin-C (MMC) $0.02 \%$ every 8 hours, to complete 6 cycles. Each cycle requires 1 week of topical MMC and 1 week hiatus before the next cycle. Malignancy should be suspected in elderly with conjunctival growth. Excisional biopsy should be considered for definite diagnosis and treatment. 


\title{
POSTER PRESENTATION
}

\section{ID128 PP092}

\section{The Effect of Fetal Movement Counting on Maternal Anxiety and Experiences in a Private Hospital}

\author{
Ahmad, Aini ${ }^{1} \operatorname{Lim} Y, Y^{1}$ \\ ${ }^{1}$ School of Nursing, KPJ Healthcare University College Nilai Negeri Sembilan
}

Fetal movement counting improves maternal self-screening for decreased fetal movement, and thereby contributes to prevent adverse pregnancy outcomes. However, fetal movement counting may also contribute to maternal anxiety. The specific study objective is to assess the effect of fetal movement counting on maternal anxiety and maternal experiences during antenatal period in a private hospital. We employed a quantitative quasi-experimental design, using a purposive sampling. Total of 73 pregnant women with $\geq 28$ weeks' gestation were recruited and allocated either to an intervention group that is to perform daily fetal movement counting $(n=40)$ and sharing the experience with a fetal movement counting chart and given the educational program. The control group, the participants are to follow the standard antenatal care $(n=33)$. The primary outcome was maternal anxiety, measured by Spielberger State-Trait Anxiety Inventory (STAl). Statistical analysis was performed using SPSS Version 22.0. A significant difference was found in both the mean Spielberger STAI-State and STAI-Trait anxiety score at post-test. The mean and SDs on Spielberger STAI-State anxiety scores at post-test were $46.78(S D=5.23)$ and $52.19(S D=8.27)$ for the intervention and control groups, respectively, a mean difference between the groups of $5.41(P<0.00)$. Moreover, the mean and SDs on Spielberger STAI-Trait anxiety scores at post-test were $46.33(\mathrm{SD}=6.11)$ and 51.12 $(S D=8.00)$ for the intervention and control groups, respectively, a mean difference between the groups of $4.79(\mathrm{P}<0.01)$. Eighty percent of the pregnant women responded favorably to the use of the fetal movement counting charts. Pregnant women who performed fetal movement counting intervention reported to experience less anxiety compared with those in the control group. Majority of the pregnant women in the intervention group considered the use of a fetal movement counting chart to be positive. 


\section{POSTER PRESENTATION}

\section{ID129 PP093}

\section{Audiology Students' Attitude Towards Online Simulated Clinical Training}

Quar, Tian Kar'; Ishak, Wan Syafira ${ }^{2}$; Yusoff, Nur Izzati ${ }^{1}$; Yusoff, Wan Nur Hanim¹; Abdul Aziz, Nadiah ${ }^{1}$; Jamaludin, Mohd Khairil Azahar ${ }^{1}$.

${ }^{1}$ Program Audiology, Centre for Rehabilitation \& Special Needs Study, Faculty of Health Sciences, Universiti Kebangsaan Malaysia, Kuala Lumpur, Malaysia

${ }^{2}$ Program Audiology, Centre for Healthy Ageing and Wellness, Faculty of Health Sciences, Universiti Kebangsaan Malaysia, Kuala Lumpur, Malaysia

The COVID-19 pandemic has ushered in a new era that requires radical thinking of redesigning educational institutions, teaching and assessment method. Although online teaching can be applied to most courses, the prohibition of face-to-face delivery has caused a serious problem to the clinical practicum. The movement control restrictions have prompted us to deliver our clinical teaching remotely using simulated patients. The study aims to measure the students' attitude towards the newly developed online simulated clinical training module. An online simulated clinical training module was developed based on multiple adult cases seen in our clinic. The clinical cases were simulated to allow students to practice their skills on history taking, hearing assessment, rehabilitation, feedback management and counselling. The clinical training was conducted using the Microsoft Teams video conferencing system and hearing assessment was done using an online simulator. After the training, the students completed a questionnaire to assess the effectiveness of the online training. All final year Audiology students $(n=17)$ participated in this study. Most of the students (88.2\%) agreed that online training is a good way of learning clinical skills. Most of them $(76.5 \%)$ also agreed that the cases cover important topics. However, only $58.9 \%$ of the students agreed that the online clinical training can replace the traditional training. Meanwhile, the other $35.3 \%$ were indecisive and $5.9 \%$ disagreed with the statement. Online clinical training has its advantages and can be considered as part of the teaching curriculum in future, at least for adult patients. Due to the wide range of behaviour and challenges in handling paediatric cases in the clinic, delivering online teaching and assessment targeting this population is yet to be validated. 


\section{POSTER PRESENTATION}

\section{ID130 PP094}

\section{COVID-19 Mortality, can it be Predicted?}

Mohamad Afiq Amsyar ${ }^{1}$, Gunenthira Rao ${ }^{1}$, Rozaini Mat Shah ${ }^{1}$

${ }^{1}$ State Health Department of Pulau Pinang

Coronavirus Disease 2019 (COVID-19) is a pandemic that has caused significant increases in morbidity and mortality. Understanding the risk of COVID-19 mortality will help in prioritizing prevention measures to the right population. The aim of this study is to identify the risk factors of COVID-19 mortality in Pulau Pinang, Malaysia. A case control study was conducted based on retrospective review of COVID-19 cases during the study period (1st January 2021 till 30th June 2021). Cases were divided based on their disease outcome: "death" and "discharged". 152 "death" cases were categorized as case group and 760 "discharged" cases were randomly selected as a control group forming a ratio of 1:5 between death (cases) and discharged (control) group. Multivariable logistic regression was performed to explore the risk factors for COVID-19 mortality. Over the study period, 30219 COVID-19 have been reported in Penang. 152 death cases were recorded over the same period making the case fatality rate (CFR) of $0.5 \%$. Majority of the death cases were elderly ( $>60$ years old) (70.3\%), male (70.4\%), Malaysian (98\%), unemployed $(71.7 \%)$, sporadic cases $(63.8 \%)$, and cases with comorbidities (82.2\%). Multivariate analysis revealed age group more than 60 years old aOR:2.80 $(95 \% \mathrm{Cl} 1.37,5.73)$, male gender aOR:2.91 $(95 \% \mathrm{Cl}$ 1.63,5.21), Malaysian nationality aOR:7.64 $(95 \% \mathrm{Cl} 2.14,27.32)$, unemployed aOR:2.46 $(95 \% \mathrm{Cl} 1.31,4.61)$ and presence of multiple comorbidities aOR:20.26 $(95 \%$ $\mathrm{Cl} 9.79,41.92$ ) were the significant factors for COVID-19 mortality in Pulau Pinang. In conclusion, age more than 60, male, Malaysian, unemployed and having comorbidities were significant risk factors of COVID-19 mortality. Focusing on these significant risk factors during outbreak investigation can help in preventing high CFR due to COVID-19. 


\section{POSTER PRESENTATION}

\section{ID131 PP095}

\section{Clinical Virtual Innovative Teaching (CVIT) in Undergraduate Surgical Students: A Pilot Study}

Jailani, Ruhi Fadzlyana ${ }^{1}$

${ }^{1}$ Faculty of Medicine and Health Sciences, Universiti Sains Islam Malaysia, Nilai, Negeri Sembilan, Malaysia.

COVID-19 has changed medical education delivery around the world due to suspension of clinical attachment and on-campus activities. Clinical Virtual Innovative Teaching (CVIT) is a newly designed medical education teaching pedagogy; whereby, interactive form of teleconferencing is adopted. The patient, students and lecturer are all located at different places and connected via a virtual platform. In this virtual clinical session the lecturer acts as a moderator and it is divided into 6 main component tasks. 1) History taking - effective communication skills of the students are being assessed and feedback from the real patient to the students. 2) Physical examination - videos, pictures or demonstrations of the real patient examination findings are shared. 3) Differential diagnosis - interactive discussion among the students and lecturer. 4) Investigation - the real imaging and blood results are shared within the class. 5) Management - prerecorded video of the surgical intervention is broadcasted during the class. 6) Debriefing - case summary and feedback session from the lecturer and students. Interactive discussion happened throughout the session to ensure two-way communication. A real patient case discussion in CVIT with component tasks, have enabled the students to experience authentic patient interaction on a virtual platform. Students felt more engaged and correlated their past clinical experience with a real patient encounter. This technique is replicable and most effective to be done in a small group with not more than ten students per group. A recorded version of the session provides the opportunity for the students to revise as well as sharing their virtual clinical teaching experience with other colleagues. It is more superior than using a simulated patient as this session is not scripted and enable students' accessibility to hospital clinical teaching environments virtually. 


\title{
POSTER PRESENTATION
}

\section{ID132 PP096}

\section{Rare presentation of spontaneous tonsillar hematoma in patient with underlying Immune Thrombocytopenia (ITP): a case report.}

Lye Meng Hon ${ }^{1,2}$, Intan Kartika Kamarudin ${ }^{3}$, Norhafiza Mat Lazim ${ }^{1}$

\author{
${ }^{1}$ Department of Otorhinolaryngology-Head and Neck Surgery, School of Medical Sciences, Universiti Sains \\ Malaysia, Health Campus, Kubang Kerian 16150 Kelantan, Malaysia. \\ ${ }^{2}$ Department of Otorhinolaryngology-Head and Neck Surgery, Hospital Sungai Buloh, Jalan Hospital, 47000 \\ Sungai Buloh, Selangor, Malaysia \\ ${ }^{3}$ Faculty of Medicine, Universiti Teknologi Mara, Selangor, Malaysia
}

Immune thrombocytopenia (ITP) is an acquired autoimmune disorder characterized by isolated thrombocytopenia without other discernable etiology. Reported intraoral manifestations included petechiae, purpura, ecchymosis, and hematoma at trauma prone site. Spontaneous tonsillar hematoma is rarely encounter. We present a case of spontaneous tonsillar hematoma in a patient with underlying ITP. A 15-year-old male with underlying ITP presented with fever and sore throat for two days with odynophagia, dysphagia, and change of voice. Examination revealed two large sanguineous mass occupying the oropharynx. Flexible endoscopy showed bilateral enlarged tonsils. Patient developed spontaneous bilateral epistaxis requiring nasal packing. Blood test showed elevated leucocytes count and thrombocytopenia. Contrast-enhanced (CT) scan revealed adenoid and palatine tonsils hypertrophy with bilateral cervical lymphadenopathy causing oropharyngeal airway narrowing. He was admitted for observation and started on intravenous antibiotics, corticosteroid and co-managed with medical team. Nasal packing was removed after 4 days following platelet transfusion. Patient was discharged after 7 days with full resolution of symptoms. The goal of treatment for ITP is achieving a sustainable hemostatic platelet count with minimal adverse drug effects. Acute treatment of major bleeds includes intravenous immunoglobulin, corticosteroids, and platelet transfusions. Platelet count is a poor indicator of bleeding. Close observation is paramount. Spontaneous hematoma of the tonsils without precipitating trauma is rarely encountered. CT-scan is indicated to delineate site and source of hematoma as well as eliminating the presence of vascular mass. The treatment of oropharyngeal hematoma depends on location, size, and compression on adjacent structure. Large tonsillar hematoma can produce ball-valve effect, leading to airway obstruction. Patient with symptomatic ITP should be co-managed with hematologist to ensure the best outcome. High index of suspicion should be practiced when encountering patient with erythematous oropharyngeal mass. Bleeding disorder should be excluded. Symptomatic ITP should be managed with multidisciplinary team approach. 


\section{POSTER PRESENTATION}

\section{ID140 PP097}

\section{A Review of Night Shift Work, as a Risk for Breast Cancer in Women}

Rozi, Ramiza Iylia'; Yusoff, Siti Nur Ainina Mohd'; Syed Mohideen, Fathima Begum² ; Yahaya, Yafizah²; Ganason, Anu Suria ; Mohd Noor, Muhammad Tauffik ${ }^{2}$

${ }^{1}$ Faculty of Medicine and Health Sciences, Universiti Sains Islam Malaysia, Bandar Baru Nilai,71800 Negeri Sembilan, Malaysia

${ }^{2}$ Family Medicine Unit, Faculty of Medicine and Health Sciences, Universiti Sains Islam Malaysia, Bandar Baru Nilai,71800 Negeri Sembilan, Malaysia

The demand for labour has increased worldwide due to extensive urbanization. In this modern society, there is an increasing prevalence of night shift work in various industries due to the rapidly growing demand for productivity across the time zone. Many companies offer shift work so that employees can work under the best conditions and companies can operate continuously. There is a high prevalence of women working nowadays. Scientists found that women who work night shifts for many years are more likely to develop breast cancer than other women. The main objective is to examine the interrelated cancer-promoting mechanism because of night shift work in women by reviewing other studies. The specific objective focuses on the effects of sleep deprivation in shift workers, which can contribute to breast cancer, and light exposure, which can lead to melatonin suppression, possibly carcinogenic. All information were retrieved from electronic databases such as PubMed, ScienceDirect.com, Google Scholar, Medline, and EBSCOhost. The keywords used were "shift work", "night shift work", and "breast cancer". Twelve studies were reviewed. The review showed, there was an association between the risk of breast cancer and circadian rhythm disorders on night shift workers. Circadian rhythm disorders cause decreased production of the tumour suppressor genes PER2. Whereas exposure to light at night, results in decreased melatonin production. Melatonin has anti-cancer properties. Therefore, statistically, a significant association between night shift work and breast cancer risk was present [OR (95\% $\mathrm{Cl})$ : 1.34 (1.05-1.72)]. Overall epidemiological evidence shows that female night shift workers had an increased incidence of breast cancer. However, the evidence is not entirely conclusive. Thus, more research is needed to explore and further focus on the existing epidemiological evidence. 


\section{POSTER PRESENTATION}

\section{ID142 PP099}

\section{Perineural Injection Therapy in Foot Drop: A New Hope?}

Ismail, Anis Afikah ${ }^{1}$; Hanafi, Muhammad Hafiz'1,2; Kassim, Nur Karyatee ${ }^{3}$; Ibrahim, Al Hafiz ${ }^{1,2}$

${ }^{1}$ Rehabilitation Medicine Unit, Hospital Universiti Sains Malaysia, Health Campus, Universiti Sains Malaysia, Kubang Kerian, 16150 Kota Bharu, Kelantan, Malaysia.

${ }^{2}$ Rehabilitation Medicine Unit, School of Medical Sciences, Health Campus, Universiti Sains Malaysia, Kubang Kerian, 16150 Kota Bharu, Kelantan, Malaysia.

${ }^{3}$ Basic Sciences and Oral Biology Unit, School of Dental Sciences, Health Campus, Universiti Sains Malaysia, Kubang Kerian, 16150 Kota Bharu, Kelantan, Malaysia.

Nerve inflammation is a mystery in the neuroscience community. Perineural Injection Therapy (PIT) is one of the emerging interventions that intrigue most clinicians in both its efficacy and safety. We presented an interesting positive finding of PIT in a foot drop patient following a 1-year traumatic injury. A 42-year-old gentleman presented to our clinic with complaint of left sided foot drop and neuropathic pain. His neurological and nerve conduction study supported the diagnosis of lower motor neuron injury of his left lower limb peripheral nerves. After proper discussion and consent, the patient received a weekly PIT for 8 weeks. PITs were administered along the distribution of the superficial peroneal nerve, deep peroneal nerve, tibia nerve, and sural nerve. Each injection consists of $3 \mathrm{~mL}$ solution (2.8mL of sodium bicarbonate in $100 \mathrm{~mL}$ of $5 \%$ dextrose solution), administered subcutaneously. Nerve conduction study, pain, and neurological assessment were determined before PIT and at the end of 8 weeks. The success of the treatment was determined by the decrease in subjective pain, increase muscle power, and nerve conduction study. The pain score improved from $8 / 10$ to $2 / 10$ at the end of the treatment. The Medical Research Council (MRC) muscle power scale also demonstrated a marked increment of muscle power which left ankle plantarflexion and toes flexion from $1 / 5$ to $4 / 5$ after completed 8 series of injections. The most fascinating and noteworthy progress was his nerve conduction study of left sural nerve showed a significant sign of recovery from absent amplitude before treatment, to $3 \mu \mathrm{V}$ value after 8 weeks of PITs. PIT is a cost-effective alternative in patient with nerve injury. Further research is warranted to understand the pathophysiology of the appealing phenomenon. 


\section{POSTER PRESENTATION}

\section{ID143 PP100}

\section{Development and Initial Validation of The Questionnaire Assessing the Perception and Practice of Workplace Violence Prevention At Healthcare Facilities}

\section{Mohd Nizam Mohamad Yazid ${ }^{1}$; Nik Rosmawati Nik Husain ${ }^{1}$; Aziah Daud ${ }^{1}$}

${ }^{1}$ Department of Community Medicine, School of Medical Science, University Sains Malaysia, Kelantan, Malaysia

A newly developed questionnaire is designed to assess the prevention of workplace violence. The usability of this questionnaire is to assess the perception and practice of workplace violence prevention among employers at healthcare facilities. The aim of this study is to establish content and face validity of a newly developed questionnaire assessing perception score and practice score of workplace violence prevention among employers at healthcare facilities. Existing literature were reviewed to establish the domains, develop and refine the items. There were 2 domains which perception with 59 items and practice with 41 items. Its content validity was measured by item-level content validity index(I-CVI) and scale-level content validity index $(\mathrm{S}-\mathrm{CVI})$ method. The content of the questionnaire was validated by 9 expert panels in the field. The efficacy of the questionnaire was further probed by testing the face validity of 10 employers in healthcare facilities using item-level face validity index(I-FVI) and scale-level face validity index(S-FVI) method. The content validity index for perception and practice of workplace violence prevention were determined to be 0.98 and 0.97 respectively. These indicate good relevancy of the 59 items used to assess the perception and 41 items used to assess the practice. Calculation of the face validity index resulted in value of 0.94 for perception and 0.95 for practice, therefore indicating that the questionnaire was easily understood by the employers. The newly developed questionnaire to assess the perception and practice of workplace violence prevention among employers at healthcare facilities was valid for its content and face validity indices. 


\title{
POSTER PRESENTATION
}

\section{ID149 PP102}

\section{Estimated Medical Costs of the Top-five Cardiovascular Cases in 2020 at one the University Hospitals in Malaysia}

\author{
Mohamad Yasim, Mohamad Helmi ${ }^{1}$; Shah Nizamuddin, Muhammad Khairul Asraf ${ }^{1,2}$; Abu Hasan, Nurhasnira; \\ Azzeri, Amirah ${ }^{1,3}$; Zainal Abidin, Imran ${ }^{4}$; Dahlui, Maznah ${ }^{1,2}$ \\ ${ }^{1}$ Department of Research, Development, and Innovation, University Malaya Medical Centre, Kuala Lumpur, \\ Malaysia \\ ${ }^{2}$ Department of Social and Preventive Medicine, Faculty of Medicine, University of Malaya, Kuala Lumpur, \\ Malaysia \\ ${ }^{3}$ Faculty of Medicine and Health Science, University Sains Islam Malaysia, Nilai, Negeri Sembilan, Malaysia \\ ${ }^{4}$ Cardiology Unit, Department of Medicine, University Malaya Medical Centre, Kuala Lumpur, Malaysia
}

Cardiovascular cases are commonly seen at University Malaya Medical Centre (UMMC). The diseases used up a considerable amount of hospital resources and incur significant costs. This study aims to establish the five most common cardiovascular diseases (CVD) according to the International Classification of Diseases, Tenth Revision (ICD-10) at UMMC and the associated annual costs of treatment. A retrospective review from the hospital information system was performed and the 'I' code was identified. The code was used because it reflects CVD codes in the ICD10. The final data were sorted according to the top-5 based on hospital statistics. Costs for the top- 5 cases were estimated based on data from one of the university hospitals in Malaysia (the price year 2020), which was calculated through top-down and activity-based costing approaches. The annual costs for each code were estimated based on the UMMC statistics. There were 72,520 cases with ' $I$ ' code in 2020 found from the hosiptal database. Of that number, the top-five codes were I10 (Essential (primary) Hypertension) [n=26323, 36.3\%] followed by 125.9 (Chronic Ischaemic heart Disease, unspecified) [n=5027, 6.93\%], 124.9 (Acute Ischaemic Heart Disease, unspecified) [n=4442, 6.13\%], 163.9 (Cerebral Infarction, unspecified) [ $\mathrm{n}=4188,5.77 \%$ ] and 150.0 (Congestive Heart Failure) [n=3621, 4.99\%]. The estimated costs for the 110, 125.9, 124.9, 163.0 and 150.0 were RM1,699.27, RM1,524.42, RM1,715.39, RM1,933.51 and RM1,697.69 respectively per treatment episode. The annual costs for the 110, 125.9, 124.9, 163.0 and 150.0 were RM44,729,884.21, RM7,663,259.34 RM7,619,762.38, RM8,097,539.88 and RM6,147,335.49 accordingly. The cumulative hospital costs from these five cases were approximately RM75 millions per year, which was $12.3 \%$ of the total hospital expenditure. The current findings revealed that CVS cases incurred significantly high costs in UMMC. This study allows UMMC to plan strategies and advocacies to minimise the clinical and economic burden of the diseases. 


\section{POSTER PRESENTATION}

\section{ID150 PP103}

\section{Instructors' Satisfaction towards Online Distant Learning for Clinical Nutrition Practice Courses}

Mazuin Kamarul Zaman ${ }^{1}$; Syahrul Bariah Abdul Hamid'; Ahmad lqbal Azam²

${ }^{1}$ Centre of Dietetics Studies, Faculty of Health Sciences, UiTM Cawangan Selangor, Kampus Puncak Alam, 42200 Kuala Selangor, Selangor, Malaysia

${ }^{2}$ Faculty of Business Management, UiTM Cawangan Selangor, Kampus Puncak Alam, 42200 Kuala Selangor, Selangor, Malaysia

COVID -19 pandemic challenges faculty to innovate delivery mode of clinical practice courses to meet its objectives despite not being able to participate in on-site training. This leads to additional preparatory tasks for instructors to design and conduct courses interactively despite the limitations. This study aims to assess the satisfaction of instructors for online distance learning (ODL) conducted for clinical nutrition practice courses. A cross-sectional study was conducted on clinical instructors of Centre of Dietetics Studies, UiTM, who supervises students for clinical training. Questionnaires on satisfaction towards ODL for clinical nutrition practice courses were distributed electronically. The questionnaire was based on the Online Instructor Satisfaction Questionnaire (OISQ). The previously validated, five-point Likert scale questionnaire consisted of 28 items, categorized into three sections: student, instructor, and institution. Eleven responses from all instructors for clinical nutrition practice courses were received (response rate of $100.0 \%$ ). Overall, only $18.2 \%$ of instructors were more satisfied with online teaching than the face-to-face method. Based on the survey, most instructors were satisfied with students during ODL: active participation (90.9\%) and flexibility (100\%) despite the less enthusiasm in online learning than traditional learning $(72.7 \%)$. Most instructors felt that it was a challenge to motivate students in online learning compared to traditional teaching $(72.7 \%)$. In the next section, the main challenge was addressed by instructors where more resources were used in online teaching compared to traditional teaching (63.6\%) and technical difficulties (45.5\%). The area of most dissatisfaction reported by instructors (in the institution section) was longer preparation time (54.5.\%). Instructors were dissatisfied with online distance learning for clinical nutrition practice courses despite positive perceptions towards students during ODL sessions and positive attitudes towards ODL. ODL for hands-on courses can be executed when physical limitations are in place. 


\title{
POSTER PRESENTATION
}

\section{ID151 PP104}

\section{Cybersecurity Issues of Medical Devices: Addressing the Threat and Suggestion to the Solution}

\author{
Amin, Nurul Syafiqah ${ }^{1}$; Mohd Saiful Sega, Intan Ku Khalilah ${ }^{1}$; Tajudin, Najiah ${ }^{1}$; Jamal Abdul Nasir, Nurul \\ Aisyah $^{2}$ \\ ${ }^{1}$ Faculty of Medicine and Health Science, Universiti Sains Islam Malaysia (USIM), Persiaran Ilmu, Putra Nilai, \\ 71800, Nilai, Negeri Sembilan, Malaysia \\ ${ }^{2}$ Faculty of Information Science and Technology, Multimedia University (MMU), Jalan Ayer Keroh Lama, 75450, \\ Bukit Beruang, Melaka
}

Implantable medical devices (IMDs) and software as medical devices (SaMD) are promising technology in medical field. As IT networks become more integrated into medical devices, cybersecurity is inevitably a concern. We describe the cybersecurity issues, including threats and suggestions. A literature search was conducted using databases such as Google Scholar, PubMed, and ScienceDirect. Relevant keywords included 'cybersecurity', 'healthcare cyber-attack', 'medical device cybersecurity' and 'remote monitoring' The above search yielded 60 articles, and we selected 15 based on their title, abstract, and full text. Cybersecurity vulnerabilities are weaknesses in a system that are critical to medical device functionality and that can be exploited. Cyber threats are increasing as wireless connectivity and internet access open attack opportunities. Lack of embedded device security control, as of the absence of cryptography becomes the greatest concern. Such assault may target the core system of medical devices. For example, the attackers may issue malicious programming commands to the system and interfere with its operationality. Likewise, applications that store and convey patient data may be accessed unauthorizedly, compromising confidentiality, resulting in disruption of medical infrastructure. The perpetrators can alter critical data that may falsify its reporting and cause failure of treatment. Mitigating the threat is, therefore, a shared responsibility. Manufacturers need to adopt international standards such as ISO/IEC 80001 and fulfil the regulation. For effective reporting of any issues, good feedback systems are needed between the manufacturers and healthcare providers. Cyber-hygiene by end-users is crucial. Patient should ensure that they are regularly installing updates and patches so that security standards are at the utmost level. Attention should chiefly be bought to these issues because of their detrimental effects on patient health and safety. Therefore, patients and clinicians should appreciate these risks alongside the convenience of the technology. 


\section{POSTER PRESENTATION}

\section{ID156 PP105}

\section{Performing the Proper Nasopharyngeal Swab in the COVID-19 pandemic}

$\underline{\text { Aman Shah, Normaisarah }}^{1}$; Salahuddin, Nor Azirah ${ }^{1}$; Md Arepen, Siti Asmat ${ }^{1}$; Hassan, Nor Eyzawiah ${ }^{1}$

${ }^{1}$ Faculty of Medicine and Health Sciences, Universiti Sains Islam Malaysia (USIM), Persiaran Ilmu, Putra Nilai, 71800. Nilai, Negeri Sembilan, Malaysia

Nasopharyngeal swab is an important procedure in diagnosing COVID-19. Healthcare worker must have enough exposure to this procedure to avoid false-negative result and complications. This study aimed to describe the anatomy involved in nasopharyngeal swab technique, to determine the correct technique of nasopharyngeal swab test for COVID-19 specimen collection, to evaluate common challenges and mistakes done during the nasopharyngeal swab test and its implications, and to compare the sensitivity of other technique of nasal samplings and nasopharyngeal swab test in detecting SARS-CoV-2 RNA. PubMed, Scopus and EBSCO host databases were searched to identify all the research studies which focused on nasopharyngeal swab technique. The search found 2816 results, by using keywords COVID-19, SARS-CoV-2, nasopharyngeal, swabs and technique. Fifty (50) relevant papers were identified based on titles screened. Fifteen (15) original full papers were obtained and reviewed which fitting the inclusion criteria. The distance between the nares and nasopharynx is estimated $10 \mathrm{~mm}$ less than distance between nares and tragus externally. Strict heath care worker protection with complete personal protective equipment is compulsory. The proper technique of swab insertion is included proper explanation, direct the swab gently close to the floor of the nose to past few resistances in nasal cavity. Proper technique will reduce pain and mucosa injury. Insufficient viral load or inadequate sampling such as if the swab fails to reach nasopharyngeal wall can be resulted in false-negative of nasopharyngeal swabs. Most of the studies reported that nasopharyngeal swab test has the highest sensitivity compared to other nasal samplings. The understanding of the nasal cavities' anatomy and the proper technique is crucial to avoid unwanted complications and to minimize false negative results. Thus, the nasopharyngeal swab could be useful in screening larger populations as specimens can be collected more rapidly and efficiently. 


\title{
POSTER PRESENTATION
}

\section{ID158 PP106}

\section{Comparison of Refractive Outcomes using Applanation or Immersion techniques for intraocular lens (IOL)power calculation in cataract surgery}

\author{
S, Sharan'; YL, Lee ${ }^{2} ;$ A,Zalifa Zakiah'; M,Azlina ${ }^{4} ;, R$, Jamaliah $^{1}$ \\ ${ }^{1}$ Deparment of Ophthalmology, Hospital Kuala Lumpur, Kuala Lumpur,Malaysia \\ ${ }^{2}$ Centre for Clinical Trial, Institute for Clinical Research, Hospital Ampang, Selangor,Malaysia \\ ${ }^{3}$ Deparment of Ophthalmology, Hospital Ampang, Selangor, ,Malaysia \\ ${ }^{4}$ Faculty of Medicine and Health science Universiti Sains Islam Malaysia, Bandar Baru Nilai, Negeri Sembilan, \\ Malaysia
}

The National Eye Survey has identified cataract as the leading cause of blindness in Malaysia and it can be treated by cataract surgery. Accurate biometry is an essential for cataract surgery. It facilitates correct intraocular lens (IOL) power calculation to achieve good post-operative visual outcome. IOL calculation can be obtained either by applanation, immersion or optical biometry. This study aims to compare post-operative refractive outcome between 2 methods of A scan Biometry in Hospital Ampang. This is a retrospective study analyses from Cataract Surgery Registry (CSR) record for patient who underwent phacoemulsification from 1st July 2018 until 31st December 2019 by Ophthalmology department in Ampang Hospital. IOL power calculation was obtained from either Applanation or Immersion method. The main outcome is to measure the percentage of cases achieving a postoperative spherical equivalent \pm 1.0 diopter (D) of target spherical equivalent between 2 methods of $A$ scan. A total of 1876 phacoemulsification were performed from July 2018 until December of 2019. A total of 1412 patients were analysed from the registry. An overall of $87.6 \%$ cases achieved within $1.00 \mathrm{D}$ of target refraction. Out of this, $89.1 \%$ patients achieved target refraction within 1.0D in the immersion group which is higher compared to the applanation group with $86.2 \%$. However, this value is not statistically significant with $p$ value of $0.098(p>0.05)$. This study concluded that both immersion and Applanation A-scan provide reliable pre-operative measurements for cataract surgeries. Choice of biometric technique had therefore no influence on the target spherical equivalent and post-operative visual outcome. 


\section{POSTER PRESENTATION}

\section{ID160 PP107}

\section{COVID-19, Dengue Fever and Euglycaemic Diabetic Ketoacidosis: Triple Threat. A Coinfection, A Serological Cross-reaction, or A Coincidence?}

Tan, Wei Ming ${ }^{1}$; Solehan, Hana Maizuliana ${ }^{1}$; Samad, Sarah Zulaikha ${ }^{1}$; Zanirah, Fatimah ${ }^{1}$; Mohammad Kazmin, Nur Ezzaty'; Sia, Koon Ket ${ }^{2}$

${ }^{1}$ Department of Medicine, Faculty of Medicine and Health Sciences, Universiti Sains Islam Malaysia (USIM), Nilai, Negeri Sembilan, Malaysia

${ }^{2}$ Department of Medicine, Ampang Hospital, Selangor, Malaysia

The spread of coronavirus disease 2019 (COVID-19) pandemic caused by severe acute respiratory syndrome coronavirus 2 (SARS-CoV-2) to the dengue endemic countries including Malaysia, poses a substantial diagnostic challenge to the treating physicians due to the similarity in their clinical presentations and laboratory parameters. We report a case of 56-year-old Malay gentleman, with underlying hypertension, ischemic heart disease and diabetes mellitus on empaglifozin, a sodium-glucose co-transporter 2 (SLGT2) inhibitor, who was initially treated as dengue fever with euglycaemic diabetic ketoacidosis (EDKA). He was referred from a private clinic with negative dengue NS1 antigen and positive IgM and IgG antibodies. He presented to us with 4 days of fever, cough, and vomiting. He was later confirmed to be positive for SARS-CoV-2 by reverse transcription-polymerase chain reaction (RT-PCR). Severe ketoacidosis (serum ketone $7 \mathrm{mmol} / \mathrm{L}$ and HCO3 8.2 $\mathrm{mmol} / \mathrm{L}$ ) likely resulted from SLGT2 inhibitor, swiftly resolved with intravenous hydration and intravenous infusion of insulin. Blood transfusion was given in view of suspicion of occult bleeding from dengue fever. Subsequently, he recovered with a tapering dose of dexamethasone and was weaned off nasal prong oxygen. Albeit there were reported cases of COVID-19 and dengue coinfection, false-positive dengue serology has been found in COVID-19 patients and vice versa. Precaution must be exercised when interpreting the result of rapid serological test. Confirmatory dengue PCR test should have been done in this case, but it is often time-consuming and provides no added therapeutic advantage in the patient management. Concurrent EDKA, an uncommon diabetic complication, adds to the challenge. Large-scale study on the cross-reactivity between dengue virus and SARSCoV-2 as well as the coinfection is imperative especially in Southeast Asia as it helps in the understanding and the accurate diagnosis of the two different disease entities with similar clinical manifestations. 


\title{
POSTER PRESENTATION
}

\section{ID161 PP108}

\section{A Cases Series of Endogenous Endophthalmitis in Poorly Controlled Diabetes Mellitus}

\author{
A. Sasikala ; AK. Adlina ${ }^{1}$; ZA.Zairah ${ }^{1}$; A,Zalifa Zakiah ${ }^{1} ;$ M,Azlina ${ }^{2}$ \\ ${ }^{1}$ Department of Ophthalmology Hospital Ampang, Selangor, Malaysia \\ ${ }^{2}$ Faculty of Medicine and Health Science, Universiti Sains Islam Malaysia, Bandar Baru Nilai Negeri Sembilan, \\ Malaysia
}

Endogenous endophthalmitis is an intraocular infection resulting from hematogenous bacterial spread in the body. It is relatively rare and is associated with immunocompromised states and debilitating diseases. Case 1: A 62-year-old female presented with fever and left knee swelling. She was diagnosed to have Disseminated Klebsiella pneumoniae bacteraemia with septic arthritis. Left eye examination suggestive of endophthalmitis with vitreous tap yielded Klebsiella pneumoniae. Although infection controlled clinically with intravitreal and systemic antibiotics, her vision deteriorated to non-perception to light. Case 2: A 51-year-old female presented with bilateral poor vision, right eye pain and swelling for 2 days. Diagnosis of bilateral endogenous endophthalmitis was established with presence of RE hypopyon and vitreous loculation and left eye macula abscess, supported by positive yield of Escherichia coli from vitreous tap. Her ultrasound revealed presence of multifocal left renal abscesses. Despite treatment for Disseminated Escherichiae coli bacteraemia, her vision remained poor, and her right eye progressed to panophthalmitis. Case 3: A 57-year-old male presented with right eye redness, pain and poor vision for 1 week. Impression of endogenous endophthalmitis was made upon positive culture of Klebsiella pneumoniae from his blood, urine and vitreous sample. Ultrasound finding showed presence of liver abscess. He was treated as Invasive Klebsiella Syndrome. Later, the eye progressed to pan-ophthalmitis and globe perforation requiring evisceration. Diabetes Mellitus is the most common risk factor for Endogenous Endophthalmitis. A high clinical suspicion is needed for early diagnosis and treatment. It is a sight threatening intraocular infection, and the visual outcome is unfavorable in poorly controlled diabetes patient. Recognition of primary foci is important in managing the spread of infection. Close monitoring of immunecompromised patients with systemic infections may enable early diagnosis and treatment and improve prognosis. 


\title{
POSTER PRESENTATION
}

\section{ID162 PP109}

\section{Nephroprotective Effects of Eleutherine americana Merr Against Lead Acetate-Induced Cytotoxicity in Mice BALB/C}

\author{
Jayanti, Neti Eka ${ }^{1,2}$; Mohd Said, Rozzana ${ }^{2}$; I'tishom, Reny ${ }^{3}$; Utami, Rinda Aulia ${ }^{4}$; Sumiati, Raudah, Siti'; \\ Achmad, Arisandy ${ }^{1}$; Jaiddin, Kasim Nurhas ${ }^{1}$; Sulfandi, Usman, Rezky Amaliah ${ }^{1}$ \\ ${ }^{1}$ Institute of Health Technology and Science Wiyata Husada Samarinda, East Kalimantan Indonesia \\ ${ }^{2}$ Department of Basic Sciences in Physiology, Universiti Tekhnologi MARA, Faculty of Health Sciences, Selangor \\ Malaysia \\ ${ }^{3}$ Department of Medical Biology, Faculty of Medicine, Universitas Airlangga, Surabaya, Indonesia \\ ${ }^{4}$ Faculty of Health Sciences and Nursing, Muhammadiyah Semarang University, Indonesia
}

Lead is a poisonous metal, hurtful to most human body organs if exposure surpasses a permissible level of $50 \mu \mathrm{g} / \mathrm{m} 3$ and an action level of $30 \mu \mathrm{g} / \mathrm{m} 3$. This occurs through the initiation of reactive oxygen species (ROS). Studies have indicated that Eleutherine americana Merr contains profound biological properties which protects against cancer cells, decreased in prothrombin level, and vessel vasoconstriction. It will be interesting to study the effect of the plant extract on kidneys exposed to lead acetate. This study aimed to evaluate the nephroprotective effects of Eleutherine americana Merr extract on lead acetate cytotoxicity in mice BALB/c. A total of $25 \mathrm{BALB} / \mathrm{c}$ mice were randomly divided into five groups. Group 1 was given $0.5 \% \mathrm{Na}-\mathrm{CMC}$ orally while Group 2 was treated orally with $0.075 \mathrm{~g} / \mathrm{kg}$ body weight lead acetate $(\mathrm{Pb}(\mathrm{CH} 3 \mathrm{COO}) 2)$. Group 3 to 5 was given different dosages of Eleutherine americana Merr of 30,60 and $120 \mathrm{mg} / \mathrm{kg}$ body weight accordingly and simultaneously with $\mathrm{Pb}(\mathrm{CH} 3 \mathrm{COO}) 2$. All were treated for 35 days. Mice were sacrificed after 35 days; blood samples were collected for analysis of creatinine and urea while the kidneys were for histological studies. The levels of creatinine and urea was significantly higher in $\mathrm{Pb}(\mathrm{CH} 3 \mathrm{COO}) 2$ treated mice $(\mathrm{p}<0.05)$. Treatment with the plant extract significantly reduced the level of blood creatinine and blood urea at extract concentration of $30 \mathrm{mg} / \mathrm{kg}$ body weight $(\mathrm{p}<0.05)$. Histology studies of the kidneys showed that $\mathrm{Pb}(\mathrm{CH} 3 \mathrm{COO}) 2$ caused glomeruli atrophy and tubular destructions. Treatment with the plant extract at dosages of 30 and $60 \mathrm{mg} / \mathrm{kg}$ body weight seemed to ameliorate the effect $\mathrm{Pb}(\mathrm{CH} 3 \mathrm{COO}) 2$ on the structure of the nephrons. Extract of Elutherine americana Merr was shown to have nephroprotective effect against the assault of $\mathrm{Pb}(\mathrm{CH} 3 \mathrm{COO}) 2$ in mice. 


\title{
POSTER PRESENTATION
}

\section{ID163 PP110}

\section{Severe Thrombocytopenia Following Glycoprotein Ilb/IIla Post PCI: To Wait or To Treat?}

\author{
Mohammad Kazmin, Nur Ezzaty ${ }^{1}$; W.J.Tiau ${ }^{2}$; Nordin, Fatimah Zanirah ${ }^{1}$; Solehan, Hana Maizuliana ${ }^{1}$; Tan, Wei \\ Ming $^{1}$ \\ ${ }^{1}$ Faculty of Medicine and Health Sciences, Universiti Sains Islam Malaysia (USIM), Nilai, Negeri Sembilan, \\ Malaysia \\ ${ }^{2}$ Cardiology Unit, Department of Medicine, Universiti Kebangsaan Malaysia Medical Centre (UKMMC)
}

Tirofiban induced thrombocytopenia is a rare cause $(<0.5 \%)$ of severe thrombocytopenia $(<50 \times 109 / \mathrm{L})$ in post percutaneous coronary intervention $(\mathrm{PCl})$. To date, no specific guideline is made available and most published cases have been managed actively with corticosteroid, intravenous immunoglobulins, or platelet transfusion. We report a successful conservative management of severe thrombocytopenia in a 62-year-old man post $\mathrm{PCl}$ for acute myocardial infarction. Full blood count $(\mathrm{FBC})$ on arrival showed hemoglobin $13.8 \mathrm{~g} / \mathrm{dL}$, white cell count $10.3 \times 109 / \mathrm{L}$, and platelet $158 \times 109 / \mathrm{L}$. PCl showed acute occlusion of proximal left anterior descending artery and he received tirofiban intracoronary infusion, followed by intravenous infusion. Repeated FBC twice, less than 24 hours showed a drop of platelet count to $20 \times 109 / \mathrm{L}$, confirmed by full blood picture. In less than 12 hours of withholding tirofiban and double antiplatelet, platelet count increased steadily, and he was resumed back on cardiprin on day 3 (platelet 51×109/L) and clopidogrel on day 5 (platelet $87 \times 109 / \mathrm{L}$ ) post procedure. Throughout, the patient had only minimal bleeding from gums, and his platelet normalized upon discharge. Platelet transfusion has been suggested once platelets $\leq 20 \times 109 / \mathrm{L}$ while Said et al recommends transfusion only when there is bleeding associated. In severe cases, intravenous immunoglobulins and corticosteroid have been used, although there is no data to specifically advocate this approach. In this case, despite the acute and severe nature of thrombocytopenia, withdrawal of tirofiban infusion alone was sufficient to bring platelet level back to baseline without any severe complication. Conservative management by stopping tirofiban and temporary withdrawal of antiplatelets are safe and should be considered as the preferred option even in case of severe tirofiban induced thrombocytopenia. Close observation versus active management to increase platelet count must be weighed judiciously against high thrombotic risk in patients with recent acute myocardial infarction. 


\title{
POSTER PRESENTATION
}

\section{ID164 PP111}

\section{Infective Endocarditis Secondary to Group B Streptococcus - Rare Manifestation of Invasive GBS Infection in Adult}

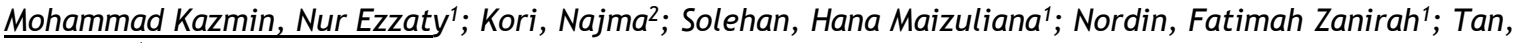 \\ Wei Ming ${ }^{1}$ \\ ${ }^{1}$ Faculty of Medicine and Health Sciences, Universiti Sains Islam Malaysia (USIM), Nilai, Negeri Sembilan, \\ Malaysia \\ ${ }^{2}$ Infectious Disease Unit, Department of Medicine, Universiti Kebangsaan Malaysia Medical Centre (UKMMC)
}

Group B Streptococcus (GBS) is a common organism causing infections among neonates as well as pregnant women. However, invasive infections among nonpregnant adults are rare, with infective endocarditis (IE) being one of the most severe presentations associated with $45 \%$ risk of mortality. We report a 52 -year-old man with hypertension and diabetes mellitus who presented with aortic valve insufficiency and GBS bacteremia. Echocardiogram revealed a $0.4 \times 0.7 \mathrm{~cm}$ vegetation at the right coronary cusp of the aortic valve. Despite six weeks of parenteral treatment with ceftriaxone, there was no decrement in the vegetation size. Multidisciplinary team discussion was held and after taking into consideration the high risk of embolism from large vegetation and due to severe aortic regurgitation, the patient was advised to undergo valve replacement surgery which he unfortunately refused. At six months' post therapy, the patient had multiple admissions for decompensated heart failure due to severe aortic valve incompetence although he does not have any clinical features to suggest systemic embolism to date. Despite successful measures taken to reduce morbidity related to neonatal GBS infection, the past two decades has witnessed a 4-fold increase of invasive GBS infection among adults with incidence ranging from 4.1 to 7.2 cases per 100,000 non-pregnant adults. IE caused by GBS infection among adults is uncommon and accounts for only a minority of all cases of bacterial endocarditis (1.7\%). It is associated with a high rate of metastatic disease and pulmonary embolism due to early development of large vegetations. Surgical treatment may improve outcomes of the patient. GBS is an uncommon but potentially life-threatening cause of IE in middle-aged adults with predisposing medical illnesses and physicians should be aware of the exceedingly high risk of complications and mortality rate associated with this disease. 


\title{
POSTER PRESENTATION
}

\section{ID166 PP113}

\section{Identification of Prognostic Significance of MDM2 in Nasopharyngeal Carcinoma using Integrative Bioinformatics Analysis}

\author{
Che Ismail Che Lah' ${ }^{1}$, Nik Yusnoraini Yusof ${ }^{2}$, Norhafiza Mat Lazim¹, Sarina Sulong ${ }^{3}$, Zilfalil Alwi ${ }^{3}$, Baharudin \\ Abdullah ${ }^{1}$ \\ ${ }^{1}$ Department of Otorhinolaryngology-Head \& Neck Surgery, School of Medical Sciences, Universiti Sains \\ Malaysia, Health Campus, 16150 Kubang Kerian, Kelantan, Malaysia. \\ ${ }^{2}$ Institute for Research in Molecular Medicine, Universiti Sains Malaysia, Health Campus, 16150 Kubang Kerian, \\ Kelantan Malaysia. \\ ${ }^{3}$ Human Genome Center, School of Medical Sciences, Universiti Sains Malaysia, Health Campus, 16150 Kubang \\ Kerian, Kelantan, Malaysia.
}

The role of mouse double minute 2 homolog (MDM2) gene in nasopharyngeal carcinoma (NPC) has been addressed comprehensively in many studies. However, the prognostic significance of MDM2 remains unclear in NPC patients. Understanding the interaction hub genes involved in NPC metastasis could lead to effective approaches to early detection of this cancer. To investigate the mRNA gene expression profile in the NPC samples using quantitative real-time PCR (qPCR) analysis and identify the hub genes underlying molecular mechanisms of NPC by bioinformatics approach. Quantitative real-time PCR was used to test the expression of MDM2, BCL2 and PIK3CA in clinical samples. Data from relevant analysis of qPCR were screened to explore the clinical role of MDM2 in NPC. Subsequently, MDM2related signaling pathways were investigated via bioinformatics approaches. An expression profile GSE646344 of the mRNA was extracted from the Gene Expression Omnibus (GEO) database. Annotation, Visualization and Integrated Discovery were applied to perform Gene Ontology (GO) and Kyoto Encyclopedia of Genes and Genomes (KEGG) pathway enrichment analysis. The protein-protein interaction (PPI) network of these DEGs was constructed using STRING software. MDM2 was expressed higher in the patient's sample compared with the normal individuals. The qPCR analysis in NPC specimens showed the positive expression ratio of MDM2 was 11.899, whereas expression of BCL2 and PIK3CA was lower at 6.518 and 1.852 respectively. A total of 30 genes ( 25 upregulated and 5 downregulated) were identified as hubs genes involved in NPC. PPI Network showed a strong interaction between MDM2, BCL2 and P53 protein but less association with PIK3CA. The high expression of MDM2 indicates that MDM2 may be an effective predictive indicator for NPC. We identified some key genes that systematically presented the biological processes and signaling pathways closely related to NPC initiation and development. 


\section{POSTER PRESENTATION}

\section{ID167 PP114}

\section{Promoting Ethical Use of social media Among Healthcare Professionals in the Digital Era}

Katiman, Helwa Husna ${ }^{1}$; Yunus, Aimi Mohd ${ }^{1}$; Muuti, Muhamad Zaid ${ }^{1}$

${ }^{1}$ Department of Medical Ethics and Law, Faculty of Medicine, Universiti Teknologi MARA, Sungai Buloh, Malaysia

Social media are undoubtedly one of the important tools for disseminating information, with a detrimental role in public health education. During this critical COVID-19 pandemic, prominent healthcare figures strategically use social media platforms, such as Twitter or Facebook, to keep people abreast of COVID-19 statistics and other relevant health concerns. Moreover, healthcare professionals use social media to remain in touch with colleagues while also reaching out and networking with the larger population. Concerns have arisen following the unethical use of social media among healthcare professionals. This study aimed to highlight the prevailing cases of healthcare professionals' unethical usage of social media. We adopt a doctrinal methodology by conducting an in-depth analysis of primary and secondary legal resources such as statutory materials, case laws, professional guidelines, and disciplinary hearing transcripts to determine the ethical standards expected from healthcare professionals. Additionally, commentary and argumentative analysis will be utilized to address the controversial social media posts by Malaysian healthcare professionals. Four cases of unethical use of social media have been analyzed that outlines four areas for critical rethinking, which include (1) maintaining confidentiality in social media; (2) maintaining the reputation of the medical profession; (3) maintaining patient-doctor boundaries; and (4) appropriate use of social media for public education. Overall, we conclude that social media platforms have considerable benefits in disseminating scientific insights and credible information for health promotion and education to combat the antivaccination movement. Nevertheless, a clear and proper set of rules and standards is needed to ensure that healthcare professionals' interactions with social media are guided to protect healthcare professionals and the public from potential harm while balancing the benefits of information sharing with privacy protection. Doctors particularly need to be cognizant of the relevant standards and maintain appropriate professional boundaries when using social media platforms. 


\title{
POSTER PRESENTATION
}

\section{ID169 PP115}

\section{The Uprising Homebirth Trend}

\author{
Yusoff, Nurul Afifah ${ }^{1}$; Masri, Maizatul Azma ${ }^{1}$ \\ ${ }^{1}$ Faculty of Medicine and Health Sciences, Universiti Sains Islam Malaysia, Nilai, Malaysia
}

The perinatal care services have improved over the years as evidence by the decline of maternal and neonatal mortality rates as most pregnant mothers were choosing hospital delivery. However, the homebirth trend still exists in Malaysia. There were cases of maternal and neonatal deaths that occur in Malaysia due to homebirths that were assisted by an untrained midwife or doula. We conducted a review on the homebirth practices looking into the prevalence, the reasons and also the safety of homebirths. Several platforms were used including PUBMED, UpToDate, Medline and Google scholar. Several articles, papers and journals from the internet had been reviewed based on the topic's importance. In Malaysia, increasing births attended by skilled personnel lead to the decreasing maternal mortality rate from 1990 until 2012. However, less than $2 \%$ of mothers practising homebirth or births that was attended by unskilled personnel. Homebirth was preferred due to various factors. The avoidance of any intervention is the commonest reason why they preferred homebirth rather than hospital delivery. Studies showed that $50 \%$ chose homebirth to avoid any intervention such as augmentation of labour, pain relief and caesarean section. 34.1\% chose comfort and privacy as the reason for having homebirth and others due to previous bad hospital experience, autonomy and to have natural birth. Homebirth by skilled staff among low-risk mothers are associated with lower complications. Homebirth complications include postpartum haemorrhage, retained placenta, asphyxia and early neonatal death. Accessibility to hospital obstetric unit also plays an important role to ensure good outcome. In Malaysia recently, 58 cases where unskilled births whereby 5 cases were homebirths attended by doula. Unfortunately, there were reported maternal and neonatal mortality from these unskilled homebirths. Homebirth specifically unskilled births increase maternal and neonatal risks compared to hospital delivery and therefore should be avoided. 


\section{POSTER PRESENTATION}

\section{ID170 PP116}

\section{Development and Utilisation of Breastfeeding Apps to Increase Breastfeeding Rates: A Scoping Review}

$\underline{\text { Mohammad Khuzaini, }} \underline{\text { Aliyyah }^{1}}$

${ }^{1}$ Medical-Based Specialties Department, Universiti Sains Islam Malaysia, Nilai, Malaysia

The World Health Organisation (WHO) Ten Steps to Successful Breastfeeding framework emphasizes the need for antenatal discussions regarding the importance of breastfeeding and postnatal practical support to initiate and troubleshoot breastfeeding issues. However, the COVID-19 pandemic has changed many facets of antenatal and postnatal care. Antenatal follow-ups are reduced in frequency and duration and mothers are discharged earlier after delivery. Furthermore, postnatal follow-ups are limited due to shortage of healthcare workers, infection control and apprehension to visit health care facilities during pandemic times. This further reduces the support received by mothers to initiate and continue breastfeeding. The aim of this review is to map the available evidence on breastfeeding mobile applications used to improve breastfeeding rates. A systematic literature search was undertaken on Pubmed, Cochrane Central Register of Controlled Trials and Scopus databases. The search was limited to primary studies carried out between the year 2000 and July 2021 describing development and usage of breastfeeding mobile applications to improve breastfeeding rates. Screening of titles and abstracts were performed prior to retrieval of full papers for further screening. 22 studies were included in the final analysis. From these included studies, 10 articles examined effects of the mobile application on breastfeeding, 5 studies focused on development of the application, 4 studies studied feasibility and usability and 4 studies examined both usability and effects on breastfeeding. There was no documented Malay language breastfeeding application studied in the literature. We propose the development of a breastfeeding mobile application in Malay as an adjunct to current strategies carried out to improve breastfeeding rates among mothers. 


\section{POSTER PRESENTATION}

\section{ID171 PP117}

\section{Students' Perspective on the Case-Based CPG Discussion Learning Method}

Zulkifli, Nursyazwani Aimi ${ }^{1}$; Omar, Mohd Rahman ${ }^{2}$

${ }^{1}$ Faculty of Medicine and Health Sciences, Universiti Sains Islam Malaysia, Nilai, Malaysia

${ }^{2}$ Department of Medicine Based Discipline, Faculty of Medicine and Health Sciences, Universiti Sains Islam Malaysia, Nilai, Malaysia

Ever since the emergence of COVID-19, students' placement in the clinical setting had been limited. Therefore, a case discussion based on the current Clinical Practice Guideline (CPG) discussion was introduced as a self-learning module to encourage students to enhance their critical thinking in managing cases bases on current CPGs. Students were divided into small groups, and cases were given beforehand. Discussions were conducted in 2 phases. First, in the groups without the lecturer's supervision, they were required to determine their learning objective themselves, evaluate the case thoroughly, come up with the differential diagnosis, case summary, investigations, management, prevention, and social, Islamic and figh/ethical and relate it to the appropriate CPG. Second, they also needed to reflect and remark on the investigations and management performed in the case. A discussion with the lecturer followed to confirm their overall comprehension of the topic and the CPG. From a student's perspective, students could pick up essential points when going through the CPG and memorize them more effectively as they could relate to the cases given. Students were also able to sharpen up their critical thinking skills when analyzing the case. Finally, under lecturer guidance, they better understand the topic and its management and emphasize essential points that may be missed. Reflection on the session enhances students' ability in self-directed learning, groups dynamic and cooperation, soft skills, and critical thinking. The method is very beneficial and effective in helping the students have a more profound knowledge of the principle of management and the disease itself. It enhances teambased discussion, self-directive initiative and is based on actual case-based discussion. 


\section{POSTER PRESENTATION}

\section{ID172 PP118}

\section{Correlation between Tobacco Smoking and Risk of COVID-19 Infection: A Literature Review}

Izuddin, Ummu Farhah' ${ }^{1}$; Abdul Muna'aim, Maimunah'

${ }^{1}$ Faculty of Medicine and Health Sciences, Universiti Sains Islam Malaysia, Nilai, Malaysia

Tobacco smoking is one of the major risk factors for mortality and morbidity of noncommunicable diseases. It is the most associated substance-use disorder that primarily targets the respiratory tract, like the pathologies seen in coronavirus disease 2019 (COVID-19), which is caused by severe acute respiratory syndrome coronavirus 2 (SARS-CoV-2). This review aims to study the relationship between tobacco smoking and the risk of COVID-19 infection, and to explore the influence of smoking status on COVID-19 infection severity and mechanism of smoking towards COVID-19 infection. Literature search was conducted based on relevant keywords on electronic databases such as PUBMED and Google Scholar. Several journals and papers were obtained based on topic's relevancy and reviewed. Ten studies were identified. Eight of the studies showed prevalence of smokers among COVID-19 patients was low with postulated mechanisms of nicotine protection characteristic in which it protects against hyperinflammation. The protective component from tobacco smoking were from nicotine, nitric oxide and angiotensin-converting enzyme-II (ACE-II). However, two studies considered the association between smoking history and development of severity of COVID-19 infection was 1.5 to 2 times more frequently found in past and current smokers. One study mentioned factors such as the act of smoking that increases the risk of virus transfer from hand to mouth, upregulation of ACE-II receptor which responsible for the viral entry site, virus-activated cytokine storm and the role of cellular protease and nicotinic acetylcholine receptor in viral entrance. At the time of this review, the available evidence suggests that smoking influences the severity of COVID-19 patients, although low prevalence of smokers among COVID-19 patient. Nevertheless, more well-designed prospective studies are needed to further validate the reported findings. 


\section{POSTER PRESENTATION}

\section{ID173 PP119}

\section{Phone Apps - Hope for Mental Health Management Amongst Students During Lockdowns}

Ismail, Suraya $;$; Abdullah Suhaimi, Afiqah ${ }^{1}$; Azmi, Liyana ${ }^{1}$

${ }^{1}$ Faculty of Medicine and Health Sciences, University of Islamic Science Malaysia, Negeri Sembilan, Malaysia

Anxiety, stress, and depression are the most common mental health disorders amongst students of all ages. The ongoing COVID-19 pandemic, combined with the implemented lockdowns have also exacerbated the rates and severity of mental health issues for students. Prior to COVID-19, students can seek treatment through physical counselling, de stress by exercising and socializing. However, the 'new norm' onset COVID-19 has imposed many limitations for mental health treatment particularly for students. An alternative for mental health treatment is through the usage of phone apps. This study aims to review examples and discuss the potential phone apps that can manage mental health issues, namely stress, anxiety, and depression amongst students during COVID-19 lockdowns. We used search strategy using the keywords 'anxiety disorder', 'stress', 'depression' and 'students. The literature review was done using Medline, PUBMED electronic databases, journals, and articles on relevant topics. We reviewed twelve studies which supports the use of phone apps in managing mental health issues amongst students. Phone apps like 'Calm' and 'Destressify' were able to reduce the stress, anxiety, and depression in students. Four studies showed that the continued use of phone apps enabled students to track their mental health condition over a period of 2 weeks - 6 months. However, these apps were only used as a mean for stress management and have yet to be used as the main treatment for stress amongst students. But future prospective for phone app usage for mental health management amongst students is bright due to the practicability, ease of use and safe due to less contact. Digital health apps could be used as alternative means of treatments to reduce anxiety disorder, stress, and depression among students during COVID-19 lockdown. Next step is to increase further studies to improve clinical evidence and future references. 


\title{
POSTER PRESENTATION
}

\section{ID174 PP120}

\section{Acute Symptomatic Focal Seizure with Todd's Paralysis as an Initial Presentation of Acute Stroke in A Non-Epilepsy Centre, A Case Report}

\author{
Solehan, Hana Maizuliana ${ }^{1}$; Tan, Wei Ming ${ }^{1}$; Zanirah, Fatimah, Mohd Kazmin, Nur Ezzaty ${ }^{1}$; Samad, Sarah \\ Zulaikha $^{1}$; Sharil, Nor Safiqah 1 ; A Bakar, Mohd Firdaus ${ }^{1}$; Norazinizah Ahmad Miswan ${ }^{2}$
}

${ }^{1}$ Faculty of Medicine and Health Sciences, Universiti Sains Islam Malaysia, Nilai, Malaysia ${ }^{2}$ Hospital Ampang, Kuala Lumpur, Malaysia

Todd's paralysis is a rare postictal condition in about $0.6-13.4 \%$ of patients with seizures and it is more common in focal seizure. The first onset of focal seizure without a history of focal neurological insult is a diagnostic challenge, especially in a non-neurology center. A 50-year-old lady with underlying diabetes mellitus, presented with multiple episodes of right arm tonic-clonic movement for a few seconds, followed by right arm weakness and dysarthria for a few hours. The event occurred multiple times per-day. The patient was conscious throughout each event. In between the episodes, she had normal muscle power. Her vital signs and other systemic examinations were normal. Blood investigations showed poor diabetic control (HbA1c-8.2\%). MRI brain revealed acute infarct at left basal ganglia. There was no electroencephalogram (EEG) facility in this hospital to confirm the seizure. Clinically, the patient was treated as a focal seizure. Intravenous phenytoin loading and maintenance dose were commenced, followed by oral levetiracetam. Antiplatelet, anti-lipid and oral hypoglycemic agents were also initiated. The right UL seizure and dysarthria reduced in frequency. EEG was arranged as an outpatient at another centre, but the patient defaulted. A follow-up review revealed a stable, controlled seizure with intermittent breakthrough episodes whenever she missed her medications. A seizure that occurs within one week of stroke onset is defined as an acute symptomatic seizure. Most post-stroke seizures is caused by hemorrhage or large vessel stroke and of cortical origin. However, this patient had ischemic lacunar. Todd's paralysis following the patient's focal seizure can be mistaken for hemiparetic limb caused by the stroke. Focal seizure can mimic myoclonus or dystonia, which may further confuse the clinicians. Hence, relying on clinical experience is crucial, especially when there is a lack of EEG facility in the clinical settings. 


\title{
POSTER PRESENTATION
}

\section{ID178 PP123}

\section{Estimating the Magnitude of Out-Of-Pocket Expenditure on Family Planning: Evidence from Malaysia}

\author{
Azzeri, Amirah; Jaafar, Hafiz; Mohamad Nor, Nadeeya 'Ayn; Abdullah, Amalia; Rosli, Asyiqin; Allani Huslan, \\ Asyiqin; Yazid, Nadia; Rahim, Asyraf; Zainoodin, Munir; Saidin, Humaira; Zulkifli, Nur Hanisah; Muji, \\ Nuraisya; Khair, Najmi; Ab Rahman, Amnan; Lukman, Saiful Syahmi; Basri, Taufeeq ; S.Bahri, A. Hisham ; Abol \\ Kasim, Balqis Wan Izudin, Wan Athirah; Mohd Zin, Noorsuwaidah; Mohd Noor, Aidil; Rushdi, Firdaus; Azahari, \\ Effa
}

Public Health Unit, Department of Primary Health Care, Faculty of Medicine and Health Sciences, Universiti Sains Islam Malaysia

Family Planning (FP) services are available in both public and private healthcare facilities in Malaysia. Several groups of people are eligible to receive free-of-charge services while some people are required to incur out-of-pocket (OOP) expenditure on FP. To date, local data on the magnitude of financial implications for FP is limited. This study was conducted to describe the monthly OOP expenditures incurred for FP and factors associated with the expenditure. A cross-sectional study was conducted among adults in Malaysia from April to June 2021. Data collection was conducted through an online survey via Google form. Information on sociodemographic profiles, socio-economic status, family planning utilisation and OOP expenditures on FP were collected. Descriptive and bivariate analyses were conducted accordingly. 473 people participated in the online survey, in which majority were female (83\%), Malay (98\%), Muslim (99\%), Married $(89 \%)$, has a university degree (58\%) and employed (84\%). The mean [SD] age of the study participants was 41.3[10.38]. The median [IQR] monthly household income was RM7000[6000]. 415(88\%) of them reported that the use of FP did not affect them financially while $358(75 \%)$ believed that the FP is not expensive. $244(52 \%)$ of the participants reported incurring a median [IQR] OOP expenditure of 100[150] monthly for FP. Older age and higher household income were significantly associated with the higher OOP expenditure for FP ( $p$-value <0.05). Otherwise, gender, race, religion, academic background, and employment status were not significantly associated with the OOP for FP. This study found that FP is highly affordable. Most of the participants reported that they did not incur any OOP expenditure and receive fully subsidized services in public healthcare facilities. This study highlights that the FP services provided by the government are beneficial to improve family well-being and prevent unwanted pregnancies without burdening the people financially. 


\title{
POSTER PRESENTATION
}

\section{ID182 PP126}

\section{Breastfeeding vs Artificial feeding - The Cognitive Development}

\author{
Zamri, Jannatunnajwa'; Masri, Maizatul Azma
}

${ }^{1}$ Faculty of Medicine \& Health Sciences, Universiti Sains Islam Malaysia, Nilai, Negeri Sembilan, Malaysia

Infant feeding either breastfeeding or formula feeding is differed by their contents that act as nutritional factor towards cognitive development. This review was to determine the association between method of feeding in infant either breastfeeding or formula feeding with their cognitive development. Literature review performed using electronic databases to search for relevant articles, journals, or textbooks. Globally, every child recorded $95 \%$ as ever breastfed in some points of their lives with variable practices on exclusive breastfeeding in different countries. In Malaysia, National Health and Morbidity Survey 2016 accounted 47.1\% as overall prevalence for exclusive breastfeeding below 6 months old infants which depend on several factors. This review showed there is an association between breastfeeding or formula feeding affecting on cognitive development. Beneficial effects among breastfeeding infant and to the mothers are observed. It is mainly due to the contents of breastmilk itself which include long chain polyunsaturated fatty acid (LCPUFA) such as arachidonic acid and docosahexaenoic that are linked to the neurodevelopmental effect on child as compared to formula-fed infant. Besides that, the two common neurodevelopmental disorders in Malaysia namely attention deficit hyperactivity disorder (ADHD) and autism spectrum disorder (ASD) highlighted the significant correlation respectively with infant's feeding method either breastfeeding or formula feeding which both balanced either giving positive or negative effect towards the disorder. Breastfeeding does give more advantageous effect towards cognitive development as compared to formula-feeding simultaneously prompt encouragement of exclusive breastfeeding among the mothers. 


\title{
POSTER PRESENTATION
}

\section{ID183 PP127}

\section{The Prevalence of Refractive Error Among Primary School in Gombak District, Selangor Prior To COVID-19 Pandemic.}

\author{
Abdul Muna'aim, Maimunah ${ }^{1}$; Zainol, Norlelawati ${ }^{1}$; Mokhtar, Azlina ${ }^{1}$; Khairudin, Muhammad Najmi ${ }^{1}$; Ghani, \\ Nor Diyana ${ }^{2}$ \\ ${ }^{1}$ Ophthalmology Unit, Department of Surgery, Faculty of Medicine and Health Sciences, Universiti Sains Islam \\ Malaysia, Negeri Sembilan, Malaysia \\ ${ }^{2}$ Our Optometrist, Klinik Pakar Kesihatan USIM, Negeri Sembilan, Malaysia
}

School children are considered a high-risk group for visual impairment. Uncorrected refractive errors and amblyopia can affect their learning abilities, physical and mental development. This study aims to determine the prevalence of refractive error among primary school children of Malay ethnicity in Gombak district prior to the COVID-19 pandemic. A school-based retrospective data analysis was performed from January 2019 to April 2020 by random selection on Standard 1 to Standard 6 Malay students of 4 primary schools in Gombak district. Visual acuity assessment was measured using Snellen chart. Positive predictive value of uncorrected visual acuity equal or worse than 6/9, was used as a cut-off point for further evaluation by automated and retinoscopic refraction. The spherical equivalent was used with the formula (sphere plus cylinder/2). A total of 987 students were screened and evaluated. The mean subject age was 9.7 (SD \pm 1.39$)$, male represented $48.1 \%$ ( $n=$ $465)$ of subjects and female $51.9 \%(n=502)$. From the eye screening $42.2 \%$ required retinoscopic refraction. About $41.8 \%$ students had refractive errors of which $87.62 \%$ were uncorrected. Myopia is the most common type of refractive error among the students with prevalence of $52.8 \%$, followed by hyperopia $36.8 \%$ and astigmatism $10.4 \%$. The prevalence of amblyopia was $15 \%$ among the refractive group, and $71.7 \%$ of the amblyopic subjects had bilateral amblyopia. A significant positive correlation was noted between myopia development with increasing age $(P<0.005)$. Refractive error needs careful evaluation and preventive measures for the benefit of our children. The COVID-19 pandemic threatened our effort to reduce the rate of refractive error by exposing the children to the risk of myopic progression. Our study highlights an urgent need for school children to go for vision screening and needs further attention among parents and policy makers to anticipate further negative impact from it. 


\title{
POSTER PRESENTATION
}

\section{ID184 PP128}

\section{Evidence for the Anthelmintic Effect of Date Palm Fruit: From Ethno- Religious Manuscript Wisdom to Digital Imaging}

\author{
Najm, Omaima Adili1,2; Abdul-Rahman, Asma ${ }^{1}$; Addnan, Faizul Helmi ${ }^{2}$; Elkadi, Mohamed Adel ${ }^{1}$, Mohd-Manzor, \\ Nur Fariha ${ }^{1}$; Abdullah, Wan Omar ${ }^{1}$; Mansur, Fadlul Azim Fauzi ${ }^{1}$
}

${ }^{1}$ Faculty of Medicine and Health Sciences, Universiti Sains Islam Malaysia, Nilai, Malaysia

${ }^{2}$ Department of Biology, College of Education for Pure Science, Mosul University, Mosul, Iraq

${ }^{3}$ Faculty of Major Language Studies, Universiti Sains Islam Malaysia, Nilai, Malaysia

Soil-transmitted helminth infection is a global public health problem in need of alternative sources of deworming agents due to the problem of resistance to conventional chemotherapy. Referencing ethno-medical knowledge, scientists screen natural compounds from plants and animals for anthelmintic activity and found compelling anecdotal evidence in fruit enzymes, milk and tannins. We focused on referencing Islamic ethno-religious sources like the Quran, hadeeth, and scholar books for any indication of date palm fruit anthelmintic reference. With such reference, aiming to translate information into digital scientific evidence. Based upon the findings of Arabic text language expert and using the canine hookworm Ancylostoma caninum as model we investigated the anthelmintic activity of date palm fruit in vitro by describing in detail the nature of cross-sectional damage taking place in the worm cuticle after exposure to date palm fruit extract solution through careful observation of digital imaging captured through transmission electron microscopy (TEM). While general and other specific references for date palm fruit can be found in the Quran and hadeeth, no such references were found for anthelmintic effect. However, a specific prescription to kill worms by consuming date palm fruit on an empty stomach was found in the book medicine of the prophet by Ibn Jawziyya circa (1300 AD). Worm incubated in Ajwa date palm fruit extract solution exhibited cuticular damage in the form of significant thinning as well as destruction and irregularity of subcuticular architecture similarly seen with fruitderived cysteine proteinases mediated damage. Similar damage was not seen in both the positive and negative control worms. The deworming prescription using date palm fruit in the book medicine of the prophet may be substantiated by the digital evidence of worm cuticular damage. Translating manuscript based classical knowledge to digital based evidence is useful for future applications. 


\title{
POSTER PRESENTATION
}

\section{ID185 PP129}

\section{Nursing Students' Knowledge and Attitude toward Sexual Health Concerns}

\author{
Fatimah Sham ${ }^{1}$, Norhayati Abdul Rahman ${ }^{1}$, Siti Munirah Abdul Wahab², Frannelya Francis ${ }^{3}$, Norhafizatul Akma \\ Shohor $^{1}$ \\ ${ }^{1}$ Faculty of Health Sciences, Universiti Teknologi MARA Selangor Branch, Puncak Alam Campus, 42300, \\ Malaysia \\ ${ }^{2}$ Centre of Nursing, Faculty of Health Sciences, Universiti Teknologi MARA Penang Branch, 13200 Kepala Batas, \\ Penang, Malaysia \\ ${ }^{3}$ Faculty of Health Sciences, Universiti Teknologi MARA Sarawak Branch, 94300 Kota Samarahan, Sarawak, \\ Malaysia
}

Increased demand for special attention related to sexual health concerns requires expert nurses in sexual health care. Nurse education is critical in training students to fulfill the growing demand for sexual health care in the future. This research explored the nursing students' knowledge and attitude towards dealing with clients' sexual health concerns. A cross-sectional study was conducted at three different UiTM campuses. A total of 312 undergraduate and postgraduate nursing students were employed using convenience sampling approach. A self-administered questionnaire adapted from previous study. In this study, $56.1 \%$ of nursing students perceived practice is fair while $41 \%$ of them perceived knowledge is good towards sexual health assessments. Majority of them $(80 \%)$ showed positive in acknowledging the nursing role in sexual health care and found exemplars. However, less than half of them showed negative perception of client's and colleagues' response, and their own confidence. The post experience students had significant negative attitude towards their role in discussing sexual concerns $[\times 2=8.97 ; p<0.05]$ and their perception of been teased or ridiculed by their colleagues [ $x 2=11.17 ; p<0.01]$. Meanwhile, the second year and below students had significant negative attitude towards their perception of discussing sexuality would embarrass [ $x 2=11.92 ; p<0.01]$ and anxious $[x 2=11.55 ; p<0.01]$ their clients. The second year and below students had significant positive attitude towards their perception that nurses would address sexual concerns when needed $[x 2=11.29 ; p<0.01]$. Nursing students' sexual health knowledge was good; however, their assessment in practice was fair. Although the attitudes on nursing practice related to sexual health care was positive but the perception of client's and colleagues' response; and their own confidence still have negative impact on their attitudes. Considerate the learning need, defeat the barriers in improving educational programmes and clinical practice fostering the nurses for an extended role in sexual health care. 


\title{
POSTER PRESENTATION
}

\section{ID186 PP130}

\section{Cavities with intracavitary mass in COVID-19: a Diagnostic Dilemma}

\author{
Tan, Cynthia K. Y. ${ }^{1}$; Rohaizat, Fathin Izni Izzati'; Sharma, Deepali²; Ab Rahman, Siti Soraya ${ }^{3}$ \\ ${ }^{1}$ Department of Radiology, Hospital Ampang, Selangor, Malaysia \\ ${ }^{2}$ Faculty of Medicine and Health Sciences, Universiti Tunku Abdul Rahman, Selangor, Malaysia \\ ${ }^{3}$ Faculty of Medicine and Health Sciences, Universiti Sains Islam Malaysia, Negeri Sembilan, Malaysia
}

Concomitant infection in COVID-19 is not uncommon, with almost a quarter of elderly patients getting secondary bacterial infections. Co-infection with fungal, however, is rare and only several cases have been reported to date. We report a case of COVID-19 with concomitant fungal and multidrug-resistant organism (MRO) Acinetobacter baumanii infections. A 56-year-old lady tested positive for COVID-19 after she presented with fever, cough, shortness of breath and diarrhoea. Her condition rapidly deteriorated, requiring high flow nasal cannula (HFNC). Highresolution Computed Tomography (HRCT) lung performed on Day 21 of illness showed multiple cavitating lesions containing rounded mass within with surrounding air crescent, and multiple lung nodules. Commonly reported features of COVID-19 pneumonia and organising pneumonia were also present in the HRCT. Cultures of her sputum, tracheal aspirate and blood grew Acinetobacter baumanii. Antifungal treatment was immediately started. Unfortunately, she succumbed to her illness three days later. Common CT features of COVID-19 include ground glass opacities and consolidation predominantly in the lower lobes. Cavitating lung lesions are typically associated with mycobacterial, fungal, or neoplastic aetiologies. Lung cavitations have been reported following infarction and pulmonary embolism in nonCOVID-19 patients but is rarely caused by COVID-19 pneumonia. Presence of intracavitary mass and air crescent sign are highly suggestive for fungal ball. Since there is no history of pulmonary tuberculosis or preformed cavities prior to this admission, COVID-19-associated pulmonary aspergillosis is a consideration. However, mycological evidence in the form of galactomannan detection in respiratory samples or through bronchoscopy could not be obtained from this patient in view of her critical status and rapid deterioration. COVID-19 patients with superinfection and co-infection have been associated with higher mortality. If there is a high suspicion of invasive pulmonary aspergillosis, a comprehensive diagnostic workup will ensure early detection and preemptive treatment for better clinical outcome. 


\title{
POSTER PRESENTATION
}

\section{ID188 PP131}

\section{The Role of Virtually Adaptive Simulated Patients in Undergraduates Medical Training: A Scoping Review}

\author{
Zainal, Nur Zaireena ${ }^{1}$; M. Thamrin, Norashikin²; Surya Rangkuti, Harry'; Masri, Maizatul Azma ${ }^{1}$; Mohd Aris, \\ Muhammad Shamsir ${ }^{1}$ \\ ${ }^{1}$ Obstetric \& gynaecology dept; Faculty of Medicine and Health Sciences, Universiti Sains Islam Malaysia; \\ Negeri Sembilan; Malaysia \\ ${ }^{2}$ School of Electrical Engineering, College of engineering, Universiti Teknologi Mara, Shah Alam, Selangor
}

The global educational system has been seriously hampered by the growing pandemic. Due to health and safety concerns, clinical students are not permitted to enter the hospital grounds during movement restrictions. These limitations have boosted inventiveness in teaching and e-learning activities. Efforts have been made in many medical schools to achieve almost genuine clinical teaching experience as possible. Artificial intelligence has lately emerged as a promising technique for replacing clinical scenario training in clinical setting. The goal of these virtual simulated patients was to produce a standard patient to accomplish learning outcomes in history taking, diagnosis, and treatment. The purpose of this study is to examine the various forms of virtual simulated patients (VSP) technologies employed, as well as the outcomes and attitudes of students toward the methods used. ProQuest, EBSCOHOST, PubMed, Scopus, and Google Scholar were used to conduct the searches. Over the previous 20 years, English open access articles reviewed. The data was then pooled and quantitatively analyzed. Using the keywords artificial intelligence, medical education, and undergraduate students; it was found that, only 23 out of 399 articles met the inclusion criteria. In 86 percent of the discovered articles, the VSP was employed with specialized software for medical student training. Furthermore, four articles compared VSP to traditional teaching demonstrating that most students are responsive and motivated. However, there are limitations in terms of creating confidence and communicating wording that can be exploited as artificial intelligence advances. Although VSP has shown to be effective in teaching medical students communication, the real-world clinical experience cannot be replaced. 


\title{
POSTER PRESENTATION
}

\section{ID189 PP132}

\section{Highly Active Relapsing Remitting Multiple Sclerosis with Optic Neuritis and Transverse Myelitis; Fingolimod as an Induction Therapy?}

\author{
Zanirah, Fatimah'; Tan, Hui Jan'; Solehan, Hana Maizuliana'; Mohammad Kazmin, Nur Ezzaty'; Tan, Wei Ming', \\ Samad, Sarah Zulaikha ${ }^{1}$; Sharil, Nor Safiqah ${ }^{1}$; A Bakar, Mohd Firdaus ${ }^{1}$ \\ ${ }^{1}$ Department of Medicine, Faculty of Medicine and Health Sciences, Universiti Sains Islam Malaysia (USIM), \\ Nilai, Negeri Sembilan, Malaysia \\ ${ }^{2}$ Neurology Unit, Department of Medicine, Universiti Kebangsaan Malaysia Medical Centre (UKMMC), Cheras, \\ Kuala Lumpur, Malaysia.
}

Multiple sclerosis (MS) is an autoimmune demyelinating disorder with variable disease courses. Treatment is challenging, however disease-modifying therapies have shown promising outcomes. We report a case of newly diagnosed highly active relapsing-remitting multiple sclerosis (HA RRMS) in a 20-year-old female with previously undiagnosed clinical attack. She presented with sudden onset of right eye blurring of vision preceded by numbness of hands and feet, giddiness, and unsteady gait for 2 months. Right eye relative afferent pupillary defect (RAPD) was observed. Limb's examination revealed reduced power of 4/5 with absent reflexes and upgoing Babinski. There was sensory level until T6 with bilateral loss of proprioception. Cerebellar signs were positive with ataxic gait. Urgent magnetic resonance imaging (MRI) brain and whole spine revealed active demyelinating lesions at the juxtacortical, infratentorial, spinal cords, and periventricular area of the brain. Lumbar puncture showed high opening pressure $(25 \mathrm{~cm} \mathrm{H} 20)$ and high protein level $(383 \mathrm{mg} / \mathrm{L})$. She was treated as HA RRMS with optic neuritis, brainstem involvement and transverse myelitis. She completed intravenous methylprednisolone $1 \mathrm{gm}$ daily for 5 days and fingolimod was swiftly introduced. Following that, right eye RAPD resolved. Sensory and motor examinations were also improving, except hip flexion was 4+/5. The patient was treated as HA RRMS in view of her rapid clinical progression and heavy burden of brain MRI T2 lesions. Fingolimod was chosen as first line therapy to control the highly aggressive disease despite usually being the second line in the sequence of treatment. FREEDOMS trial showed that fingolimod, an immunomodulator significantly improved relapse rates and end points measured on MRI, as compared with either placebo or intramuscular interferon beta-1a in HA RRMS treatment. In a case of HA RRMS, fingolimod is utilized as part of induction therapy together with intravenous methylprednisolone to safeguard better prognosis. 


\title{
POSTER PRESENTATION
}

\section{ID190 PP133}

\section{Left Ventricular Clots with Dilated Cardiomyopathy in COVID-19 Infection; An Underreported Complication of COVID-19 Myocarditis?}

\author{
Zanirah, Fatimah'; Solehan, Hana Maizuliana ${ }^{1}$; Mohammad Kazmin, Nur Ezzaty ${ }^{1}$; Tan, Wei Ming ${ }^{1}$; Samad, \\ Sarah Zulaikha'; Sharil, Nor Safiqah'; A Bakar, Mohd Firdaus ${ }^{1}$ \\ ${ }^{1}$ Department of Medicine, Faculty of Medicine and Health Sciences, Universiti Sains Islam Malaysia (USIM), \\ Nilai, Negeri Sembilan, Malaysia.
}

Myocarditis is increasingly recognized as one of the complications of severe acute respiratory syndrome (COVID-19). Naturally, fulminant myocarditis or heart failure has also been reported in its counterpart, the Middle East Respiratory Syndrome Coronavirus (MERS-CoV). We report a case of a 34-year-old male with no known medical illness who presented with failure symptoms day 21 post-COVID-19 infection. He was treated as COVID-19 stage $4 \mathrm{~b}$ and discharged well before presenting again with shortness of breath and bilateral leg swelling for 4 days. On examination, he was tachypnoeic with raised jugular venous pressure. Apex beat was displaced with bibasal fine crepitations and leg swelling. Chest radiograph showed cardiomegaly with bilateral pleural effusions and electrocardiogram showed sinus tachycardia with poor R-wave progression. An echocardiogram revealed dilated chambers with global hypokinesia. Left ventricular clots of varying sizes were seen with ejection fraction of (25-30) \%. Warfarin was commenced, his fluid intake was restricted, and anti-failure medications were optimized. Patient was discharged well and referred to Serdang Hospital Cardiologist for further management. Myocarditis in COVID-19 occur due to direct viral injury or from exaggerated host immune response. The diagnosis is established based on detail history, clinical examination, laboratory findings and non-invasive imaging studies. Fever, shortness of breath, cough, and chest pain were the most common reported symptoms. Electrocardiogram changes include non-specific ST-segment and T-wave changes and ventricular tachycardia. Most patients had left ventricular failure with elevated cardiac and inflammatory biomarkers. Cardiac magnetic resonance imaging is the preferred imaging modality when available and endomyocardial biopsy may be performed if the diagnosis remains uncertain. COVID-19 myocarditis should be considered as one of the differential diagnoses in COVID-19 patients with failure symptoms, particularly young patients with previously unknown medical illness as it could lead to significant morbidity and mortality. 


\section{POSTER PRESENTATION}

\section{ID195 PP134}

\section{Macklin Phenomenon as a Complication of COVID-19}

Samad, Sarah Zulaikha',2; Sia, Koon Ket ${ }^{2}$; Solehan, Hana Maizuliana ${ }^{1}$; Tan, Wei Ming ${ }^{1,2}$; Mohammad Kazmin, Nur Ezzaty ${ }^{1}$; Zanirah, Fatimah ${ }^{1}$

${ }^{1}$ Department of Medicine, Faculty of Medicine and Health Sciences, Universiti Sains Islam Malaysia (USIM), Nilai, Negeri Sembilan, Malaysia

${ }^{2}$ Department of Medicine, Hospital Ampang, Selangor, Malaysia.

Spontaneous pneumothorax and spontaneous pneumomediastinum has been reported in literature as a complication of COVID-19, even in patients who have never been mechanically ventilated. Diagnosis can be challenging, especially if the clinician is not vigilantly looking out for it. Patients can be disproportionately ill, requiring higher oxygen requirement than what is expected. We report a case of a COVID-19 positive 25-year-old primigravida who presented to our hospital Patient Assessment Centre (PAC) with reduced fetal movements, fever, and cough. At the PAC, the cardiotocography (CTG) showed fetal tachycardia, and the obstetrics team proceeded with emergence lower segment caesarean section (LSCS) for suspected chorioamnionitis, via spinal anaesthesia. Post-delivery she was transferred to the obstetric ward, and she was initially comfortable on room air. She desaturated on day 2 of admission and required $15 \mathrm{~L}$ high flow mask oxygen. She was treated as presumed cytokine release syndrome and pneumonia as her chest-x-ray worsened, and rising C-reactive protein and white cell count. She was started on steroids and antibiotics. A CT Pulmonary angiography done on day 8 of admission showed extensive pneumomediastinum and minimal pneumothorax. Discussion with cardiothoracic team agreed for a conservative management. Patient was discharged well after 2 weeks of admission once she was able to be weaned off oxygen. Pneumomediastinum in COVID-19 is the result of Macklin phenomenon where the alveolar air which is released from alveolar rupture tracks along peribronchial vascular sheaths towards the mediastinum. Treatment is mostly supportive unless presence of haemodynamic instability or difficult ventilation. Pneumothorax and pneumomediastinum are possible complications in COVID and the clinician should always be aware of its possibility especially if patient's respiratory symptoms is disproportionate to the severity of the chest $x$ ray as well as the inflammatory parameters for example total white count and CRP. 


\section{POSTER PRESENTATION}

\section{ID196 PP135}

\section{Digital Health Literacy and Online Information Seeking during COVID- 19: Are We Digitally Literate?} $\frac{\text { Khebir, Norliyana A }}{\text { Liyana }^{2}}$; Mohd R, Nur SA ${ }^{1}$; Mohmad L, Nur FA ${ }^{1}$; Nik MA, Nik HAJ'1 ; Azzeri, Amirah ${ }^{1}$; Azmi,

${ }^{1}$ Public Health Unit, Department of Primary Health Care, Faculty of Medicine and Health Sciences, Universiti Sains Islam Malaysia

${ }^{2}$ Department of Microbiology, Faculty of Medicine and Health Sciences, Universiti Sains Islam Malaysia, 71800, Nilai, Negeri Sembilan

The World Health Organization (WHO) defines digital health literacy as the ability to seek, find, understand, and appraise health information from electronic sources and apply the knowledge gained to addressing or solving a health problem. The current pandemic has accelerated the paradigm of digital health worldwide. Developing countries are facing significant problems to adopt digital health due to low digital health literacy (DHL). This review aims to describe the DHL and its associated factors among university students in Malaysia. A cross-sectional study among university students from various categories was conducted. The data collection was conducted through an online survey via Google form through various social media. The questions asked were adopted from a validated questionnaire. Following descriptive analysis, bivariate analysis was conducted accordingly. 202 university students participated in this study. Most of the participants were female, Malay, undergraduate students and in the M40 family. $80 \%$ of the participants reported that when they search for information on the COVID-19 it was easy for them to find information. $71 \%$ of them believe that when they share information about the COVID-19 on social media, it was easy for them to formulate and express their questions, opinions, and thoughts. $90 \%$ of them revealed that they obtained reliable information and they can apply the knowledge in their daily life. More than half of them believe that online information seeking is safe. The bivariate analysis found that the level of digital health literacy was higher among those in 18-23 years old, female and those in T20 families ( $p$-value $<0.05$ ). This study found that the digital health literacy and online information seeking among university students in Malaysia was high. Having a foundation on computer and Internet enable them to use and apply digital health easily. It is also useful for them to get information on the COVID19 pandemic. 


\section{POSTER PRESENTATION}

\section{ID198 PP136}

\section{Advancement of Telemedicine in Southeast Asia During COVID-19 Pandemic: A Review}

Fauzi, Shyafiqah ${ }^{1}$; Azmi, Liyana ${ }^{2}$

${ }^{1}$ Faculty of Medicine and Health Sciences, Universiti Sains Islam Malaysia, 71800, Nilai, Negeri Sembilan

${ }^{2}$ Department of Microbiology, Faculty of Medicine and Health Sciences, Universiti Sains Islam Malaysia, 71800, Nilai, Negeri Sembilan

The transmission of COVID-19 infection has increased risks of contracting the disease particularly for patients in primary care centers and outpatients. To reduce the risks of infection, telemedicine can minimize exposure to COVID-19 risk infection. Telemedicine is an efficient and cost-effective way to deliver healthcare services by using information and communications technology (ICT). This review studies the progress of telemedicine services in the Southeast Asia (SEA) region during COVID19 pandemic, focusing on specific type of healthcare services and patients using telemedicine. The methodology employed in this research were the combination of relevant article searched through Google Scholars and Research Gate by using keywords: "Telemedicine", "COVID-19", "Southeast Asia", "Malaysia”, "Indonesia”, "Singapore", "Thailand", "Vietnam", "Brunei”, "Vietnam", "Myanmar", "Cambodia" and "Timor Leste". Telemedicine contributes 36.36\% of SEA's health deliveries from Indonesia, Malaysia, Singapore, and Vietnam focusing on various specialties and subspecialties of internal medicine as an alternative to usual clinic visits, preventing overcrowding and long queue patients. In Myanmar, face-to-face consultation combined with telemedicine is used for easier follow ups. Teleconsultation in Indonesia as Tele-ECG and Vietnam in cardiovascular surgery program. $18.18 \%$ countries in this region namely Vietnam and Thailand also implement telemedicine as a tool for examination, diagnostic and monitoring purposes. Telerehabilitation services in the Philippines emphasize rehabilitation consultation and follow-up. Teleprescribing in Singapore requires the physician's approval for medication which is done by email. For Brunei, Timor Leste, Cambodia, and Laos there were no reported telemedicine services during COVID-19 pandemic. COVID-19 pandemic has accelerated the need for implementation of telemedicine in healthcare systems in Southeast Asia. The use of telemedicine is required to reduce the spread of the disease, protect both patients and staff and improve clinic visits restricted by movement control order. 


\section{POSTER PRESENTATION}

\section{ID200 PP137}

\section{Gene Expression as a Precision Medicine Tools in Cervical Cancer: A Review}

Abdul Hamid, Nazefah ${ }^{1}$; Roslan, Nuruliza ${ }^{1}$; Zainal, Nur Zaireena2² Abd Rahman, Hayati ${ }^{3}$

${ }^{1}$ Department of Basic Medical Sciences I, Faculty of Medicine and Health Sciences, Universiti Sains Islam Malaysia, Persiaran Ilmu, Putra Nilai, 71800, Nilai, N. Sembilan, Malaysia

${ }^{2}$ Department of Surgical-Based, Faculty of Medicine and Health Sciences, Universiti Sains Islam Malaysia, Persiaran Ilmu, Putra Nilai, 71800, Nilai, N. Sembilan, Malaysia

${ }^{3}$ Department of Basic Medical Sciences II, Faculty of Medicine and Health Sciences, Universiti Sains Islam Malaysia, Persiaran Ilmu, Putra Nilai, 71800, Nilai, N. Sembilan, Malaysia

Cervical cancer is one of the leading causes of cancer-related deaths in women worldwide. Cervical cancer is caused by sexually acquired infection of the Human Papillomavirus (HPV) with high-risk types 16 and 18 that causes $70 \%$ of cervical cancer and pre-cancerous cervical lesions. The use of molecular techniques in precision medicine has been widely utilised as a means towards diagnostic and prognostic purposes. This study aims to review a detailed description of gene expression as biomarkers for precision medicine specifically in cervical cancer research. A literature review search was conducted using online databases including PubMed, Scopus and ScienceDirect. The searched keywords were cervical cancer AND personalised medicine. All articles in English including review and experimental articles were then reviewed and analysed accordingly. It has been reported that Next Generation Sequencing (NGS), microarray and single nucleotide polymorphism (SNP) are some of the molecular techniques used to determine biomarkers in cervical cancer that can possibly be used as a precision medicine tool. Interleukin-6 rs1800795 polymorphism, apoptotic genes, hypoxia-inducible genes, tumor cell invasion and metastasis genes were reportedly found to be increased in cervical cancer. Expression of these genes are influenced by the genetic and epigenetic factors, for example aberrant miRNA expression and changes in DNA methylation status. These genes are predicted to be novel biomarkers for the detection of cervical cancer, thus increasing its potential to be used in precision medicine in cervical cancer. In the era of precision medicine, novel biomarkers have proven to be useful for screening, testing and treatment of diseases. These may play an important role in the prediction of early recurrence and can lead to alternative treatment options. 